\title{
MODULATING VERBAL EPISODIC MEMORY ENCODING WITH TRANSCRANIAL ELECTRICAL STIMULATION
}

\author{
Dissertation \\ for the award of the degree \\ "Doctor of Philosophy" (PhD) \\ Division of Mathematics and Natural Sciences \\ of the Georg-August-Universität Göttingen \\ within the doctoral program "Biology" \\ of the Georg-August University School of Science (GAUSS)
}

submitted by Gabriel Amador de Lara

from Tubarão, Brazil

Göttingen, 2018 


\section{Thesis Committee}

Prof. Dr. Walter Paulus (Supervisor, First Referee)

Georg-August-Universität Göttingen

Klinik für klinische Neurophysiologie

Universitätsmedizin Göttingen

Robert-Koch-Straße 40, 37075 Göttingen

Prof. Dr. Lars Penke (Second Referee)

Georg-August-Universität Göttingen

Georg-Elias-Müller-Institut für Psychologie

Abteilung Biologische Persönlichskeitpsychologie

Goßlerstraße 14, 37073 Göttingen

\section{Members of the Examination Board:}

\section{PD Dr. Peter Dechent}

Georg-August-Universität Göttingen

Abteilung Kognitive Neurologie

Universitätsmedizin Göttingen

Robert-Koch-Str. 40, 37075 Göttingen

\section{Dr. Roberto Goya-Maldonado}

Georg-August-Universität Göttingen

Systems Neuroscience and Imaging in Psychiatry Lab

Universitätsmedizin Göttingen

Von-Siebold-Straße 5, 37075 Göttingen

\section{Prof. Dr. Susan Boretius}

Georg-August-Universität Göttingen

Functional Imaging Laboratory

German Primate Center

Kellnerweg 4, 37077 Göttingen

Prof. Dr. Hansjörg Scherberger

Georg-August-Universität Göttingen

Deutsches Primatenzentrum

Abteilung Neurobiologie

Kellnerweg 4, 37077 Göttingen

Date of oral examination: 22.10.2018 


\section{Statement of Originality}

I hereby declare that this thesis has been written independently with no other sources and aids that quoted in the text, references and acknowledgments.

Göttingen, 07.09.2018

Gabriel Amador de Lara 
vi la circulación de mi oscura sangre, vi el engranaje del amor y la modificación de la muerte, vi el Aleph, desde todos los puntos, vi en el Aleph la tierra, y en la tierra otra vez el Aleph y en el Aleph la tierra, vi mi cara y mis vísceras, vi tu cara, y sentí vértigo y lloré, porque mis ojos habían visto ese objeto secreto y conjetural, cuyo nombre usurpan los hombres, pero que ningún hombre ha mirado: el inconcebible universo.

..."

Jorge Luis Borges, El Aleph

Abriu-se majestosa e circunspecta, sem emitir um som que fosse impuro nem um clarão maior que o tolerável

pelas pupilas gastas na inspeção contínua e dolorosa do deserto, e pela mente exausta de mentar

toda uma realidade que transcende a própria imagem sua debuxada no rosto do mistério, nos abismos.

Abriu-se em calma pura, e convidando quantos sentidos e intuições restavam a quem de os ter usado os já perdera

e nem desejaria recobrá-los, se em vão e para sempre repetimos os mesmos sem roteiro tristes périplos,

convidando-os a todos, em coorte, a se aplicarem sobre o pasto inédito da natureza mítica das coisas, 


\section{Table of Contents}

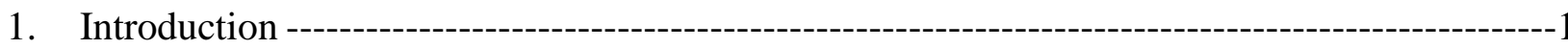

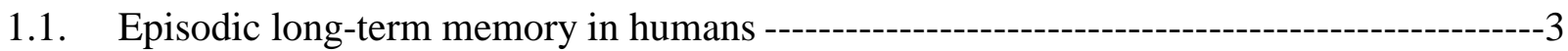

1.2. Transcranial electrical stimulation (tES) ---------------------------------------------------------6

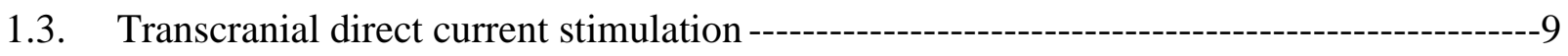

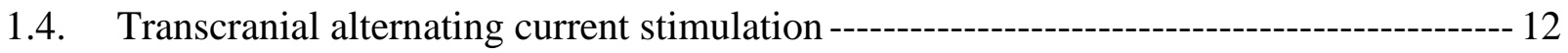

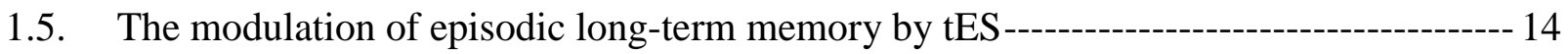

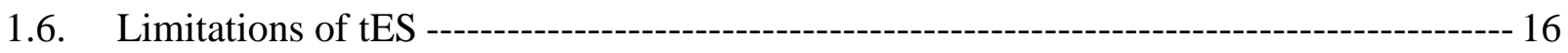

2. Targeting the left pre-frontal cortex during verbal episodic memory encoding with transcranial direct current stimulation ------------------------------------------------------------------------------------- 18

3. Targeting the left temporal cortex during verbal episodic memory encoding with transcranial

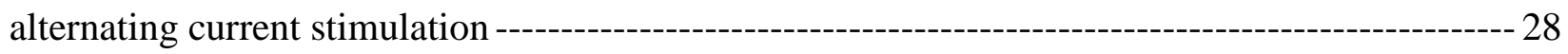

3.1. Supplementary material ---------------------------------------------------------------------------- 61

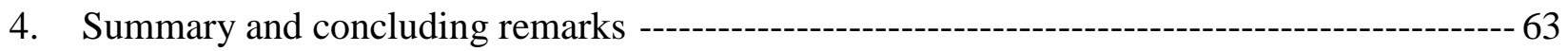

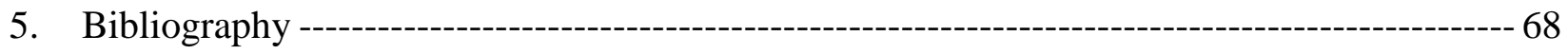

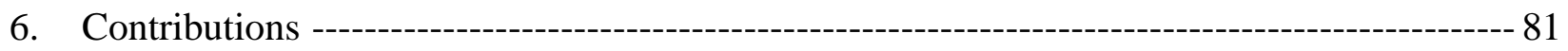

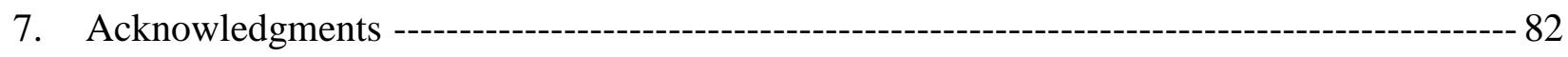

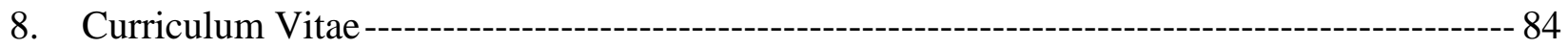




\section{Introduction}

Understanding and explaining behavior has been an ongoing labor and permanent quest for the human kind. From metaphysical speculations to the first elaborations of this method of manipulating and decomposing perceptual information nominated science, explaining and predicting living organisms' behaviors was a first-order agenda. The outbreak of modern neuroscience and psychology approaches allowed direct experimentation with the mind-brainbehavior conundrum, leaning on an extensive row that goes from lesioned patients to modern neuroimaging methods. The in-depth study of the physiological foundations of higher brain functions and mental activity in the mammalian brain is denominated cognitive neuroscience, where cognitive processes that rely on the central nervous system, such as memory, can be scientifically investigated with advanced methods such as imaging and computational techniques (Raichle, 2009).

Some of the modern neuroimaging techniques, as electrophysiology and magnetic resonance tomography, have paved the way to unveil the neural basis of cognition and behavior, each with its own qualities and pitfalls, such as their temporal and spatial resolution (Axmacher, Elger, \& Fell, 2009). In conjunction with them, non-invasive brain stimulation (NIBS) are techniques widely employed in the last decades to the study of physiology of cognitive processes, and bring the promise of being a complementary therapeutic intervention to neuropsychiatric disorders (Parkin, Ekhtiari, \& Walsh, 2015). They can be useful to causally connect certain brain structures and neuronal activity to functions, since neuroimaging and electrophysiology can reveal spatio-temporal fingerprints of cognitive processes, but can only establish a correlational bound between them (Bergmann, Karabanov, Hartwigsen, Thielscher, \& Siebner, 2016). On the other 
hand, NIBS can be inserted as a perturbation to the brain in order to interfere with a targeted function. In the scope of memory research, NIBS, specifically transcranial Electrical Sitmulation (tES) interventions, have been applied to several brain areas with the use of direct and alternating currents. In an atempt to modulate memory encoding or retrieval, the majority of the studies focused on the pre-frontal cortex as a preferable target (Manenti, Cotelli, Robertson, \& Miniussi, 2012).

In this doctoral dissertation, we attempted to advance in methodologically rigorous protocols influencing normal brain physiology and search for solutions to modulate memory activity, a promising area for rehabilitation of people with neuropsychiatric diseases or aging impairments. The episodic memory network is composed of several brain nodes working in a network manner. For that, we attempted to influence different nodes of this network (here the left pre-frontal and temporal cortices) in the search of the most robust and convincing results. We made use of different versions of a verbal-associative memory task as the behavioral paradigm, and computational models to estimate the features of our stimulation parameters. Specifically, the aim of this doctoral work was to augment long-term memory performance in healthy human participants by the application of transcranial electrical stimulation. In the following pages, I outline the state of the art of the methods and brain processes investigated in this work, before introducing the original scientific contributions brought by the two manuscripts that form the core of this thesis. The last chapter summarizes the results brought by our investigations and points possible future directions for the field. 


\subsection{Episodic long-term memory in humans}

The brain systems supporting learning and memory are classified and differ according to type and functional relevance. Short-term and long-term memory are partially overlapping functions which rely on distinct neural underpinnings, being the long-term system split into procedural (or implicit) and declarative memory (Morris, 2013). Episodic long-term memory is the process of learning, storing and retrieving information in the brain regarding to daily personal experiences, and a crucial feature for humans to thrive and adapt in different ever-changing environmental conditions (Tonegawa, Pignatelli, Roy, \& Ryan, 2015). While in the short-term modality memories seem to be held in patterns of neural activity, long-term encompass structural modifications of synaptic linkages and network connectivity. The encoding of a long-term memory involves an initial scheme of activity that promotes a posterior structural modification to be retained as a trace, i.e., an arrangement of connections maintained in specific neural assemblies by structural molecular changes (Morris, 2013). The intriguing kaleidoscope of long-term memory representations in the brain (namely the "engram") are ensued by epigenetic modification in gene expression caused by cascades of molecular alterations in neural activity to sensory inputs (Poo et al., 2016). These molecular modifications, such as increase in AMPA receptors and spines that control post-synaptic gears, are promoted and altered depending on the regularity of the activity between cell assemblies that regulate the firing of a postsynaptic neuron, fostering the core mechanisms of synaptic plasticity, the so-called long-term potentiation (LTP) or depression (LTD). In mammals, the molecular and cellular basis of episodic memory encoding can be approached by invasive procedures and cellular recordings. In humans, such invasive recordings 
are poorly available, with the mechanisms of long-term memory having to be uncovered mostly by techniques such as electrophysiology and neuroimaging (Kandel, Dudai, \& Mayford, 2014).

A large body of literature has argued that declarative long-term memory is implemented on intertwined distributed networks paced in spatiotemporal scales. Neocortical, allocortical and subcortical brain areas were already extensively studied with the help of lesioned patients, neuropsychological and neuroimaging findings. The most common areas associated with episodic long-term memory are regions in the pre-frontal $(\mathrm{PF})$, parietal cortex $(\mathrm{PC})$ and the temporal lobe, especially its medial part, with feedback projections between the neocortical associational, subcortical and medial temporal lobe (MTL) structures (Dickerson \& Eichenbaum, 2010; Henke, 2010; Kühn \& Gallinat, 2014; Ofen et al., 2007; Preston \& Eichenbaum, 2013).

The last decades have seen the rise of a method to compare neural activity regarding patterns of activation during recall or forgetting on memory tasks, called Subsequent Memory Effect (SME), a biomarker for either successful encoding or for neural activity interfering with it (Kandel et al., 2014). A meta-analysis investigated 74 fMRI episodic memory studies during encoding of item and associated verbal and pictorial content, and showed a SME associated mainly with the left inferior frontal cortex, bilateral fusiform cortex, bilateral medial temporal lobe, bilateral premotor cortex and bilateral posterior parietal cortex (H. Kim, 2011). The verbal associative memory modality (explored in this thesis) predominantly presented left-lateralized SM effects. These results in the verbal associative domain were partially confirmed by a later fMRI study, with effects being left-lateralized at the pre-frontal cortex level, but bilateral for the medial temporal lobe and posterior parietal cortex (Schott et al., 2013). Gilmore et al. (2015) expounded on a group of metaanalyses of task based functional MRI studies, proposing a functional parietal memory network that showed SME for encoding and successful retrieval, encompassing the precuneus, the mid- 
cingulate cortex, and the posterior inferior parietal lobule. In turn, the functional role of the MTL on episodic memory is well stablished, with extensive scientific evidence showing a functionrelated topography in its different structures operating in a loop-coordinated fashion. The hippocampus is strongly associated with relational memory, i.e. for storing and binding the different associative memory representational elements (Davachi, 2006; Hannula \& Ranganath, 2009; Henke, 2010; Jeong, Chung, \& Kim, 2015; Ranganath, 2010).

Neural oscillations are deemed to temporally pace neural firing and shape plasticity by the synchronization or desynchronization of neuronal assemblies (Hanslmayr, Staresina, \& Bowman, 2016). Neuronal synchronization represents the dynamic activation of neuronal groups and underlies effective cell communication (Wang, 2010; Womelsdorf et al., 2007). The rhythmic modulation of synaptic communication is thought to be at the core of effective connectivity, and coherence between pre and post synaptic groups is required to coordinate input arrival at precise excitability phases in oscillatory cycles (Fries, 2015). In long-term memory encoding, these patterns are represented by activity in the key areas spreading along some of the canonical frequency bands. Activity correlated with successful long-term memory formation showed increases or decreases depending on the task type and site. Nevertheless, in general, decreases in alpha and beta band (desynchronization), increases in theta and gamma power (synchronization) and cross-frequency relationships are the most prominent (Düzel, Penny, \& Burgess, 2010; Hanslmayr et al., 2016; Hanslmayr \& Staudigl, 2014; Jutras \& Buffalo, 2010). In brain physiology, cross-frequency coupling (CFC) is a multiscale neural phenomenon that can be characterized by excitability fluctuations represented by the phases of the slower oscillation, which, in turn, can selectively entrain higher-frequencies spiking of local specific networks in a phase-locked manner (Canolty \& Knight, 2010; Hyafil, Giraud, Fontolan, \& Gutkin, 2015). One 
of the most distinctive observable cross-frequency phenomenon in the brain is the theta-gamma phase-amplitude coupling. It is hypothesized to be active in mnemonic computations in several neocortical and medial temporal lobe areas with a specific functional significance: theta cycles would organize distinct timing phases of neural excitability, coordinating the firing of local neural assemblies (gamma activity) that represent and segregate chunks of information (Colgin, 2015; Heusser, Poeppel, Ezzyat, \& Davachi, 2016; Lisman \& Jensen, 2013). Nevertheless, studies investigating theta-gamma phase-amplitude coupling in verbal long-term memory encoding are correlative, and the requirement of increases or decreases in theta-gamma coupling for successful encoding in humans is still under debate (Lega, Burke, Jacobs, \& Kahana, 2016; Vaz, Yaffe, Wittig, Inati, \& Zaghloul, 2017) .

The widespread nature of the episodic long-term memory network, and its multiscale requirements for effective encoding of information nurture the necessity of further unveiling its intricate anatomical and physiological underpinnings. Some of its core features, such as integration of segregated sensorial input, dispersion and division of tasks - i.e. attention allocation and local computations - and induction of effective plasticity, can be approached with neuroscientific tools able to induce plastic after-effects and/or act on the temporal scales of cognitive phenomena. For that, transcranial electrical stimulation in its modern format comes to hand, and its characteristics will be described in the next sections.

\subsection{Transcranial electrical stimulation (tES)}

Transcranial electrical stimulation is a NIBS technique that grew steadily in the last 20

years, although having an older history of human attempts on its usage. Since the convention of 
the voltaic pile, scientists have been trying to apply direct current to the human head to a myriad of neurological and behavioral conditions (Paulus, 2011). Soon these efforts were interrupted by the lack of reliable results and experimental outcomes that could be precisely measured by the time's instrumentation. The field experimented a renaissance around two decades ago, when transcranial magnetic stimulation (TMS) was used to assess motor cortex excitability after the application of direct currents (Priori, Berardelli, Rona, Accornero, \& Manfredi, 1998). Pioneer studies on direct current applied to the primary motor cortex (Nitsche \& Paulus, 2000, 2001) set up the stage for a steep increase of papers in the field, going from motor to perception, cognition and clinical studies.

tES basically assumed three different forms in the last decade: direct current (tDCS), alternating current (tACS) and random noise (tRNS), which are mostly used to interfere or enhance neuronal activity (Bergmann et al., 2016). It works through the application of weak electric currents to the scalp, generating an electric field and related electric currents in the head (Peterchev et al., 2012). In contrast to TMS, which as a suprathreshold stimulation is able to elicit neuronal firing, tES is a subthreshold form of NIBS that is capable of inducing changes in the margin of a neuronal response and up or down-regulate neuronal excitability (Fertonani \& Miniussi, 2017). The distribution of the generated-electric fields is heavily dependent on several variables, such as the experimental parameters and the individual anatomy of the target (Opitz, Paulus, Will, \& Thielscher, 2015). The anatomical compartments' conductivity values play an important role, being unique for skin, scalp, corticospinal fluid, bone, grey and white matter, given that the currents have to surpass several of them (Opitz, Windhoff, Heidemann, Turner, \& Thielscher, 2011). Gyral geometry is also crucial, since the polarization of neurons is directly related to the 
direction of the electric field and to which extent and direction it reaches different compartments of grey matter neurons (Thielscher, Opitz, \& Windhoff, 2011).

The tES technique encompasses the application of low intensity currents ( 1-4 mA) by dedicated stimulators with different assemblies of electrodes over the scalp. Electrodes vary in size (usually from 1 to 7 centimeters) according to stimulation design, and are made of rubber or plastic, normally using paste, gels or saline solution as conductive means (Fertonani \& Miniussi, 2017). Montage choices to target a selected brain area are supposed to be guided by the available literature or experimental data, as well as the use of recently available tools to simulate the generated-electric field direction and distribution, either to serve research or clinical purposes (Bergmann et al., 2016). Some key variables that impact tES are electrode type and size, conductive mean, stimulation intensity, polarity, duration, frequency and blinding protocols, all selected carefully to assure maximal protocol effectiveness and safety (Woods et al., 2016). The approach can be either online or offline, i.e., in offline, tES is applied before a task or brain monitoring in order to prior modulate brain plasticity and/or activity, whereas in online tES is delivered during a specific task and/or brain monitoring in order to direct influence behavior or brain activity during a chosen measurement. Although these parameters are controllable, several factors related to individual differences, such as brain anatomy and dynamics, neurochemistry, genetics, age and gender are hard to control and impact directly on the physiological responses to tES (Li, Uehara, \& Hanakawa, 2015).

The experimental validations of the mechanistic hypothesis underlying the action of tES method has been built up through in vitro, in vivo, computational and pharmacological studies. A pertinent discussion to the field is referred to the translation of these studies to humans, i.e., if the weak electrical currents would penetrate the scalp and actually reach the human brain, and to which 
extent of the electric filed distribution. Despite that realistic finite-element models showed that most of the current is shunted by the skin (Holdefer, Sadleir, \& Russell, 2006), recently available intracranial recordings in humans demonstrated that the currents can reach up to $0.5 \mathrm{mV} / \mathrm{mm}$ with a $1 \mathrm{~mA}$ stimulation, and, in the case of alternating currents, only negligible phase shifts across space occur (Y. Huang et al., 2017; Opitz, Falchier, Yan, Yeagle, \& Linn, 2016).

Regarding safety, ethical and legal parameters, recent literature based on more than 8.000 participants summarized the actual safety indicators of low intensity tES. So far, the majority of adverse effects were mild, such as itching or burning sensations and headache, with very occasional moderate adverse effects related to skin burning (A. Antal et al., 2017). The almost two decades of current-tES parameters in humans, in addition to the safety analysis drawn by animal studies (Liebetanz et al., 2009), have been demonstrating the feasibility of this techniques as an easy, portable and relatively safe procedure to modulate brain activity for basic and applied purposes in a tolerable fashion.

\subsection{Transcranial direct current stimulation}

Transcranial direct current stimulation is a NIBS technique that works through the application of weak direct currents over the scalp. It has the advantage of being reasonably cheap, portable and of easy applicability, making it accessible to several research and clinical facilities. Besides it's common usage with neuromodulatory purposes to cognitive, perceptual and motor processes, it has being trialed, with relative success, to a myriad of neuropsychiatric disorders, such as stroke, Parkinson, depression and chronic pain (A. Antal et al., 2017; Lefaucheur et al., 2017). 
In tDCS, the current is unidirectional and passed by a pair or set of electrodes with a determined electrode polarity, the so-called anodal or cathodal tDCS. This current direction is thought to induce long-lasting polarity-dependent effects on neuronal excitability by modulating ongoing neuronal activity, being highly dependent on the network state of the target (Andrea Antal, Terney, Poreisz, \& Paulus, 2007; Nitsche et al., 2008; Woods et al., 2016). The current is able to cross the initial anatomical compartments and affect relative wide cortical areas, initially de- or hyperpolarizing resting membrane potentials, but, depending on the duration of the stimulation, induce synaptic plasticity, and thus, long-lasting effects (Bikson et al., 2018; Polanía, Nitsche, \& Ruff, 2018). Nonetheless, it is known that the large cortical folding and the geometry and placement of the neurons in the cortical layers impact closely the quantity of polarization, where the orientation of the neurons relative to the electric field, as well as to which neuronal compartment these fields are reaching, largely determine possible net summation effects that can result in increased or decreased excitability (Thomas Radman, Ramos, Brumberg, \& Bikson, 2009; Rahman et al., 2013).

The effects of tDCS can be split into on-line and off-line. During stimulation (on-line effects), pharmacological and TMS studies revealed that changes in the resting membrane potential are likely the main actor, with no prominent effects of synaptic plasticity (Stagg \& Nitsche, 2011). Early evidence of DC fields applied to in vitro preparations showed an increase or decrease in the firing rate of neurons with the application of anodal and cathodal currents, respectively (Stagg, Antal, \& Nitsche, 2018), confirmed by recent studies that were able to manipulate membrane potential and influence up- or down-regulation of neuronal compartments excitability (Bikson et al., 2004; Thomas Radman et al., 2009). The effects of tDCS on synaptic plasticity seem to be CA2+ and NMDA receptor dependent and alter GABAergic and Glutamatergic activity, as well 
as brain neurotrophic factor-related, thus resembling LTP and LTD-like plasticity (Y.-Z. Huang et al., 2017; Nitsche, Müller-Dahlhaus, Paulus, \& Ziemann, 2012; Nitsche \& Paulus, 2011). In addition, as suggested by several pharmacological studies, the off-line effects of tDCS seem to rely on neuromodulators as dopamine, acetylcholine and serotonin for the anodal polarity, and on changes of glutamatergic synapses for the cathodal stimulation (Kuo, Grosch, Fregni, Paulus, \& Nitsche, 2007; Kuo, Paulus, \& Nitsche, 2008; Nitsche et al., 2006, 2009).

tDCS is also able to modulate brain connectivity and neural communication (To, De Ridder, Hart Jr., \& Vanneste, 2018). Studies in resting-state fMRI demonstrated the ability of tDCS to alter functional connectivity of the motor cortex, pre-frontal cortex, fronto-parietal and defaultmode networks (Amadi, Ilie, Johansen-Berg, \& Stagg, 2014; Keeser et al., 2011; Sehm et al., 2012), which can be a source of explanation to the behavioral effects resulting of its application verified in diverse experimental results. Moreover, as physiological brain oscillations are deemed to functionally underlie neural communication and synchronization, influencing them should bring detectable physiological and behavioral impacts. Direct current was demonstrated to influence ongoing oscillatory activity in several bands (theta, alpha, beta and gamma), however, without a clear mechanistical explanation so far (Notturno, Marzetti, Pizzella, Uncini, \& Zappasodi, 2014; Soekadar et al., 2013; Zaehle, Sandmann, Thorne, Jäncke, \& Herrmann, 2011). Recent intracranial recordings in monkeys during a learning task showed that tDCS is able to modulate neural excitability and connectivity, specifically tuning up connectivity in higher frequencies (Krause et al., 2017). In this doctoral thesis, tDCS is used in one study in an attempt to modulate cortical activity at the pre-frontal cortex during a verbal memory task. 


\subsection{Transcranial alternating current stimulation}

To dynamically coordinate neural transmission between functionally connected but spatially distributed brain networks, neural oscillations are claimed to play a decisive role. These oscillations represent a rhythmic activity in neuronal excitability in which components like frequency, phase and amplitude are modulated through specific functions to be performed by the brain (Thut, Miniussi, \& Gross, 2012). The poor temporal resolution of tDCS brought the field of tES research to experiment with oscillating currents. Transcranial alternating current stimulation shares common features with tDCS, such as the dependence on parameters as intensity, current density, electrodes choice and target site, but brings into play new variables to be explored, mainly frequency and phase. Frequencies from close do DC up to the kilohertz range can be employed by applying currents in an oscillatory fashion, and with that, endogenous activity can be coupled by the means entrainment, i.e., inducing changes by an external driving force and, in this case, modulating neuronal firing synchronicity that rise as detectable brain rhythms (Andrea Antal et al., 2008; Andrea Antal \& Herrmann, 2016; Miniussi, Harris, \& Ruzzoli, 2013). On one side, tACS can be a powerful tool to causally link brain oscillations to functions (Herrmann, Rach, Neuling, \& Strüber, 2013). On the other side, since several brain disorders are related to perturbations in rhythmic oscillatory patterns in cortical and subcortical areas (so-called oscillopathies), tACS turns to be a promising intervention to treat symptoms of central nervous system diseases (Fröhlich, Sellers, \& Cordle, 2015).

One of the main hypotheses behind tACS action, coming from in vitro and in vivo studies, is that the generated fields can tune up or down oscillatory power by synchronizing or desynchronizing neuronal networks. It would change spike rate and timing, both magnified by 
networks dynamics (Davide Reato, Rahman, Bikson, \& Parra, 2013). In in vitro preparations, oscillatory currents can also accomplish membrane polarization, although within the temporal characteristics of alternating fields (Deans, Powell, \& Jefferys, 2007; T. Radman, Su, An, Parra, \& Bikson, 2007). Data from ferrets' cortical slices showed that AC fields from $0.5 \mathrm{mV} / \mathrm{mm}$ could already modulate ongoing neural functioning (Fröhlich \& McCormick, 2010). A computational model, tested at the in vitro level, found that weak AC currents can affect firing time of neurons (which summed at the network level), adjust the timing of frequency cycles and pace timing shifts (D. Reato, Rahman, Bikson, \& Parra, 2010). Another computational model tested in vivo (multiunit activity recordings) in anesthetized ferrets showed an increase in oscillatory power and phaselocked activity at the frequency of the stimulation (Ali, Sellers, \& Frohlich, 2013). Recording invasively in rats, intrinsic slow oscillatory activity could be entrained by external AC fields in widespread cortical sites, where additional intracellular recordings showed the pacing of firing activity by the applied electrical current (Ozen et al., 2010).

The main mechanistic hypothesis underlying the action of tACS in the brain is that it acts by the means of entrainment (Fröhlich \& Schmidt, 2013; Helfrich et al., 2014). Entrainment corresponds, in this case, to the possibility of resetting, by an acting external force, network activity driven by local dynamics. This would phase-lock and synchronize brain oscillations in its harmonics and sub-harmonics frequencies, mostly or more strongly when the external field matches the ongoing oscillatory dominant frequency of the targeted neuronal group (Andrea Antal \& Herrmann, 2016; Miniussi, Brignani, \& Pellicciari, 2012; Veniero, Vossen, Gross, \& Thut, 2015). If tACS is able to entrain naturally occurring brain oscillations, this may signify on the neuronal level that the current modulation of the firing rates of neurons will force a temporal oscillatory shift on membrane potential, possibly influencing physiological neural rhythms (Thut, 
Schyns, \& Gross, 2011). If brain oscillations are a force behind neural communication and cognitive processes, affecting these rhythms would be detectable by behavioral changes in response to perceptual or cognitive tasks. There are currently several electrophysiological and behavioral evidences in favor of the entrainment hypothesis in tACS (Romei, Thut, \& Silvanto, 2016). Long-lasting effects of tACS are scarcer in evidence, and so far most efficient in a high frequency range of about $140 \mathrm{~Hz}$ (Moliadze, Antal, \& Paulus, 2010; Moliadze, Atalay, Antal, \& Paulus, 2012). Although offline effects in tACS may be a product of spike-time dependent plasticity resulting of the tuning of neural rhythms, evidence that these effects are dependent on the online action of the technique are not conclusive (Y.-Z. Huang et al., 2017; Veniero et al., 2015). In this thesis, tACS is used to target physiologically occurring theta-gamma phaseamplitude coupling at the left temporal cortex during the encoding phase of a verbal memory task.

\subsection{The modulation of episodic long-term memory by tES}

A growing set of experimental work supports tES-related modulatory effects on memory formation or retrieval, ranging from improvements to impairments, although frequently smallsized (K. Kim, Ekstrom, \& Tandon, 2016), with some reports of negative results (Braun, Sokoliuk, \& Hanslmayr, 2017). So far, the majority of the studies with tES and episodic long-term memory were conducted with tDCS. A review in 2012 (Manenti et al., 2012) showed the that most tDCS studies focused the stimulation on the DLPFC with relative success, generally improving verbal memory with anodal or impairing with cathodal tDCS, applied to the encoding or retrieval phase of a task. Another study with verbal material showed an improvement in memory performance 
when tDCS was applied to frontal and parietal regions of young participants during the retrieval phase of a task, but not during encoding (Manenti, Brambilla, Petesi, Ferrari, \& Cotelli, 2013). In another case, tDCS improved memory retrieval of non-verbal material and impaired verbal and non-verbal when applied to the parietal and pre-frontal cortices, respectively (Manuel \& Schnider, 2016). Nevertheless, these two studies suffered from clear pitfalls, as not comparing real against sham conditions or being single-blind, which can raise doubts about the plausibility of the effects. Two further studies showed that anodal and cathodal stimulation applied to the DLPFC during encoding respectively improved and impaired both verbal (A. H. Javadi \& Walsh, 2012) and pictorial (Zwissler et al., 2014) memory in recognition tasks. More recently, only anodal tDCS to the ventrolateral pre-frontal cortex was able to augment memory performance when applied to encoding, but no to retrieval, of verbal material (Medvedeva et al., 2018). Again, at the parietal cortex, anodal tDCS applied during retrieval in a verbal task improved performance (Pisoni et al., 2015).

Early studies applying oscillatory tES to modulate episodic memory used alternating currents with a DC-offset during sleep, and were able to influence verbal memory encoding (Marshall, Helgadóttir, Mölle, \& Born, 2006; Marshall, Kirov, Brade, Mölle, \& Born, 2011). However, these results could not be replicated during sleep (Eggert et al., 2013; Paßmann et al., 2016; Sahlem et al., 2015) and during wakefulness when applied after memory encoding, yet during learning the free recall of associated-words showed improvement (Kirov, Weiss, Siebner, Born, \& Marshall, 2009). More recent approaches with older humans and Alzheimer patients revealed a positive effect on memory of oscillatory tES applied during day-naps (J. Ladenbauer et al., 2016; X. J. Ladenbauer et al., 2017). With tACS, $140 \mathrm{~Hz}$ bilaterally applied at the dorsolateral pre-frontal cortex during the encoding of word pairs before sleep reduced overnight forgetting of 
learned material (Ambrus et al., 2015). In a different approach, Javadi et al. (2017a) used gamma tACS to the DLPFC during both encoding and retrieval to modulate memory reinstatement and could enhance verbal episodic memory. On the other hand, Braun and colleagues (2017) attempted to modulate verbal and non-verbal episodic memory formation in the alpha, beta and low gamma range, using a short-burst event-related approach. Results showed null-effects on memory performance for words and faces in a recognition paradigm. Taken together, the modulation of episodic memory by tES is so far bringing mixed results, with several research groups tracking for the optimal stimulation parameters that can bring reliable and replicable outcomes.

\subsection{Limitations of tES}

Although being generally well-received in the scientific community and experiencing a strong increase in its use on the last years (Polanía et al., 2018), transcranial electrical stimulation does not come without limitations and critics. TDCS and tACS studies have been showing replication issues and small effect sizes across motor, perception and cognitive fields (Chew, Ho, \& Loo, 2015; Emmerling et al., 2017; Horvath, Forte, \& Carter, 2014, 2015; Parkin, Bhandari, Glen, \& Walsh, 2018; Raffin \& Siebner, 2014), with notable inter- and intra-individual variability in the obtained results (Y.-Z. Huang et al., 2017). The major sources of variability to NIBS protocols have been pointed as intrinsic/biological factors and extrinsic/protocol factors. In the former, aspects such as age, gender, genetics, lifestyle, individual neuroanatomy and synaptic history, time of the day, arousal, substance use and additional unknown that may affect plasticity. In the latter, technical factors as tES parameters (polarity, intensity, duration, montage), study design, sample 
size, statistics, neuroimaging recordings and behavioral tasks directly impact the experimental outcomes(Guerra, López-Alonso, Cheeran, \& Suppa, 2017; Y.-Z. Huang et al., 2017).

In the cognitive domain, the widespread and simplistic use of tES to enhance cognitive functions as diverse as reading, decision making, speech, working memory and mathematical skills comes with at the expense of raising concerns in the quality and reliability of these studies (Parkin et al., 2015). Recently, Parkin et al. (2018) investigated the assumptions of classical tDCS parameters applied to cognitive processes. There, they tested the assumptions that a bilateral tDCS montage and a $2 \mathrm{~mA}$ would have the enhancing properties on cognition as showed by several studies. The results using TMS-generated MEPs to evaluate cortico-spinal excitability revealed null results for bilateral montages, adding up evidence to these concerns on the literature using such stimulation parameters for the purposes of cognitive enhancement.

In general, the field of tES research brings several promises of cognitive enhancement, although clear-cut designs and methodological shortcomings are a topic under current intensive discussion. Some suggestions were already drawn to make the most conclusive and less variable studies, when technically and financially possible: the use of neuronavigation, the experimental control of behavioral tasks, sites and frequencies, the use of neuroimaging, and the elaboration of clearer mechanistical models to the NIBS-induced changes. This so-called multi-method studies would take careful consideration about planning the stimulation targeting, adequate control conditions and reasonable sample sizes (Polanía et al., 2018). A recent panel of specialists (Bikson et al., 2018) brought on a list of recommendations to improve quality and reproducibility in tES research. They included electrode preparation and placement, operator training, protocol blinding and report, the use of computational models for spatial targeting and dose individualization, and the use of multi-modal approaches that include neuroimaging. 


\section{Targeting the left pre-frontal cortex during verbal episodic memory encoding with transcranial direct current stimulation}

The participation of the left pre-frontal cortex in episodic long-term memory encoding is well-stablished in the literature (Dickerson \& Eichenbaum, 2010). It is assumed that the region acts as a hub for semantic processing, and primarily actively executing organizational functions of monitoring and managing episodic memories (Eichenbaum, 2017). Accordingly, attempts to modulate this region's activity with transcranial direct current stimulation have been conducted in the last years, with moderate success (Dedoncker, Brunoni, Baeken, \& Vanderhasselt, 2016b). Nevertheless, the majority of the studies employ a typical bipolar (i.e., two electrodes) montage bilaterally placed at the left and right pre-frontal cortex (Dedoncker, Brunoni, Baeken, \& Vanderhasselt, 2016a). This sort of tES setting usually leads to less focal distribution of the generated electric-field, a potential confounding factor for the effects of absence of it (Hill, Rogasch, Fitzgerald, \& Hoy, 2017). In the first study (Lara, Knechtges, Paulus, \& Antal, 2017), we aimed to modulate memory performance by applying a focal multi-electrode tDCS based on a computational model to stablish our stimulation parameters, targeting primarily the dorso-lateral pre-frontal cortex (DLPFC). We expected to augment cortical excitability with anodal tDCS, and consequently, ignite verbal memory performance. Two groups of fifteen young healthy participants were stimulated continuously for 20 minutes, each group in a different timing step (first during encoding and second during retrieval). The behavioral assessment was conducted through a classical paired-associative verbal learning task in a cued-recall fashion. We found no significant effects of tDCS on memory performance on both of the measured groups. The possible factors for the obtained results are discussed on the published paper that follows. 
OPEN ACCESS

Edited by:

Gregor Thut,

University of Glasgow,

United Kingdom

Reviewed by:

Paulo Sérgio Boggio,

Mackenzie Presbyterian University,

Brazil

Michael Banissy,

Goldsmiths, University of London,

United Kingdom

*Correspondence:

Gabriel A. de Lara

gabriel.delara@med.uni-goettingen.de

Specialty section:

This article was submitted to

Perception Science,

a section of the journal

Frontiers in Neuroscience

Received: 09 May 2017

Accepted: 25 July 2017

Published: 08 August 2017

Citation:

Lara GAd, Knechtges PN, Paulus W and Antal A (2017) Anodal tDCS Over the Left DLPFC Did Not Affect the Encoding and Retrieval of Verbal

Declarative Information.

Front. Neurosci. 11:452

doi: 10.3389/fnins.2017.00452

\section{Anodal tDCS Over the Left DLPFC Did Not Affect the Encoding and Retrieval of Verbal Declarative Information}

\author{
Gabriel A. de Lara *, Philipp N. Knechtges, Walter Paulus and Andrea Antal \\ Department of Clinical Neurophysiology, University Medical Center Goettingen, Georg-August University of Goettingen, \\ Göttingen, Germany
}

Several studies imply that anodal transcranial direct current stimulation (tDCS) over the left dorsolateral prefrontal cortex (DLPFC) can modulate the formation of verbal episodic memories. The aim of this study was to test if tDCS through a multi-electrode Laplacian montage over the left DLPFC could differentially modulate declarative memory performance depending on the application phase. Two groups of healthy participants $(n=2 \times 15)$ received $1 \mathrm{~mA}$ anodal or sham stimulation for 20 min during the encoding or during the recall phase on a delayed cued-recall, using a randomized, double-blinded, repeated-measures experimental design. Memory performance was assessed at two time points: $10 \mathrm{~min}$ and $24 \mathrm{~h}$ after learning. We found no significant difference between anodal and sham stimulation with regard to the memory scores between conditions (stimulation during encoding or recall) or between time points, suggesting that anodal tDCS over the left DLPFC with these stimulation parameters had no effect on the encoding and the consolidation of associative verbal content.

Keywords: tDCS, verbal associative learning, verbal long-term memory, DLPFC

\section{INTRODUCTION}

Low-intensity transcranial electrical brain stimulation (TES) has the potential to further improve our knowledge about the functional and neural correlates of declarative memory, by directly manipulating the neural activity of targeted brain areas before or during the performance of a given task. Previous studies in this research field have found promising improvements in subjects' recognition of encoded material when transcranial direct current (tDCS), alternating current (tACS), or oscillatory tDCS was applied in either the learning and/or in the recognition phase (Marshall et al., 2006; Jacobson et al., 2012; Javadi et al., 2012; Javadi and Walsh, 2012; Ambrus et al., 2015; Pisoni et al., 2015). Among the above-mentioned techniques, tDCS is one of the most extensively used TES methods. It is thought that tDCS is capable of inducing polarity-dependent, relatively long-lasting changes in the human brain, probably either by de- or hyperpolarising neurons' resting membrane potentials and causing a reversible change in the balance of excitatoryinhibitory cortical activity (for recent reviews see Hartwigsen et al., 2015; Woods et al., 2016; Fertonani and Miniussi, 2017).

A meta-analysis of fMRI studies on episodic memory showed left lateralized effects for the encoding of verbal material, arguing in favor of the involvement of the prefrontal cortex (Kim, 2011). Additionally, results from non-invasive brain stimulation studies suggest that the left 
dorsolateral prefrontal cortex (DLPFC) may be involved in both the encoding and retrieval of verbal content (Manenti et al., 2012). Furthermore, several sources of recent experimental data indicate that the application of anodal tDCS over the left DLPFC during learning results in improvements in different cognitive tasks, including the encoding of semantic material (e.g., Brunoni and Vanderhasselt, 2014; Dedoncker et al., 2016b; Kim et al., 2016; Hill et al., 2017), although conflicting results were also reported (e.g., Tremblay et al., 2014). Further research considered that the stimulation timing might be critical (Dedoncker et al., 2016a, b; e.g., before or during the performance of the task), with the results usually showing a small, but significant, effect on accuracy and reaction time in working memory, when tested after the application of anodal tDCS.

A recent study tested the hypothesis that long-term associative-memory engrams are stored in an excitatoryinhibitory balance in neuronal ensembles. Learning is assumed to change synaptic strength, which is disrupted during this process, with the new excitatory connections being rebalanced afterwards by inhibitory GABAergic mechanisms (Barron et al., 2016). They showed that by unmasking inhibitory connections using anodal tDCS to downregulate cortical GABA concentration after learning, significant improvement could be obtained in associative memory, which correlated with a decreased GABA level in the targeted area.

To clarify whether anodal tDCS directed to the left prefrontal cortex could indeed significantly modulate the encoding or retrieval of verbal associative learning, we chose to apply 20 min of tDCS, as it constitutes common standard in the field for cognitive paradigms (Hill et al., 2017). For this we designed two experiments with different stimulation time points: in the first group, anodal tDCS was applied before and during learning, in order to augment learning-induced neuronal plasticity. In the second group, stimulation was administered before and during the recall phase $24 \mathrm{~h}$ after learning with the aim to rebalance inhibitory plasticity after learning, as previous studies showed that anodal tDCS can effectively decrease the GABA level (Stagg, 2014; Stagg et al., 2014; Barron et al., 2016). While after learning (and during "forgetting") the new excitatory connections are frequently rebalanced by inhibition, we hypothesized that the stimulation during the recall phase might induce enhanced memory performance by downregulating the increased GABA level, compared to sham stimulation, and similarly, during the encoding phase.

\section{MATERIALS AND METHODS}

\section{Participants}

Thirty healthy, young adult, right-handed, native German speakers with normal or corrected-to-normal vision were recruited, after giving their informed consent. They were assigned to two groups of 15 participants each (group 1: eight females, mean age $24.8 \pm 3.5$, age range 18-30; group 2: seven females, mean age $24.6 \pm 3$, age range 18-31). They had no history of neuropsychiatric or brain disorders. The participants were naive to the applied task and were reimbursed for their participation. The project was approved by the ethics committee of the University Medical Center Göttingen and was conducted accordingly to the Declaration of Helsinki. No participant reported adverse effects.

\section{Experimental Procedure}

A randomized, double-blind, repeated-measures, placebocontrolled design was used, with each participant taking part only in one experimental group. Every participant underwent two stimulation conditions (anodal tDCS and sham) with blocks of two experiments related to one condition (stimulation during encoding-group 1 or recall—group 2) separated by $24 \mathrm{~h}$, generating a total of four sessions (Figure 1). The first and the third sessions consisted of a (1) learning phase, combined with the application of tDCS in the group 1, (2) a 10-min pause, and (3) a first cued-recall. The second and fourth sessions were composed of a second cued-recall to assess memory overnight consolidation, combined with tDCS only in group 2. Each stimulation session was separated by at least a 5-day interval to avoid carryover effects. To minimize the well-known learning effect in word-list memory tasks, the order of real and placebo conditions were counterbalanced across participants. At the beginning of the first session, the subjects received written instruction about the task and were informed about the experimental procedures. The participants also filled in an additional indicators questionnaire and were debriefed after the stimulation sessions.

\section{Stimulation Protocol}

tDCS was delivered by using a certified NeuroConn Multichannel stimulator (Ilmenau, Germany). We used a set of five $3 \mathrm{~cm}^{2}$ rubber-round electrodes with Ten 20 paste as conductivity mean. Both of the groups received $1.0 \mathrm{~mA}$ of tDCS applied for $20 \mathrm{~min}$ continuously during the learning phase of the task (group 1) or during the second day's cued-recall (group 2). For group 1 , the stimulation was started $12 \mathrm{~min}$ before presentation of the learning material, and then continued during it (learning duration was $8 \mathrm{~min}$ ); for group 2, the stimulation started $15 \mathrm{~min}$ before and then continued during the cued-recall (which lasted $5 \mathrm{~min}$ ). During the real (anodal tDCS) and sham stimulation the current was ramped up for $10 \mathrm{~s}$ in the beginning until reaching the programmed intensity, and then ramped down for $10 \mathrm{~s}$ at the end. In the sham condition, the current was additionally applied for $30 \mathrm{~s}$ and then discontinued. The impedances were kept below the limit of $5 \mathrm{k} \Omega$ as measured by the device.

\section{Montage}

The positioning of the electrodes was standardized and kept constant across the experiments as suggested by modeling studies (Saturnino et al., 2015), with the plugs and cables always turned in a medial-to-lateral direction. The Laplacian multielectrode montage, designed to answer our research hypothesis, was composed of a central anodal electrode over the AF3 position (according to the international 10-20 EEG system) and four surrounding return electrodes with $6 \mathrm{~cm}$ distance from the central one, and $10 \mathrm{~cm}$ distance between the medial and lateral electrodes (Figure 2A). A realistic finite-element model (Figure 2B) to evaluate the extension and precision of 


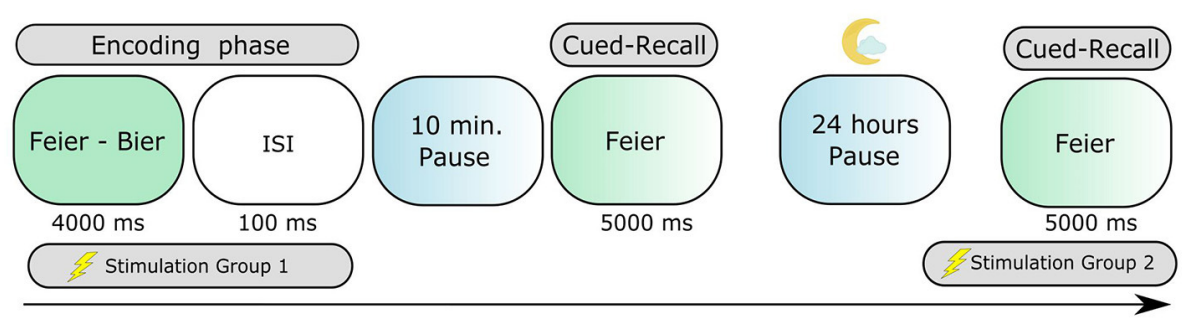

FIGURE 1 | The paired-associate learning task assessing episodic long-term memory. Participants learned 52 semantically-related German paired nouns. The cued-recall testing consisted in verbally expressing the second word of the pair, always $10 \mathrm{~min}$ and again $24 \mathrm{~h}$ after the encoding phase.
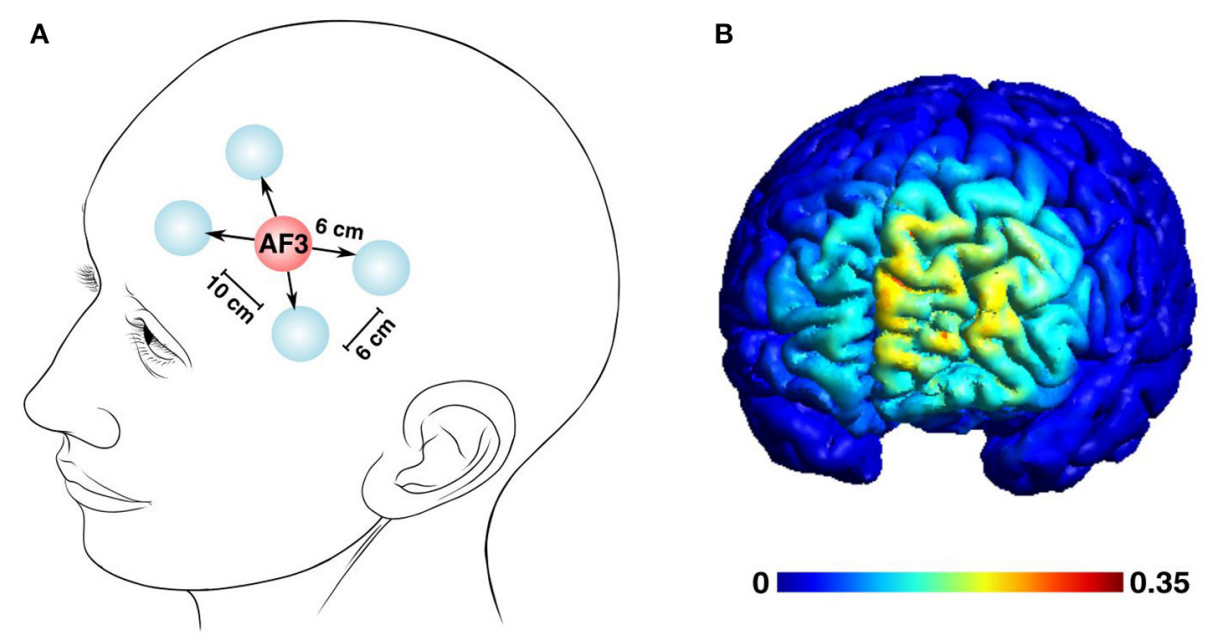

FIGURE 2 | The multi-electrode tDCS left pre-frontal montage and the estimated distribution of the tDCS-generated electric field. (A) A five-electrode Laplacian montage to deliver the current was centered over the AF3 position, surrounded by 4 return electrodes. The distances between the electrodes were set as follows: central and return electrodes, $6 \mathrm{~cm}$; adjacent return electrodes, $6 \mathrm{~cm}$; distance between the medial and lateral return electrodes, $10 \mathrm{~cm}$ (Human head modified from Patrick J. Lynch's illustration, distributed under a CC-BY 2.5 license.) (B) The estimated electric field distribution is color-coded to the intensity scale, with the maximum field strength reaching $0.35 \mathrm{mV} / \mathrm{mm}$.

our anatomical target and to estimate the distribution of the electric field was generated in SIMNIBS 2.0.1 (Thielscher et al., 2015). The model accounts for white matter anisotropy and the following conductivity for these anatomical components: scalp $(\sigma=0.465 \mathrm{~S} / \mathrm{m})$, bone $(\sigma=0.010 \mathrm{~S} / \mathrm{m})$, cerebrospinal fluid $(\sigma$ $=1.654 \mathrm{~S} / \mathrm{m})$, gray matter $(\sigma=0.275 \mathrm{~S} / \mathrm{m})$, and white matter $(\sigma=0.126 \mathrm{~S} / \mathrm{m})$. The tetrahedral volume mesh post-processing and visualization was generated through Gmsh (Geuzaine and Remacle, 2009).

\section{Task}

A verbal-associative learning task (Figure 1), shown in previous studies to be sensitive in the capture of effects of non-invasive brain stimulation in declarative memory (Marshall et al., 2006; Garside et al., 2015), was utilized in order to assess verbal episodic memory. In this paradigm, the participants were asked to memorize semantically related word-pairs presented one at a time. For each experimental condition, a different list with a total of 54 word-pairs composed of associated German nouns was presented on a monitor, where 8 (4 in the beginning and
4 in the end) of them were dummy pairs to buffer recency and primacy effects. The order of the lists was randomized across subjects and conditions. Each correct answer was granted two points, with one point given to late or partially correct (morphologically incorrect) answers, totaling 92 possible points (100\% performance) to score. The dummy pairs were excluded from the data analysis. The subjects were exposed to each word-pair for $4 \mathrm{~s}$ with an inter-stimulus interval of $100 \mathrm{~ms}$, thereby learning the list two times in a different, randomized order. With two different time delays (10 min and $24 \mathrm{~h}$ ), the participants' memory performance was subsequently tested with a cued-recall in a forward-recall manner, where each stimuli was presented for $5 \mathrm{~s}$. The stimuli in the two cuedrecalls were presented in two different, randomized orders. During the 10-min pause following the learning phase, the participants stayed seated and had no other activity or verbal interaction with the researcher. No feedback was given about the correctness of the answers. The task was conducted using Presentation software (Neurobehavioral Systems Inc., Albany, CA, USA). 


\section{Statistical Analysis}

In a first step, all the groups underwent null hypothesis significance testing to compare their behavioral performance in the task. As in the first experimental group two variables were non-normally distributed, a related-samples Wilcoxon signed rank test was employed in order to compare memory performance between sham and real tDCS, both for the first and second testing days. To rule out baseline differences that could impact the outcomes in our parallel-group design, a nonparametric independent-samples Mann-Whitney $U$-test was used to compare the sham condition performances on both days. In addition to the null hypothesis significance testing, we ran Bayesian analyses to verify the amount of evidence for the null or the alternative hypothesis given in our dataset (Rouder et al., 2009). One-sided JSZ Bayes Factors $\left(\mathrm{BF}_{01}\right)$ were computed in JASP (version 0.8.1.2) to estimate how likely the null hypothesis (there are no differences between the conditions) could be observed under the alternative hypothesis (there are differences), with a Cauchy prior width of 0.707 . We also calculated the effect size for all the real conditions compared with sham for the respective groups (Figure 3C). The calculations were performed with the Measures of Effect Size toolbox for MATLAB, which provides a corrected and unbiased Hedges'g estimation for small paired samples (Hentschke and Stuttgen, 2011).

\section{Arousal Levels and Sleep Quality Indicators}

To control for two variables that directly influence memory encoding and retrieval (Diekelmann and Born, 2010; Rutishauser et al., 2010), we asked the participants to report their arousal levels and sleep time and quality in the previous night (Table 1). The arousal was assessed on a self-report scale from 1 to 10 (1 $=$ very tired, $10=$ totally awake). Sleep quality was measured through self-report, including the number of hours subjects slept during the previous night (Likert scale, 1-5 points continuum; $1=$ very bad, $5=$ very good). All the indicators were analyzed using the non-parametric paired samples Wilcoxon signed rank test.

\section{RESULTS}

\section{Memory Accuracy}

The results with regard to memory performance in the pairedassociative learning task are summarized in Figure 3. The task permitted an absolute maximum numerical score of 92 points, and the results are plotted in original values for all days when memory accuracy was measured in a cued-recall fashion. In the first group $(n=15)$, where the participants received the stimulation during encoding, a small numerical difference in memory performance was observed between the real $(67.2 \pm$ $17.3)$ condition compared to sham $(67.8 \pm 16.8)$ stimulation in the first recall test, and also between sham $(66.5 \pm 17.1)$ and real tDCS $(64.7 \pm 16.4)$ on the second day of recall. The related-samples Wilcoxon signed rank test revealed no statistically significant effect of the stimulation condition, for either the first day $(Z=-0.057, p=0.955$, Hedges'g $=0.03$ ) or the second day $(Z=0.664, p=0.506$, Hedges'g $=0.10)$ of testing. The computed Bayes Factor showed moderate evidence in favor of the null hypothesis on the first cued-recall $\left(\mathrm{BF}_{01}=\right.$ 3.719), where the null hypothesis is 3.719 times more likely to be observed that the alternative hypothesis given this dataset. For the second cued-recall, Bayes Factor also showed moderate evidence for the null hypothesis $\left(\mathrm{BF}_{01}=3.237\right)$, i.e., it is 3.237 times more likely to be observed than the alternative given the present data.

For the second group $(n=15)$, where the participants received the tDCS during retrieval, the first cued-recall showed a slight numerical difference in memory performance between the anodal tDCS group (70.3 \pm 10.6$)$ compared to sham (71.4 \pm 8.3 ). On the delayed cued-recall, memory performance was also slightly different between the real stimulation $(70.0 \pm 11.6)$ compared to sham $(69.4 \pm 10.6)$. The related samples Wilcoxon signed rank test revealed no statistically significant differences in memory performance between sham and real tDCS for either the first $(Z=-0.711, p=0.477$, Hedges' $=0.11)$ or the second cued-recall $(Z=-0.566, p=0.572$, Hedges'g $=-0.04)$. Here, Bayes Factor showed moderate evidence in favor of the null hypothesis $\left(\mathrm{BF}_{01}=3.326\right)$ for the first cued-recall, where the null is 3.326 times more likely to be observed than the alternative. For the second cued-recall, Bayes Factor also showed moderate evidence for the null hypothesis $\left(\mathrm{BF}_{01}=3.737\right)$, being 3.737 times more likely to be observed given the actual data.

Moreover, the independent samples Mann-Whitney $U$-test revealed no significant difference in sham performance between the groups, for neither the first cued-recall $(U=112.500, p=$ $1.000)$, or for the second cued-recall $(U=119.500, p=0.771)$.

\section{Sleep Quality and Arousal Indicators}

A summary of the sleep quality and arousal data collected on the day of the experiments is reported in Table 1. In the first group (stimulation during encoding), the number of hours slept the night before the experimental session showed no significant difference between real and sham condition for session one or session two. There was also no significant difference in sleep quality report on the night before the experiments between real and sham for either of the sessions. Similarly, Wilcoxon signed rank test showed no significant difference in the arousal levels between sham and real stimulation for the first and second day of memory cued-recall testing.

For the second group (stimulation during retrieval), the amount of reported hours slept on the night before the experiments showed no significant impact on the results for either the real or sham conditions for any of the cuedrecall sessions. The sleep quality was not significantly different between real and sham stimulation before the first session, but significant difference was observed before the second session, where participants judged that they slept better before receiving sham stimulation $(p=0.008)$. A significant difference was detected in the arousal levels before the session between sham and real stimulation for the first day of memory cued-recall testing, where participants reported higher arousal levels in the real tDCS condition $(p=0.006)$, but not for the second cuedrecall. 


\section{A Group one: tDCS during encoding}

\section{Day One}

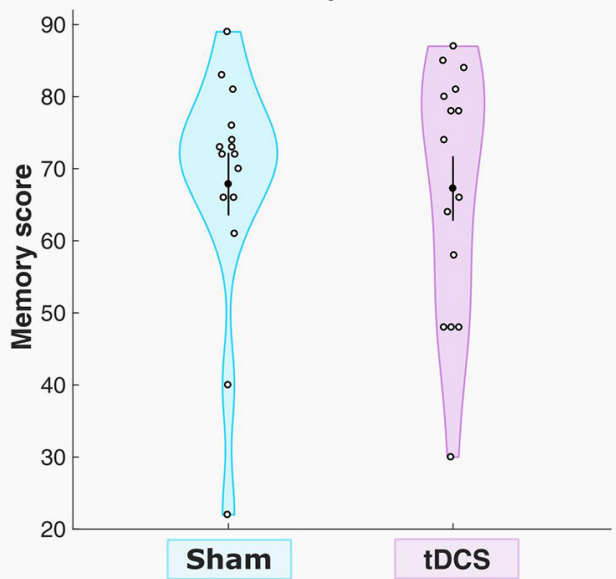

B Group two: tDCS during recall

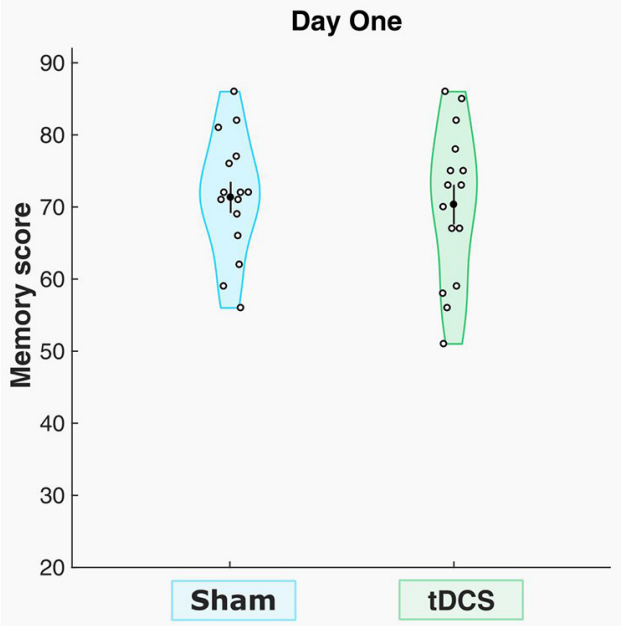

Day Two

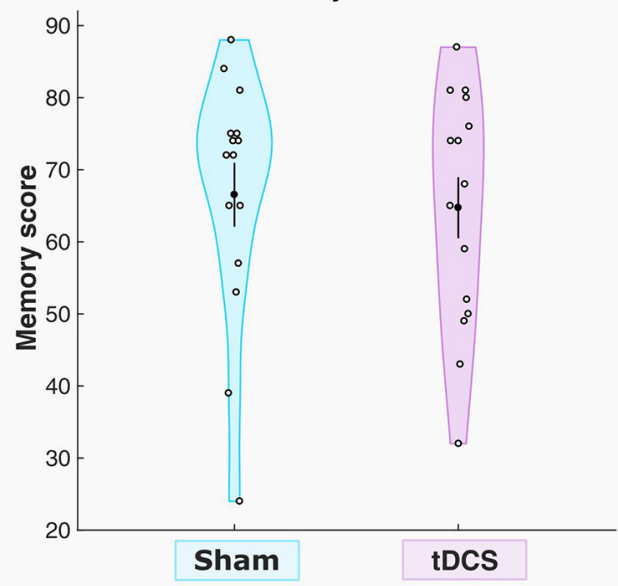

Day Two

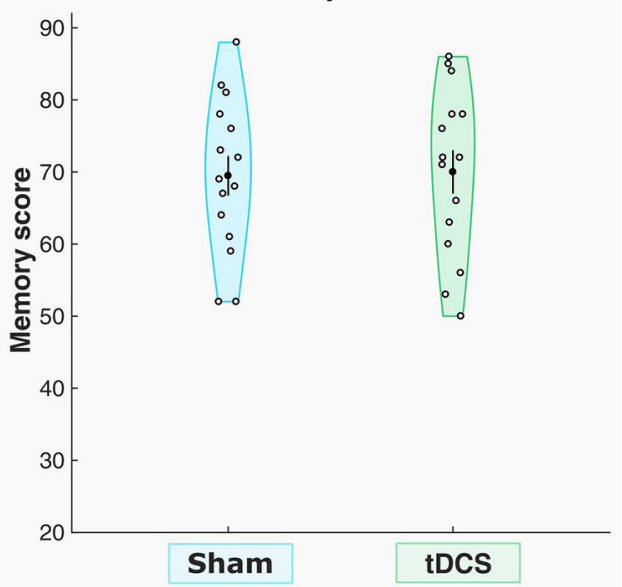

C

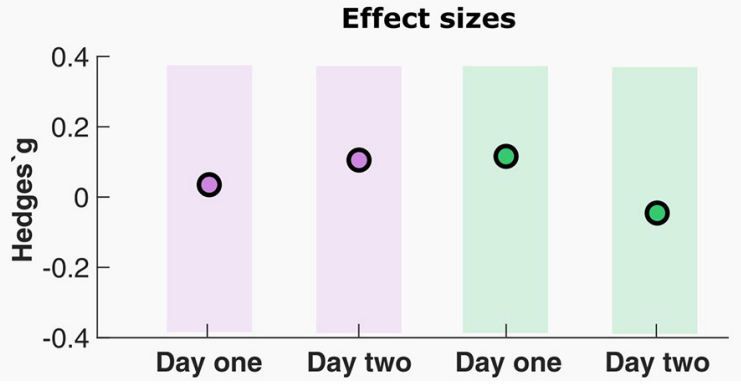

FIGURE 3 | tDCS had no significant effects on memory performance. The violin plots indicate the density of the sample distribution across the $y$-values. Mean and standard error of the mean (SEM) are shown in each plot as the black dot and the black line (A) Memory score for sham and real stimulation conditions for each participant in group one, day 1 and 2, respectively. (B) Memory score for sham and real stimulation conditions for each participant in group two, day 1 and 2 , respectively. (C) Effect sizes for the real tACS conditions across the two groups. 
TABLE 1 | Results for the sleep and arousal indicators.

\begin{tabular}{|c|c|c|c|}
\hline & Condition & Value & Test \\
\hline \multicolumn{4}{|l|}{ SLEEP } \\
\hline \multicolumn{4}{|l|}{ Group 1} \\
\hline \multirow[t]{6}{*}{ Amount of hours } & Session 1 & & $p=0.092$ \\
\hline & Real & $7.5 \pm 0.81$ & \\
\hline & Sham & $7.1 \pm 1.1$ & \\
\hline & Session 2 & & $p=0.223$ \\
\hline & Real & $7.2 \pm 0.9$ & \\
\hline & Sham & $6.7 \pm 1.5$ & \\
\hline \multirow[t]{6}{*}{ Sleep quality } & Session 1 & & $p=0.683$ \\
\hline & Real & $3.4 \pm 1.0$ & \\
\hline & Sham & $3.6 \pm 1.0$ & \\
\hline & Session 2 & & $p=0.666$ \\
\hline & Real & $3.9 \pm 0.8$ & \\
\hline & Sham & $3.9 \pm 1.0$ & \\
\hline
\end{tabular}

\section{Group 2}

Amount of hours

\section{Session 1 \\ Real \\ Sham \\ Session 2 \\ Real \\ Sham}

\begin{tabular}{llll}
\hline Sleep quality & Session 1 & & $p=0.490$ \\
& Real & $3.6 \pm 0.7$ & \\
& Sham & $3.4 \pm 0.9$ & $p=0.008$ \\
& Session 2 & & \\
& Real & $3.4 \pm 0.9$ & \\
& Sham & $4.3 \pm 0.6$ & \\
AROuSAL & & & \\
Group 1 & & & \\
First cued recall & Real & $7.1 \pm 1.2$ & \\
& Sham & $7.2 \pm 1.4$ & \\
\hline Second cued recall & Real & $7.4 \pm 0.9$ & \\
& Sham & $7.6 \pm 1.3$ & \\
\hline Group 2 & & & \\
First cued recall & Real & $7.9 \pm 1.1$ & \\
& Sham & $6.3 \pm 1.8$ & \\
\hline Second cued recall & Real & $7.0 \pm 1.4$ & $p=0.848$ \\
& Sham & $7.0 \pm 1.9$ & \\
\hline
\end{tabular}

\section{DISCUSSION}

In the present study we investigate the effect of anodal tDCS over the left DLPFC on verbal-associative long-term memory performance. Interestingly, none of the stimulation conditions (before-during learning or before-during the recall phase) resulted in a modification of performance compared to the subjects during sham $\mathrm{tDCS}$. Significant differences were observed between anodal and sham condition only in the sleep quality and arousal level in Group 2, however, without behavioral consequences.

Previous work has suggested that the excitatory-inhibitory balance in neuronal networks is disturbed during the learning of a new material (Song et al., 2000; Nabavi et al., 2014). In this period, when novel information is stored during the modification of excitatory synaptic strengths, anodal tDCS can have a beneficial effect and, consequently, augment the learning process. Indeed, data from numerous past experiments has implied that anodal tDCS can modify reaction time (for a review see Dedoncker et al., 2016b) or memory performance (for a recent review see: Hill et al., 2016) when administered in this critical period. However, the small effect sizes of previous studies, coupled with non-significant effects on several analyses, require cautious interpretation of these data. Moreover, since in the first experiment we fit the learning inside the last minutes of the stimulation protocol, homeostatic metaplastic effects could have driven the results toward a cancelation, as shown already when long-term potentiation-like brain stimulation protocols were applied prior to motor learning in humans (Jung and Ziemann, 2009).

With regard to the administration of anodal tDCS on the day after the verbal information encoding (experiment 2), we hypothesized that by decreasing the inhibitory rebalancing that is thought to take place after learning-induced increase in neuronal excitation (Froemke et al., 2007), a larger amount of semantic information would be recalled and memory performance would increase compared to sham stimulation. However, this was not the case and we were not able to replicate previous findings (Barron et al., 2016). Nevertheless, we can speculate that the timing of the stimulation in Group 2 might not have been ideal (e.g., the memory test should have occurred after the $20 \mathrm{~min}$ stimulation), as decreases in GABA levels were significantly lower after anodal tDCS applied at the primary motor cortex, but not during it, when compared to baseline measurements and to sham stimulation (Bachtiar et al., 2015). Another possible scenario is that the applied intensity might have been too low to generate an electric field strong enough to overcome the inhibitory effect. Nevertheless, the same intensity was used in previous research to successfully modulate GABA levels with anodal tDCS (Bachtiar et al., 2015; Barron et al., 2016).

Joyal and Fecteau (2016) reviewed studies that used tDCS in an attempt to modulate semantic processing. The data revealed a structured network correlated with this function, which included the inferior frontal gyrus, a region adjacent to the stimulated area but not directly targeted by it, a fact that can be related to the absence of significant behavioral outcomes. Moreover, we could speculate that the cathodal electrodes close to the lateral part of the frontal cortex may have driven this site to temporal inhibition. Additionally, findings on the cellular and network plasticity mechanisms that govern human learning and memory point to the fact that the search for a specific locus for the memory engram storage can be misleading, due to its possible widespread nature, i.e., the memory trace may be stored in connectivity patterns in different brain sites defined during encoding (Tonegawa et al., 2015). Therefore, targeting only one structure may be a limitation of each study with a design similar to the present one.

Furthermore, our target area might also not have been ideal. Indeed, multiple brain regions and not only the DLPFC are assumed to interact in order to coordinate verbal information 
processing, both for encoding and retrieval of declarative memories (Cabeza and Nyberg, 2000; Borst and Anderson, 2013; Pisoni et al., 2015). Besides, a fixed montage as chosen here can impact the study outcome. An individualized anatomical approach governed by neuronavigation to localize the DLPFC could optimize the results, in keeping with the known variability of brain anatomy as related to gender, aging, lateralization and pathological processes (Mylius et al., 2013). In addition, we employed electrode types that differed from the ones used in previous studies, that is, five smaller electrodes arranged in a Laplacian montage. Although, it was suggested by computational models and experimental studies that this sort of montage could effectively change cortical activity (Datta et al., 2009, 2012; Gbadeyan et al., 2016), the electric field induced by this montage is more focal and less deep compared to the one evoked by conventional pad electrodes.

Since we did not observe significant behavioral effects in the responses to tDCS, it would have been interesting to identify external and internal factors that might account for the negative results. Certainly, the variability in the cortical changes after tDCS that might result in the absence of group effects is a frequently discussed issue, particularly at the case of motor-evoked potentials responses after tDCS (e.g., Wiethoff et al., 2014). Many factors that could modulate responsiveness before, during or after tDCS have been identified (these being methodological-, investigator- and subject-related), however, until now, no consensus has been reached about the reasons underlying the between- and within-subject variability of tDCS effects. Although, it is difficult to compare directly, we believe that with regard to age, gender, educational level or sample size, this work did not differ from those published by previous research in tDCS and episodic memory.

So far, these studies in healthy participants have presented small effect sizes (Cohen's d of 0.04), with samples varying from 12 to 20 participants, with females outperforming male participants (Dedoncker et al., 2016a). Moreover, a metaanalysis that included only single-session protocols showed no significant effects of anodal tDCS on episodic memory (Dedoncker et al., 2016b). In another meta-analysis that included research with similar designs to the present work, only one study presented significant results, with a sample size of $n=16$ (Horvath et al., 2015). Furthermore, in a recently published work (Emmerling et al., 2017), anodal tDCS was applied to the right DLPFC in order to manipulate cognitive control. The authors used the same experimental conditions in two

\section{REFERENCES}

Ambrus, G. G., Pisoni, A., Primassin, A., Turi, Z., Paulus, W., and Antal, A. (2015). Bi-frontal transcranial alternating current stimulation in the ripple range reduced overnight forgetting. Front. Cell. Neurosci. 9:374. doi: 10.3389/fncel.2015.00374

Bachtiar, V., Near, J., Johansen-Berg, H., and Stagg, C. J. (2015). Modulation of GABA and resting state functional connectivity by transcranial direct current stimulation. Elife 4, 1-9. doi: 10.7554/eLife.08789

Barron, H. C., Vogels, T. P., Emir, U. E., Makin, T. R., O'shea, J., Clare, S., et al. (2016). Unmasking latent inhibitory connections in independent experimental groups (with 18 and 16 participants receiving anodal stimulation), and surprisingly, after positive results in the first experiment, they failed to replicate their own previous findings in the second. They admitted that although this could have been related to insufficient power, the mechanisms underlying tDCS at the neuronal level are far from being understood.

Taken together, contrary to previously published results, we have found no evidence that single-session anodal tDCS over the left DLPFC with the parameters used in this work has a reliable effect on encoding and retrieval of verbal information in healthy adult subjects. These findings further highlight the importance of uncovering the methodological factors that might underlie interindividual variability in response to tDCS, such as anatomical differences and electrode placement. Studies that combine behavioral outcomes with neurophysiological measures should systematically evaluate stimulation parameters and correlate them to effects in order to identify predictive factors. We also suggest that future studies should report not only the mean group data but also the individual performance data points. Moreover, to determine how far the negative results translate to a larger population, a higher number of participants is required.

\section{ETHICS STATEMENT}

This study was approved by the University Medical Center Göttingen Ethics Committee, under the title: Einfluss transkranieller Stromstimulation auf die kognitiven Funktionen. The project number is $12 / 04 / 2012$, and it is registered under the IFS 1716.

\section{AUTHOR CONTRIBUTIONS}

GL and AA designed the study. GL analyzed the data. PK and GL collected the data. GL, PK, WP, and AA wrote the paper.

\section{ACKNOWLEDGMENTS}

We thank Zsolt Turi and Ivan Alekseichuk for the discussions regarding the study design. We also thank Ivan Alekseichuk for providing the electric field distribution model. This work was supported by the Deutsche Forschungsgemeinschaft (PA 419/15-1) awarded to WP. We thank Christine Crozier for language editing of the manuscript. We acknowledge support by the Open Access Publication Funds of the Göttingen University. human cortex to reveal dormant cortical memories. Neuron 90, 191-203. doi: 10.1016/j.neuron.2016.02.031

Borst, J. P., and Anderson, J. R. (2013). Using model-based functional MRI to locate working memory updates and declarative memory retrievals in the fronto-parietal network. Proc. Natl. Acad. Sci. U.S.A. 110, 1628-1633. doi: 10.1073/pnas.1221572110

Brunoni, A. R., and Vanderhasselt, M. A. (2014). Working memory improvement with non-invasive brain stimulation of the dorsolateral prefrontal cortex: a systematic review and meta-analysis. Brain Cogn. 86, 1-9. doi: 10.1016/j.bandc.2014. 01.008 
Cabeza, R., and Nyberg, L. (2000). Neural bases of learning and memory: functional neuroimaging evidence. Curr. Opin. Neurol. 13, 415-421. doi: 10.1097/00019052-200008000-00008

Datta, A., Bansal, V., Diaz, J., Patel, J., Reato, D., and Bikson, M. (2009). Gyri-precise head model of transcranial direct current stimulation: Improved spatial focality using a ring electrode versus conventional rectangular pad. Brain Stimul. 2, 201-207, 207.e1. doi: 10.1016/j.brs.2009. 03.005

Datta, A., Truong, D., Minhas, P., Parra, L. C., and Bikson, M. (2012). Inter-individual variation during transcranial direct current stimulation and normalization of dose using MRI-derived computational models. Front. Psychiatry 3:91. doi: 10.3389/fpsyt.2012.00091

Dedoncker, J., Brunoni, A. R., Baeken, C., and Vanderhasselt, M. A. (2016a). The effect of the interval-between-sessions on prefrontal transcranial direct current stimulation (tDCS) on cognitive outcomes: a systematic review and meta-analysis. J. Neural Transm. 123, 1159-1172. doi: 10.1007/s00702-0161558-x

Dedoncker, J., Brunoni, A. R., Baeken, C., and Vanderhasselt, M. A. (2016b). A systematic review and meta-analysis of the effects of transcranial direct current stimulation (tDCS) over the dorsolateral prefrontal cortex in healthy and neuropsychiatric samples: influence of stimulation parameters. Brain Stimul. 9, 501-517. doi: 10.1016/j.brs.2016.04.006

Diekelmann, S., and Born, J. (2010). The memory function of sleep. Nat. Rev. Neurosci. 11, 114-126. doi: 10.1038/nrn2762

Emmerling, F., Martijn, C., Alberts, H. J. E. M., Thomson, A. C., David, B., Kessler, D., et al. (2017). The (non-) replicability of regulatory resource depletion: A field report employing non-invasive brain stimulation. PLOS ONE 12:e0174331. doi: 10.1371/journal.pone. 0174331

Fertonani, A., and Miniussi, C. (2017). Transcranial electrical stimulation. Neuroscientist 23, 109-123. doi: 10.1177/10738584166 31966

Froemke, R. C., Merzenich, M. M., and Schreiner, C. E. (2007). A synaptic memory trace for cortical receptive field plasticity. Nature 450, 425-429. doi: $10.1038 /$ nature 06289

Garside, P., Arizpe, J., Lau, C. I., Goh, C., and Walsh, V. (2015). Crosshemispheric alternating current stimulation during a nap disrupts slow wave activity and associated memory consolidation. Brain Stimul. 8, 520-527. doi: 10.1016/j.brs.2014.12.010

Gbadeyan, O., McMahon, K., Steinhauser, M., and Meinzer, M. (2016). Stimulation of dorsolateral prefrontal cortex enhances adaptive cognitive control: a high-definition transcranial direct current stimulation study. J. Neurosci. 36, 12530-12536. doi: 10.1523/JNEUROSCI.2450-1 6.2016

Geuzaine, C., and Remacle, J. F. (2009). Gmsh: a 3-D finite element mesh generator with built-in pre- and post-processing facilities. Int. J. Numer. Methods Eng. 79, 1309-1331. doi: 10.1002/nme.2579

Hartwigsen, G., Bergmann, T. O., Herz, D. M., Angstmann, S., Karabanov, A., Raffin, E., et al. (2015). Modeling the effects of noninvasive transcranial brain stimulation at the biophysical, network, and cognitive level. Prog. Brain Res. 222, 261-287. doi: 10.1016/bs.pbr.2015. 06.014

Hentschke, H., and Stuttgen, M. C. (2011). Computation of measures of effect size for neuroscience data sets. Eur. J. Neurosci. 34, 1887-1894. doi: 10.1111/j.1460-9568.2011.07902.x

Hill, A. T., Rogasch, N. C., Fitzgerald, P. B., and Hoy, K. E. (2017). Effects of prefrontal bipolar and high-definition transcranial direct current stimulation on cortical reactivity and working memory in healthy adults. Neuroimage 152, 142-157. doi: 10.1016/j.neuroimage.2017.03.001

Hill, A. T., Fitzgerald, P. B., and Hoy, K. E. (2016). Effects of anodal transcranial direct current stimulation on working memory: a systematic review and meta-analysis of findings from healthy and neuropsychiatric populations. Brain Stimul. 9, 197-208. doi: 10.1016/j.brs.2015. 10.006

Horvath, J. C., Forte, J. D., and Carter, O. (2015). Quantitative review finds no evidence of cognitive effects in healthy populations from single-session transcranial direct current stimulation (tDCS). Brain Stimul. 8, 535-550. doi: 10.1016/j.brs.2015.01.400
Jacobson, L., Goren, N., Lavidor, M., and Levy, D. A. (2012). Oppositional transcranial direct current stimulation (tDCS) of parietal substrates of attention during encoding modulates episodic memory. Brain Res. 1439, 66-72. doi: 10.1016/j.brainres.2011.12.036

Javadi, A. H., and Walsh, V. (2012). Transcranial direct current stimulation (tDCS) of the left dorsolateral prefrontal cortex modulates declarative memory. Brain Stimul. 5, 231-241. doi: 10.1016/j.brs.2011. 06.007

Javadi, A. H., Cheng, P., and Walsh, V. (2012). Short duration transcranial direct current stimulation (tDCS) modulates verbal memory. Brain Stimul. 5, 468-474. doi: 10.1016/j.brs.2011.08.003

Joyal, M., and Fecteau, S. (2016). Transcranial direct current stimulation effects on semantic processing in healthy individuals. Brain Stimul. 9, 682-691. doi: 10.1016/j.brs.2016.05.003

Jung, P., and Ziemann, U. (2009). Homeostatic and nonhomeostatic modulation of learning in human motor cortex. J. Neurosci. 29, 5597-5604. doi: 10.1523/JNEUROSCI.0222-09.2009

Kim, H. (2011). Neural activity that predicts subsequent memory and forgetting: a meta-analysis of 74 fMRI studies. Neuroimage 54, 2446-2461. doi: 10.1016/j.neuroimage.2010.09.045

Kim, K., Ekstrom, A. D., and Tandon, N. (2016). A network approach for modulating memory processes via direct and indirect brain stimulation: toward a causal approach for the neural basis of memory. Neurobiol. Learn. Mem. 134, 162-177. doi: 10.1016/j.nlm.2016. 04.001

Manenti, R., Cotelli, M., Robertson, I. H., and Miniussi, C. (2012). Transcranial brain stimulation studies of episodic memory in young adults, elderly adults and individuals with memory dysfunction: a review. Brain Stimul. 5, 103-109. doi: 10.1016/j.brs.2012.03.004

Marshall, L., Helgadóttir, H., Mölle, M., and Born, J. (2006). Boosting slow oscillations during sleep potentiates memory. Nature 444, 610-613. doi: $10.1038 /$ nature 05278

Mylius, V., Ayache, S. S., Ahdab, R., Farhat, W. H., Zouari, H. G., Belke, M., et al. (2013). Definition of DLPFC and M1 according to anatomical landmarks for navigated brain stimulation: Inter-rater reliability, accuracy, and influence of gender and age. NeuroImage 78, 224-232. doi: 10.1016/j.neuroimage.2013.03.061

Nabavi, S., Fox, R., Proulx, C. D., Lin, J. Y., Tsien, R. Y., and Malinow, R. (2014). Engineering a memory with LTD and LTP. Nature 511, 348-352. doi: $10.1038 /$ nature 13294

Pisoni, A., Turi, Z., Raithel, A., Ambrus, G. G., Alekseichuk, I., Schacht, A., et al. (2015). Separating recognition processes of declarative memory via anodal tDCS: boosting old item recognition by temporal and new item detection by parietal stimulation. PLOS ONE 10:e0123085. doi: 10.1371/journal.pone.01 23085

Rouder, J. N., Speckman, P. L., Sun, D., Morey, R. D., and Iverson, G. (2009). Bayesian $\mathrm{t}$ tests for accepting and rejecting the null hypothesis. Psychon. Bull. Rev. 16, 225-237. doi: 10.3758/PBR.16.2.225

Rutishauser, U., Ross, I. B., Mamelak, A. N., and Schuman, E. M. (2010). Human memory strength is predicted by theta-frequency phase-locking of single neurons. Nature 464, 903-907. doi: 10.1038/nature08860

Saturnino, G. B., Antunes, A., and Thielscher, A. (2015). On the importance of electrode parameters for shaping electric field patterns generated by tDCS. Neuroimage 120, 25-35. doi: 10.1016/j.neuroimage.2015.06.067

Song, S., Miller, K. D., and Abbott, L. F. (2000). Competitive Hebbian learning through spike-timing-dependent synaptic plasticity. Nat. Neurosci. 3, 919-926. doi: $10.1038 / 78829$

Stagg, C. J. (2014). Magnetic Resonance Spectroscopy as a tool to study the role of GABA in motor-cortical plasticity. Neuroimage 86, 19-27. doi: 10.1016/j.neuroimage.2013.01.009

Stagg, C. J., Bachtiar, V., Amadi, U., Gudberg, C. A., Ilie, A. S., Sampaio-Baptista, C., et al. (2014). Local GABA concentration is related to network-level resting functional connectivity. Elife 3:e01465. doi: 10.7554/eLife.01465

Thielscher, A., Antunes, A., and Saturnino, G. B. (2015). Field modeling for transcranial magnetic stimulation: a useful tool to understand the physiological effects of TMS? Conf. Proc. IEEE Eng. Med. Biol. Soc. 2015, 222-225. doi: 10.1109/EMBC.2015.7318340 
Tonegawa, S., Pignatelli, M., Roy, D. S., and Ryan, T. J. (2015). Memory engram storage and retrieval. Curr. Opin. Neurobiol. 35, 101-109. doi: 10.1016/j.conb.2015.07.009

Tremblay, S., Lepage, J.-F., Latulipe-Loiselle, A., Fregni, F., Pascual-Leone, A., and Théoret, H (2014). The Uncertain Outcome of Prefrontal tDCS. Brain Stimul. 7, 773-783. doi: 10.1016/j.brs.2014.10.003

Wiethoff, S., Hamada, M., and Rothwell, J. C. (2014). Variability in response to transcranial direct current stimulation of the motor cortex. Brain Stimul. 7, 468-475. doi: 10.1016/j.brs.2014.02.003

Woods, A. J., Antal, A., Bikson, M., Boggio, P. S., Brunoni, A. R., Celnik, P., et al. (2016). A technical guide to tDCS, and related non-invasive brain stimulation tools. Clin. Neurophysiol. 127, 1031-1048. doi: 10.1016/j.clinph.2015. 11.012
Conflict of Interest Statement: WP holds a patent on transcranial deep brain stimulation. He is on the scientific advisory board of Precisis AG.

The other authors declare that the research was conducted in the absence of any commercial or financial relationships that could be construed as a potential conflict of interest.

Copyright (c) 2017 Lara, Knechtges, Paulus and Antal. This is an open-access article distributed under the terms of the Creative Commons Attribution License (CC BY). The use, distribution or reproduction in other forums is permitted, provided the original author(s) or licensor are credited and that the original publication in this journal is cited, in accordance with accepted academic practice. No use, distribution or reproduction is permitted which does not comply with these terms. 


\section{Targeting the left temporal cortex during verbal episodic memory encoding with transcranial alternating current stimulation}

In the second study (Lara et al., 2018), we moved our target to the left temporal cortex. This time we aimed to interfere and manipulate with the temporal characteristics of the encoding process, and for that we chose tACS. Here, we attempted to profit on the naturally occurring thetagamma phase-amplitude coupling observations of previous neuroimaging studies (Hyafil et al., 2015) to build or scientific framework and hypotheses. It is still debated to which extend crossfrequency phase-amplitude coupling in different cortical sites is fundamental to successful episodic memory encoding, including at the lateral temporal cortex (Lega et al., 2016). Recently, a first study employed a theta-gamma cross-frequency tES waveform to the left pre-frontal cortex and was able to modulate working memory performance and cortical connectivity (Alekseichuk, Turi, Amador de Lara, Antal, \& Paulus, 2016). We further extend this idea to another memory modality and cortical area, and, attempting to modulate theta-gamma cross-frequency coupling during episodic memory encoding, we stimulated 72 healthy young participants divided into 3 groups with cross-frequency theta-gamma tACS. In one group, gamma bursts were rhythmically coupled to the peak of the theta cycle, whereas in a second group they were coupled to the trough of theta. In a third condition, as a control, the two frequencies were continuously intertwined for the entire theta cycle. Here, the same verbal associative learning task as in the previous chapter was utilized. When high gamma bursts were couplet to the trough of the theta cycle, memory was significantly impaired. This work adds new evidence to the participation of theta-gamma coupling to the physiology of verbal episodic memory encoding. 


\section{Accepted Manuscript}

Perturbation of theta-gamma coupling at the temporal lobe hinders verbal declarative memory

Gabriel Amador de Lara, Ivan Alekseichuk, Zsolt Turi, Albert Lehr, Andrea Antal, Walter Paulus

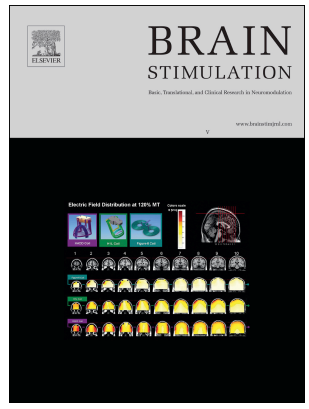

PII:

S1935-861X(17)31023-9

DOI:

10.1016/j.brs.2017.12.007

Reference: BRS 1165

To appear in: Brain Stimulation

Received Date: 8 September 2017

Revised Date: 21 November 2017

Accepted Date: 19 December 2017

Please cite this article as: Amador de Lara G, Alekseichuk I, Turi Z, Lehr A, Antal A, Paulus W, Perturbation of theta-gamma coupling at the temporal lobe hinders verbal declarative memory, Brain Stimulation (2018), doi: 10.1016/j.brs.2017.12.007.

This is a PDF file of an unedited manuscript that has been accepted for publication. As a service to our customers we are providing this early version of the manuscript. The manuscript will undergo copyediting, typesetting, and review of the resulting proof before it is published in its final form. Please note that during the production process errors may be discovered which could affect the content, and all legal disclaimers that apply to the journal pertain. 


\section{Perturbation of theta-gamma coupling at the}

2

3

4 Gabriel Amador de Lara ${ }^{1,}{ }^{*}$, Ivan Alekseichuk ${ }^{1}$, Zsolt Turi ${ }^{1}$, Albert Lehr ${ }^{1}$, Andrea Antal

$5 \quad$, Walter Paulus ${ }^{1}$

6 1. Department of Clinical Neurophysiology, University Medical Center Goettingen, 7 8

9

\author{
University of Goettingen, Robert-Koch-Straße 40, 37075 Göttingen, Germany.
}

*Correspondence: Gabriel Amador de Lara. Dept. of Clinical Neurophysiology, University Medical Center Goettingen, 37075 Göttingen, Germany. Phone: +49 05513919265. gabriel.delara@med.uni-goettingen.de

\section{Abbreviations}

PAC: Phase-amplitude cross-frequency coupling

tACS: transcranial alternating current stimulation

TGp-tACS: short bursts of high gamma tACS cycles that are coupled to the peak of the theta tACS cycle

TGt-tACS: short bursts of high gamma tACS that are coupled to the trough of the theta tACSwaveform

TGc-tACS: continuous high gamma and theta tACS

LTD: long-term potentiation

LTD: long-term depression

\section{temporal lobe hinders verbal declarative memory}




\section{Abstract}

Background: Phase-amplitude cross-frequency coupling (PAC) is characterized by the modulation of the power of a fast brain oscillation (e.g., gamma) by the phase of a slow rhythm (e.g., theta). PAC in different sub- and neocortical regions is known to underlie effective neural communications and correlates with successful long-term memory formation.

Objective/Hypothesis: The present work aims to extend earlier observational data, by probing the functional role of theta-gamma PAC in the left temporal cortex in humans during verbal longterm memory encoding.

Methods: In three double-blinded, placebo-controlled experiments $(n=72)$, we employed crossfrequency transcranial alternating current stimulation (tACS) to externally modulate ongoing PAC during a verbal-associative learning task. Three types of cross-frequency tACS protocols were used: bursts of high gamma tACS were coupled to the peak or trough of the theta tACS cycle, and a control condition where gamma tACS was continuously superimposed at theta tACS cycles.

Results: Gamma bursts coupled to the trough of theta tACS induced robust behavioral impairment in memory performance $(\mathrm{p}<0.01)$, whereas gamma burst coupled to the peak or continuously superimposed with theta tACS had no significant behavioral effects.

Conclusions: Our results demonstrate direct evidence regarding the importance of theta-gamma coupling in verbal long-term memory formation.

Keywords: tACS, verbal episodic memory, phase-amplitude cross-frequency coupling, longterm memory, temporal lobe 


\section{Introduction}

Complex cognitive functions, like declarative long-term memory, rely on large-scale distributed networks operating in precise spatiotemporal coordination. Neural oscillations dynamically coordinate the information flow between the medial temporal lobe and its projections to subcortical and neocortical sites, and have been shown to be crucial in associative memory formation [1-4]. Phase-amplitude cross-frequency coupling (PAC) is an electrophysiological phenomenon in which the phases of a slow oscillation selectively entrains the amplitude of a fast rhythm in a phase-locked manner [5,6].

Experiments in rodents' medial temporal lobe showed that, depending on the recording location (hippocampal fissure or CA1/CA3), the peak or trough in the theta cycle are thought to regulate the induction of long-term potentiation, and thus, memory encoding [7]. In essence, different phases of theta states would represent shifts in excitable periods that recruit functionally associated neuronal populations segregated into gamma cycles $[8,9]$. However, the relationship between theta-gamma PAC and memory is quite ambiguous: although evidence supports a positive relation in different cortical sites and its behavioral effect on long-term memory formation [10,11], a left-lateralized decrease in theta-gamma PAC was also found during the successful encoding of associative verbal and pictorial information $[12,13]$.

In order to mimic physiologically occurring PAC characteristics, we used cross-frequency transcranial alternating current stimulation (tACS), a non-invasive brain stimulation method that can causally probe the functional role of cross-frequency coupling by externally injecting oscillatory electrical current into the brain $[14,15]$. It is hypothesized that tACS can entrain the endogenous activity, i.e. induce changes in the amplitude and phase, and thus modulate neuronal firing synchronicity that rise as detectable brain rhythms [16,17]. In a recent work, theta-gamma 
cross-frequency tACS applied to the left pre-frontal cortex was able to increase visual spatial working memory performance on a two n-back match-to-sample task [18].

Here, we aimed to externally modulate PAC at the human temporal lobe during the memory formation stage of a verbal paired associated learning task. For this purpose, we utilized two cross-frequency theta-gamma tACS protocols: short bursts of high gamma tACS cycles were coupled to the peak (TGp-tACS; experiment one) or trough (TGt-tACS; experiment two) of the theta tACS cycle (Figure 1D). In addition, we introduced a control group in which the gamma frequency was continuously modulated along the theta cycle (TGc-tACS; experiment three). High gamma frequency was chosen due to its association with successful verbal memory encoding in the hippocampus and left temporal lobe in humans $[19,20]$. High gamma oscillation coupled with theta was also detected in rats and human's medial temporal lobe and neocortical structures during episodic memory encoding [21-23].

We hypothesized that TGp-tACS would magnify gamma oscillations and PAC at higher excitability phases, which would facilitate successful memory encoding by inducing LTP-like effects. On the other hand, an increase in PAC at the lower excitability theta phases (TGt-tACS) would impede memory encoding, reinforcing LTD-like plasticity mechanisms. LTD or LTP-like processes were already shown to be facilitated by different protocols with rhythmic stimulation, mainly when applied to different theta phases [5,24]. By coupling gamma bursts to different excitability phases in theta cycle, we attempted to alter normal PAC regimes when compared to sham stimulation. In the control group, gamma oscillations were modulated continuously along the theta wave (TGc-tACS), meaning no phase coupling specificity. From a dynamical systems theory perspective, this can be treated as a weak coupling, where the modulatory force of the slow rhythm is weak enough for the fast rhythm to stay present during the whole slow oscillation 
cycle, rendering the phase-amplitude relationship unsubstantial [25]. For that, we hypothesized that no significant differences in memory performance would be generated. We found that TGttACS induced a robust behavioral impairment in memory performance compared to the active sham condition, whereas TGp- and TGc-tACS produced no significant behavioral effects. Our findings provide direct evidence for the importance of theta-gamma coupling in memory formation and its external modulation by TG-tACS.

\section{Materials and Methods}

\section{Participants}

A total of 72 healthy, right-handed, young adult, native German speakers with normal or corrected-to-normal vision were recruited for the study, after giving their signed informed consent. They were randomly distributed into three experiments, each of which consisted of 24 participants, with 12 males and 12 females (experiment one: mean age $23.5 \pm 3.1$, age range 1832; experiment two: mean age $24.3 \pm 2.9$, age range 19-29; experiment three: mean age $23.2 \pm$ 2.2 , age range 19-27). They reported no history of neuropsychiatric disorders, drug-dependency, or neurological abnormalities. The participants were naïve to the memory test applied and were reimbursed for their participation. The experiments were approved by the Ethics Committee of the University Medical Center Goettingen, Germany, and conducted in agreement with the Declaration of Helsinki.

\section{Experimental procedures}

The study utilized a double-blind, randomized, placebo-controlled, parallel group design, where each participant took part in one experiment with two conditions (real stimulation and active sham). Every participant took part in four sessions. The first and the third sessions 
consisted of a (1) learning phase combined with the application of real or active sham tACS, (2) a ten minutes pause and (3) a cued-recall test. The second and fourth sessions occurred 24 hours after learning and consisted of a second cued-recall to assess overnight memory retention. Each experimental condition was separated by an at least a 5-day interval between stimulation sessions. To minimize the learning-to-learn effect on memorizing word lists that could interfere in the study outcome, the stimulation conditions were placed in a counterbalanced order across participants. The data collector was blinded with regard to the stimulation conditions by another researcher, who replaced the condition labels with dummy codes. In the beginning of the first experimental session, the subjects received a written instruction about the task and were informed about the stimulation procedures.

\section{Stimulation protocol}

The transcranial alternating current was delivered by means of a CE-certified Neuroconn multichannel stimulator (neuroConn $\mathrm{GmbH}$, Ilmenau, Germany). The stimulation protocol was in agreement with the present ethical guidelines [26]. We used a $3 \mathrm{~cm}^{2}$ rubber-round electrode with the Ten20 paste as the conductive mean. In all groups, for the real tACS protocol, a sinusoidal alternating current of $1 \mathrm{~mA}$ (peak-to-baseline) was applied for 10 minutes continuously during the learning phase of the task. As demonstrated by intracranial recordings during transcranial AC stimulation in humans, the stimulation intensity applied in this work can generate electric fields strong enough $[27,28]$ to modulate neuronal activity according to in vitro and in vivo studies $[29,30]$. During the real and active sham stimulation, the current was ramped up for 10 seconds in the beginning until the programmed intensity was reached, with a ramp down of 10 seconds in the end; but in the active sham condition, a current was delivered for 30 seconds and then discontinued by using a ramp-down of 10 seconds. The active sham stimulation consisted of a 
139 protocol already demonstrated to mimic the cutaneous sensations related to the real transcranial

140 electrical stimulation, although not leading to detectable behavioral after-effects [31]. The

141 impedances were kept below $10 \mathrm{k} \Omega$ as measured by the device. The positioning of the electrodes

142 was standardized and kept constant across the experiments as suggested by modelling studies

143 [32], with the plugs and cables always turned to an anterior-to-posterior direction.

In this work, the employed cross-frequency stimulation comprised two components

145 (figure 1D): in the first and second experiments, a slow, continuous $5 \mathrm{~Hz}$ theta wave $(0.75 \mathrm{~mA}$ 146 peak-to-baseline) modulated bursts of the $80 \mathrm{~Hz}$ gamma $(0.5 \mathrm{~mA}$ peak-to-baseline, $50 \mathrm{~ms}$

147 duration) fast component coupled with the peak and trough of each theta cycle, respectively; in

148 the third experiment, both components were merged into a continuous superimposed theta-

149 gamma wave, with the theta being the envelope of the gamma wave. There were no DC offsets in

150 the waveforms (which could lead to confounding effects). The temporal coordination of the

151 components was generated with a specific hardware and verified in the stimulator with an

152 oscilloscope.

153 Montage

The three-electrode montage was derived from a finite-element model to focus the electric

155 field distribution over the left temporal lobe. It was composed of a stimulation electrode over T7

156 and two return electrodes over $\mathrm{FPz}$ and $\mathrm{T} 8$, according to the international 10-20 EEG system

157 (Figure 1B). In order to estimate the distribution of the electric field, a realistic model (Figure 1C) was generated from the SIMNIBS 2.0.1 package [33,34]. This MRI-derived, finite element model took into account white matter anisotropy and conductivity [35] for the following anatomical compartments: scalp $(\sigma=0.465 \mathrm{~S} / \mathrm{m})$, bone $(\sigma=0.010 \mathrm{~S} / \mathrm{m})$, cerebrospinal fluid $(\sigma=$ 
161

162

163

164

165

166

167

168

169

170

171

172

173

174

175

176

177

178

179

180

181

182

$1.654 \mathrm{~S} / \mathrm{m})$, grey matter $(\sigma=0.275 \mathrm{~S} / \mathrm{m})$ and white matter $(\sigma=0.126 \mathrm{~S} / \mathrm{m})$. The tetrahedral volume mesh post-processing and visualization was done with Gmsh [36].

\section{Task}

Based on previous studies [37,38], a paired-associative learning task (Figure 1A) was employed to asses verbal episodic memory, using the study-test method, in which the participants studied a list of semantically related word-pairs composed of associated German nouns. For the two stimulation conditions (real or active sham), two independent word lists were used, each of which containing a total of 54 word-pairs. The order of the word lists was counterbalanced between stimulation conditions and participants. Within the word lists, the presentation order of the word-pairs was randomized for each word-list presentation, stimulation condition, participant and experiment. The stimuli were presented horizontally at the center of a 17" computer screen, written in white Arial with a font size 18 over a black background. The participants seated comfortably in chair placed at a $90 \mathrm{~cm}$ distance from the monitor. In order to facilitate the learning of the large amount of the to-be-encoded word-pairs, during the encoding phase (tACS + learning), the participants were presented one word list twice immediately after each other. From the 54 learned word-pairs, 8 ( 4 in the beginning and 4 in the end) of them were dummy items to buffer primacy and recency effects, which were excluded from the data analysis. Particularly, the recency (serial position) effect is accounted for the working memory component in the performance, which justifies the data exclusion. The subjects were exposed to each word pair for 5 seconds with an inter-stimulus interval of 100 milliseconds. The learning phase was followed by a 10-minute-long pause, during which the participants stayed quiet and performed no other activity. 
Each learned word list was tested in two different time points (10 minutes and 24 hours

184

185

186

187

188

189

190

191

192

193

194

195

196

197

198

199

200

201

202

203

204

205

206 after encoding) using a cued-recall in a forward-recall fashion, in which the order of the stimuli in the two test moments was randomized. In the testing phase, each recall trial lasted 5 seconds with the stimulus constantly being presented on the monitor. Participants responded verbally. Their responses were noted by the investigator in a standardized experimental sheet. The task correction criteria included granting one point to late answers (i.e., provided outside of the $5 \mathrm{~s}$ recall interval of a given trial) or partially correct answers (e.g., plural instead of singular), and two points for each correct answer, totaling a possible point score of 92. In addition, a different scoring criterion was used to test the validity of our procedure. In this second scoring approach, partially correct answers where considered as full hits, and late answers as misses. The results from the second scoring approach conceptually replicated the results from the first scoring approach (see supplementary material). No feedback was given during and after the recall tests regarding the correctness of their answers. The task was controlled using Presentation software (version 0.71, Neurobehavioral Systems Inc, Albany, CA., USA).

\section{Data analysis}

All the experiments were submitted to null hypothesis significance testing to compare the behavioral performance in the task. In experiment one, three variables (real stimulation day one and active sham stimulation days one and two) showed a non-normal distribution. For this, a nonparametric related-samples Wilcoxon signed-rank test was employed to compare sham and real tACS conditions in all the experimental groups. Bonferroni correction was used within experiments for correcting for multiple comparisons. A parametric independent-samples Kruskal-Wallis test was used to check if there were significant differences between the active sham conditions. To complement the applied null hypothesis significance testing, we estimated 
the effect size (Figure 3) for all the real conditions compared with active sham for the respective experiments. The calculations were performed with the Measures of Effect Size toolbox for MATLAB, that uses standardized mean difference and corrects for bias in cases of small sample sizes [39]. We additionally clustered our participants in three groups depending on whether their performance during active stimulation surpassed, decreased or remained the same when compared to the active sham condition.

\section{Arousal levels, cutaneous sensations and sleep quality indicators}

To control for the variables which can impact memory performance, we asked the participants to report their arousal levels, cutaneous sensations and the number of hours they had slept on the night before the experiment day (Table 1). The arousal degree was self-report on a scale going from 1-10 ( 1 = very tired, $10=$ totally awake $)$. Wilcoxon signed-ranks test was applied to assess differences on both of the indicators within groups, and the Independentsamples Kruskal-Wallis test when comparing the same indicators between groups.

We also accounted for a possible effect of cutaneous sensations/discomfort between the stimulation conditions and groups. The participants were asked whether they felt any skin sensation during the stimulation (by using an 11-point Likert-scale; 0 - not at all, to 10 - very strong). The related-samples Wilcoxon signed-rank test was applied to assess differences between real and active sham tACS within groups, whereas the independent-samples KruskalWallis test was used to compare cutaneous sensation between the groups.

\section{Results}

For the first experiment, we hypothesized that the modulation of the gamma bursts nested into the theta wave peaks (TGp-tACS) during the memory encoding would improve subsequent 
memory performance by enhancing theta-gamma coupling at theta high excitability phases [18].

The memory performance across the sample (Figure 2A) was numerically higher, although it was not statistically significant, in the real TGp-tACS condition $(70.1 \pm 14.3$ mean score; Hedges' $\mathrm{g}=$ .12) compared to the active sham $(68.2 \pm 15.8$ mean score $)$ for the first cued-recall $(\mathrm{Z}=.732, p=$ .928, Bonferroni corrected, Wilcoxon signed-rank test). Similar improvement also occurred with real TGp-tACS $(66.7 \pm 16.2$ mean score, Hedges' $\mathrm{g}=.15)$ compared to active sham stimulation (64 \pm 17.3 mean score) on the second cued-recall $(\mathrm{Z}=1.116, p=.528$, Bonferroni corrected, Wilcoxon signed-rank test). We further clustered the participants based on whether they had an increase or decrease in memory performance in response to real TGp-tACS (Figure 4A). No clear predominance of the effect was observed on the first cued-recall, while a slight numerically better performance was present on the second cued-recall. Additionally, we asked the participants before the experiments about their arousal level and the number of hour of sleep they got, factors that could impact memory encoding and retrieval performance [40,41]. No significant grouplevel differences were reported (Table 1).

In the second experiment, we hypothesized that the modulation of the gamma bursts nested into the theta wave troughs (TGt-tACS) would decrease memory performance. This decrease consistently occurred across the study volunteers (Figure 2B). On both cued-recalls, a consistent drop in memory performance was observed (79 \% and $75 \%$ of the subjects, respectively, Figure 4B). On the first cued-recall $(\mathrm{Z}=-2.844, p=.008$, Bonferroni corrected, Wilcoxon signed-rank test), real TGt-tACS $(62.3 \pm 15.4$ mean score, Hedges' $\mathrm{g}=-.57)$ impaired memory formation compared to the active sham stimulation $(71.3 \pm 15.2$ mean score). The same effect was observed on the second cued-recall $(\mathrm{Z}=-2.831, p=.010$, Bonferroni corrected, Wilcoxon signed-rank test), where real TGt-tACS (59.5 \pm 16.6 mean score, Hedges' $\mathrm{g}=-.49)$ led to a decrease in memory performance compared to active sham stimulation $(68.0 \pm 16.7$ mean 
score). The arousal and amount-of-sleep reports showed no significant differences between real and active sham stimulation on either of the cued-recalls (Table 1). The consistent memory impairment scores on two separate days indicate that the process of memory encoding was disrupted.

To additionally control for the phase relationships between theta and high gamma oscillations during declarative long-term memory formation, a third experiment was conducted, in which gamma oscillations were modulated continuously along the theta wave (TGc-tACS), meaning no phase coupling specificity (Figure 1D). We hypothesized that a weaker form of cross-frequency coupling would have no impact on memory formation and, hence, on memory performance. Confirming this hypothesis (Figure $2 \mathrm{C}$ ), we found no significant difference $(\mathrm{Z}=$ $.098, p=1.0$, Bonferroni corrected, Wilcoxon signed-rank test) between real TGc-tACS (65.4 \pm 11.3 mean score, Hedges' $\mathrm{g}=.05)$ and the active sham stimulation $(64.6 \pm 13.5$ mean score $)$ on the first cued-recall, or between real TGc-tACS $(62.7 \pm 12.4$ mean score, Hedges' $g=.05)$ and the sham $(63.5 \pm 13.5$ mean score $)$ on the second cued-recall $(\mathrm{Z}=-.200, p=1.0$, Bonferroni corrected, Wilcoxon signed-rank test ). The performance clustering also did not show a clear predominance of any effect (Figure 4C). Again, there was no significant difference between the conditions in terms of self-reported arousal levels and hours slept on the night prior to the experiment (Table 1).

To further test whether fluctuations in individual performances could drive the difference in the outcomes between the stimulation conditions, we compared specifically the performance under the active sham tACS conditions between the three experimental groups. An independentsamples Kruskal-Wallis test revealed no significant difference between the active sham conditions on either the first $(\mathrm{H}(2)=3.545, p=.170)$ or the second cued-recall $(\mathrm{H}(2)=1.159, p=$ 
.560). The comparison of arousal and sleep reports for real and active sham tACS across the three experiments (Table 1) demonstrated no significant differences among them, excluding the possible influences of these variables fluctuation on memory performance.

The cutaneous sensations/discomfort comparison was conducted to identify differences between the stimulation conditions (active sham vs. real tACS) and groups. The skin sensation reported on the real tACS session was compared between the groups with the independentsamples Kruskall-Wallis test, which revealed no significant difference across the groups $(\mathrm{H}(2)=$ 1.612, $p=.447)$. We also tested if discomfort could account for differences between the conditions. We compared the skin sensation during the real and sham sessions within each group by using the related-samples Wilcoxon signed rank test. No significant difference was found for the TGp-tACS group $(\mathrm{Z}=-.451, p=.652)$, for the TGt-tACS $(\mathrm{Z}=.200, p=.841)$ and for the TGc-tACS $(\mathrm{Z}=.500, p=.617)$. These results suggest no detectable effect of the stimulationrelated skin discomfort on memory performance.

\section{Discussion}

The objective of this study was to investigate verbal episodic memory formation by targeting CFC between theta and high gamma oscillations at the temporal lobe. Cross-frequency tACS protocols, as used here, can further extend correlative evidence deriving from observation studies, towards a direct link between the theta-gamma cross-frequency hypothesis and cognition [18]. Due to its preponderance in processing and storing verbal content within the memory network [42], we targeted the left temporal lobe by three tACS CFC protocols. Our behavioral analysis revealed that short bursts of gamma tACS coupled with the trough of the theta tACS cycle significantly impaired verbal long-term memory performance. 
PAC between theta and gamma oscillations regulates inter- and intra-areal spike-time dependent plasticity that underlie learning mechanisms and the organizational pattern of encoding information [43-46]. In subdural recordings over left cortical areas in epilepsy patients, theta to high gamma coupling was present in $84.3 \%$ of the electrodes for multiple tasks, including semantic processing and episodic memory [23]. The theta-gamma PAC was also investigated in the context of sequential temporal encoding of single visual items, where magnetoencephalography revealed a robust nesting of gamma power to different theta phases associated with the serial position of the learned items in multiple structures of the MTL [13]. Additionally, direct recordings from the MTL in rodents and humans suggest that CFC between theta-low gamma might be involved in memory retrieval, whereas theta-high gamma coupling could be directly involved in encoding [21,22]. The CFC phenomenon, and a possible phase preference of high gamma amplitude in relation to the theta cycle during encoding, set the framework for our hypothesis on the role of theta-gamma coupling in the temporal lobe in declarative long-term memory.

The impairment of memory performance in our TGt-tACS experiment suggests the hindering of naturally occurring theta-gamma coupling characteristics, which is known to coordinate long-term memory encoding, demonstrating a clear sensitivity to this type of coupling. To understand the lack of memory improvement, we consider the following scenarios. In healthy young adults with intact cognitive functions, a ceiling effect in learning may occur more likely as compared to the likelihood of deterioration, regardless of whether electrical brain stimulation is applied or not; as well as optimized brain functions predominantly respond to external perturbations with impairment $[47,48]$. In line with this, a recent study, in which left or right medial temporal lobe was stimulated invasively using electrical pulses with a frequency of $50 \mathrm{~Hz}$, revealed only an impairment, but not an improvement, in verbal and spatial memory domains 
[49]. Also, a long and continuous stimulation as used here is more prone to induce a functional disruption, rather than enhancement [50], as patterned stimulation protocols could be more aligned with the physiological fluctuations of neuronal activity than the continuous ones, although showing variability issues in the outcomes [51,52]. This may be due to the fact that theta-gamma PAC typically fluctuates in a limited time window during memory encoding, as, in general, brain rhythms are transient in nature [53].

An alternative explanation may be related to the lack of agreement in the experimental data regarding the presence of CFC and its phase structure in successful memory encoding, a topic of constant debate on the field of neuroscience. In the verbal episodic memory domain, intracranial recordings revealed differences in PAC during episodic memory formation between hippocampal and neocortical recording sites [54]. While the hippocampus exhibited maximum PAC between both slow delta/theta $(2.5-5 \mathrm{~Hz})$ and low and high gamma rhythms, the lateral temporal lobe showed a different oscillatory pattern; that is, theta wave $(4-9 \mathrm{~Hz})$ modulated mainly low gamma activity. Future tACS research can explore the involvement of theta-low gamma PAC during verbal memory encoding. In addition, a recent study using a paired-verbal memory task has linked successful memory encoding to decreased rather than to increased PAC between theta and high gamma in the inferior frontal gyrus, and theta and low gamma in the left temporal lobe [12]. Nevertheless, the electrophysiological signal detected in the temporal site with intracranial recordings was classified as a sharp waveform deflection, not as a synchronized gamma oscillation nested to theta. Based on these last findings, we would expect functional impairment in all three TG-tACS experiments; however, our results clearly provided evidence for verbal memory impairment only in the TGt-tACS experiment.

The results from the realistic finite element model indicate the highest field strength allocated in the left temporal lobe (Figure 1C). As to the anatomical precision of our model- 
371 Conflict of interest: W.P. holds a patent on transcranial deep brain stimulation. He is on the

driven TG-tACS, the present results can be explained by at least two scenarios. First, it is possible that our findings are due to the direct effect of TG-tACS over the left lateral temporal lobe. This account is supported by experimental data, which correlated theta-gamma coupling in this region with verbal long-term memory encoding [54]. In addition, modulation of the left lateral temporal cortex could affect medial temporal lobe activity via the functional connections to it, also known as a "proxy" stimulation effect demonstrated by earlier studies [50,55]. Medial temporal lobe structures that connect to lateral temporal sub-regions, namely the anterior hippocampus and perirhinal/entorhinal cortices, were shown to encode verbal content as a part of the episodic memory network [56-61]. Second, we cannot rule out the intriguing possibility that TG-tACS, although to a lesser extent, reached deeper areas such as the medial temporal lobe structures, possibly via the cortico-spinal fluid [28]. Thus, it is possible that either direct or indirect stimulation of the medial temporal lobe with TG-tACS could be responsible for the present results.

Few limitations of the present study include the absence of electrophysiological recordings before and after TG-tACS, thus the resulting network dynamics remains to be characterized. Second, endogenous brain oscillation are transient in nature, however, the present paradigm utilized continuous stimulation for 10 minutes. This limitation requires the investigation of a temporally coordinated, event-related form of tACS.

Taken together, we demonstrated that the perturbation of theta-gamma coupling at the temporal lobe hinders declarative memory formation, by showing the feasibility of externally modulating verbal episodic memory encoding with theta-gamma cross-frequency stimulation.

372 scientific advisory board of EBS technologies and Precisis AG. 


\section{Acknowledgments}

We thank Christine Crozier for language editing of the manuscript. This work was supported by the Deutsche Forschungsgemeinschaft (PA 419/15-1) awarded to WP.

\section{References}

[1] Dickerson BC, Eichenbaum H. The episodic memory system: neurocircuitry and disorders. Neuropsychopharmacology 2010;35:86-104. doi:10.1038/npp.2009.126.

[2] Preston AR, Eichenbaum H. Interplay of Hippocampus and Prefrontal Cortex in Memory. Curr Biol 2013;23:R764-73. doi:10.1016/j.cub.2013.05.041.

[3] Thut G, Miniussi C, Gross J. The functional importance of rhythmic activity in the brain. Curr Biol 2012;22:R658-63. doi:10.1016/j.cub.2012.06.061.

[4] Siegel M, Donner TH, Engel AK. Spectral fingerprints of large-scale neuronal interactions. Nat Rev Neurosci 2012;13:20-5. doi:10.1038/nrn3137.

[5] Canolty RT, Knight RT. The functional role of cross-frequncy coupling. Trends Cogn Sci 2010;14:506-15. doi:10.1016/j.tics.2010.09.001.The.

[6] Fell J, Axmacher N. The role of phase synchronization in memory processes. Nat Rev Neurosci 2011;12:105-18. doi:10.1038/nrn2979.

[7] Hasselmo ME. What is the function of hippocampal theta rhythm? - Linking bahavioral data to phasic properties of field potential and unit recording data. Hippocampus 2005;15:936-49. doi:10.1002/hipo.20116.

[8] Buzsáki G, Anastassiou C a., Koch C. The origin of extracellular fields and currents EEG, ECoG, LFP and spikes. Nat Rev Neurosci 2012;13:407-20. doi:10.1038/nrn3241.

[9] Hanslmayr S, Staresina BP, Bowman H. Oscillations and Episodic Memory: Addressing the Synchronization/Desynchronization Conundrum. Trends Neurosci 2016;39:16-25. doi:10.1016/j.tins.2015.11.004.

[10] Lisman JE, Jensen O. The Theta-Gamma Neural Code. Neuron 2013;77:1002-16. doi:10.1016/j.neuron.2013.03.007.

[11] Friese U, Köster M, Hassler U, Martens U, Trujillo-Barreto N, Gruber T. Successful memory encoding is associated with increased cross-frequency coupling between frontal theta and posterior gamma oscillations in human scalp-recorded EEG. Neuroimage 2013;66:642-7. doi:10.1016/j.neuroimage.2012.11.002. 
[12] Vaz AP, Yaffe RB, Wittig JH, Inati SK, Zaghloul KA. Dual origins of measured phaseamplitude coupling reveal distinct neural mechanisms underlying episodic memory in the human cortex. Neuroimage 2017;148:148-59. doi:http://dx.doi.org/10.1101/084194.

[13] Heusser AC, Poeppel D, Ezzyat Y, Davachi L. Episodic sequence memory is supported by a theta-gamma phase code. Nat Neurosci 2016;19:1374-83. doi:10.1038/nn.4374.

[14] Helfrich RF, Herrmann CS, Engel AK, Schneider TR. Different coupling modes mediate cortical cross-frequency interactions. Neuroimage 2016;140:76-82. doi:10.1016/j.neuroimage.2015.11.035.

[15] Helfrich RF, Knight RT. Oscillatory Dynamics of Prefrontal Cognitive Control. Trends Cogn Sci 2016;20:916-30. doi:10.1016/j.tics.2016.09.007.

[16] Antal A, Herrmann CS. Transcranial Alternating Current and Random Noise Stimulation: Possible Mechanisms. Neural Plast 2016;2016:1-12. doi:10.1155/2016/3616807.

[17] Miniussi C, Harris JA, Ruzzoli M. Modelling non-invasive brain stimulation in cognitive neuroscience. Neurosci Biobehav Rev 2013;37:1702-12. doi:10.1016/j.neubiorev.2013.06.014.

[18] Alekseichuk I, Turi Z, Amador de Lara G, Antal A, Paulus W. Spatial Working Memory in Humans Depends on Theta and High Gamma Synchronization in the Prefrontal Cortex. Curr Biol 2016;26:1513-21. doi:10.1016/j.cub.2016.04.035.

[19] Sederberg PBP, Schulze-Bonhage A, Madsen JR, Bromfield EB, Litt B, Brandt A, et al. Gamma oscillations distinguish true from false memories. Psychol Sci 2007;18:927-32. doi:10.1111/j.1467-9280.2007.02003.x.Gamma.

[20] Sederberg PB, Schulze-Bonhage A, Madsen JR, Bromfield EB, McCarthy DC, Brandt A, et al. Hippocampal and neocortical gamma oscillations predict memory formation in humans. Cereb Cortex 2007;17:1190-6. doi:10.1093/cercor/bhl030.

[21] Colgin LL. Theta-gamma coupling in the entorhinal-hippocampal system. Curr Opin Neurobiol 2015;31:45-50. doi:10.1016/j.conb.2014.08.001.

[22] Colgin LL. Rhythms of the hippocampal network. Nat Rev Neurosci 2016;17:239-49. doi:10.1038/nrn.2016.21.

[23] Canolty RT, Edwards E, Dalal SS, Soltani M, Nagarajan SS, Berger MS, et al. High Gamma Power is Phase-Locked to Theta Oscillations in Human Neocortex. Science 2006;313:1626-8. doi:10.1126/science.1128115.High.

[24] Hyman JM, Wyble BP, Goyal V, Rossi CA, Hasselmo ME. Stimulation in hippocampal region CA1 in behaving rats yields long-term potentiation when delivered to the peak of theta and long-term depression when delivered to the trough. J Neurosci 2003;23:1172531. doi:23/37/11725 [pii].

[25] Hyafil A, Giraud A, Fontolan L, Gutkin B. Neural Cross-Frequency Coupling : Connecting Architectures, Mechanisms , and Functions. Trends Neurosci 2015;38:725-40. doi:10.1016/j.tins.2015.09.001. 
[26] Antal A, Alekseichuk I, Bikson M, Brockmöller J, Brunoni AR, Chen R, et al. Low intensity transcranial electric stimulation: Safety, ethical, legal regulatory and application guidelines. Clin Neurophysiol 2017;128:1774-809. doi:10.1016/j.clinph.2017.06.001.

[27] Opitz A, Falchier A, Yan C, Yeagle E, Linn G. Spatiotemporal structure of intracranial electric fields induced by transcranial electric stimulation in human and nonhuman primates. Sci Rep 2016;6:1-11. doi:10.1101/053892.

[28] Huang Y, Liu AA, Lafon B, Friedman D, Dayan M, Wang X, et al. Measurements and models of electric fields in the in vivo human brain during transcranial electric stimulation. Elife 2017;6:e18834. doi:10.7554/eLife.18834.

[29] Reato D, Rahman A, Bikson M, Parra LC. Effects of weak transcranial alternating current stimulation on brain activity-a review of known mechanisms from animal studies. Front Hum Neurosci 2013;7:687. doi:10.3389/fnhum.2013.00687.

[30] Ali MM, Sellers KK, Frohlich F. Transcranial Alternating Current Stimulation Modulates Large-Scale Cortical Network Activity by Network Resonance. J Neurosci 2013;33:11262-75. doi:10.1523/JNEUROSCI.5867-12.2013.

[31] Ambrus GG, Al-Moyed H, Chaieb L, Sarp L, Antal A, Paulus W. The fade-in - Short stimulation - Fade out approach to sham tDCS - Reliable at $1 \mathrm{~mA}$ for naïve and experienced subjects, but not investigators. Brain Stimul 2012;5:499-504. doi:10.1016/j.brs.2011.12.001.

[32] Saturnino GB, Antunes A, Thielscher A. On the importance of electrode parameters for shaping electric field patterns generated by tDCS. Neuroimage 2015;120:25-35. doi:10.1016/j.neuroimage.2015.06.067.

[33] Thielscher A, Antunes A, Saturnino GB. Field modeling for transcranial magnetic stimulation: A useful tool to understand the physiological effects of TMS? Proc Annu Int Conf IEEE Eng Med Biol Soc EMBS 2015;2015-Novem:222-5. doi:10.1109/EMBC.2015.7318340.

[34] Opitz A, Paulus W, Will A, Thielscher A. Anatomical determinants of the electric field during transcranial direct current stimulation. Neuroimage 2015;109:2. doi:10.1016/j.neuroimage.2015.01.033.

[35] Opitz A, Windhoff M, Heidemann RM, Turner R, Thielscher A. How the brain tissue shapes the electric field induced by transcranial magnetic stimulation. Neuroimage 2011;58:849-59. doi:10.1016/j.neuroimage.2011.06.069.

[36] Geuzaine C, Remacle JF. Gmsh: A 3-D finite element mesh generator with built-in preand post-processing facilities. Int J Numer Methods Eng 2009;79:1309-31. doi:10.1002/nme.2579.

[37] Rasch B, Pommer J, Diekelmann S, Born J. Pharmacological REM sleep suppression paradoxically improves rather than impairs skill memory. Nat Neurosci 2009;12:396-7. doi:10.1038/nn.2206. 
Slow Oscillation Enhances Memory. Neuron 2013;78:545-53. doi:10.1016/j.neuron.2013.03.006.

[39] Hentschke H, Stüttgen MC. Computation of measures of effect size for neuroscience data sets. Eur J Neurosci 2011;34:1887-94. doi:10.1111/j.1460-9568.2011.07902.x.

[40] Diekelmann S, Born J. The memory function of sleep. Nat Rev Neurosci 2010;11:114-26. doi:10.1038/nrn2762.

[41] Rutishauser U, Ross IB, Mamelak AN, Schuman EM. Human memory strength is predicted by theta-frequency phase-locking of single neurons. Nature 2010;464:903-7. doi:10.1038/nature08860.

[42] Kim H. Neural activity that predicts subsequent memory and forgetting: A meta-analysis of 74 fMRI studies. Neuroimage 2011;54:2446-61. doi:10.1016/j.neuroimage.2010.09.045.

[43] Düzel E, Penny WD, Burgess N. Brain oscillations and memory. Curr Opin Neurobiol 2010;20:245-57. doi:10.1016/j.conb.2010.01.004.

[44] Jutras MJ, Buffalo E a. Synchronous neural activity and memory formation. Curr Opin Neurobiol 2010;20:150-5. doi:10.1016/j.conb.2010.02.006.

[45] Buzsáki G, Wang X-J. Mechanisms of Gamma Oscillations. Annu Rev Neurosci 2012;35:203-25. doi:10.1146/annurev-neuro-062111-150444.

[46] Hanslmayr S, Staudigl T. How brain oscillations form memories - A processing based perspective on oscillatory subsequent memory effects. Neuroimage 2014;85:648-55. doi:10.1016/j.neuroimage.2013.05.121.

[47] Furuya S, Klaus M, Nitsche MA, Paulus W, Altenmu E. Ceiling Effects Prevent Further Improvement of Transcranial Stimulation in Skilled Musicians. J Neurosci 2014;34:13834-9. doi:10.1523/JNEUROSCI.1170-14.2014.

[48] Alekseichuk I, Pabel SC, Antal A, Paulus W. Intrahemispheric theta rhythm desynchronization impairs working memory. Restor Neurol Neurosci 2017;35:147-58. doi:10.3233/RNN-160714.

[49] Jacobs J, Miller J, Lee SA, Coffey T, Watrous AJ, Sperling MR, et al. Direct Electrical Stimulation of the Human Entorhinal Region and Hippocampus Impairs Memory. Neuron 2016;92:983-90. doi:10.1016/j.neuron.2016.10.062.

[50] Kim K, Ekstrom AD, Tandon N. A network approach for modulating memory processes via direct and indirect brain stimulation: Toward a causal approach for the neural basis of memory. Neurobiol Learn Mem 2016;134:162-77. doi:10.1016/j.nlm.2016.04.001.

[51] Hamada M, Hanajima R, Terao Y, Arai N, Furubayashi T, Inomata-Terada S, et al. Quadro-pulse stimulation is more effective than paired-pulse stimulation for plasticity induction of the human motor cortex. Clin Neurophysiol 2007;118:2672-82. doi:10.1016/j.clinph.2007.09.062. 
[52] Suppa A, Huang YZ, Funke K, Ridding MC, Cheeran B, Di Lazzaro V, et al. Ten Years of Theta Burst Stimulation in Humans: Established Knowledge, Unknowns and Prospects. Brain Stimul 2016;9:323-35. doi:10.1016/j.brs.2016.01.006.

[53] Jones SR. When brain rhythms aren't "rhythmic": implication for their mechanisms and meaning. Curr Opin Neurobiol 2016;40:72-80. doi:10.1016/j.conb.2016.06.010.

[54] Lega B, Burke J, Jacobs J, Kahana MJ. Slow-Theta-to-Gamma Phase-Amplitude Coupling in Human Hippocampus Supports the Formation of New Episodic Memories. Cereb Cortex 2016;26:268-78. doi:10.1093/cercor/bhu232.

[55] Wang JX, Rogers LM, Gross EZ, Ryals AJ, Dokucu ME, Brandstatt KL, et al. Targeted enhancement of cortical-hippocampal brain networks and associative memory. Science (80- ) 2014;345:1054-7. doi:10.1126/science.1252900.

[56] Ranganath C, Heller A, Cohen MX, Brozinsky CJ, Rissman J. Functional connectivity with the hippocampus during successful memory formation. Hippocampus 2005; 15:9971005. doi:10.1002/hipo.20141.

[57] Wagner K, Frings L, Halsband U, Everts R, Buller A, Spreer J, et al. Hippocampal functional connectivity reflects verbal episodic memory network integrity. Neuroreport 2007;18:1719-23. doi:10.1097/WNR.0b013e3282f0d3c5.

[58] McLaren DG, Sperling RA, Atri A. Flexible modulation of network connectivity related to cognition in alzheimer's disease. Neuroimage 2014;100:544-57. doi:10.1016/j.neuroimage.2014.05.032.

[59] Ranganath C, Ritchey M. Two cortical systems for memory-guided behaviour. Nat Rev Neurosci 2012;13:713-26. doi:10.1038/nrn3338.

[60] Blankenship SL, Redcay E, Dougherty LR, Riggins T. Development of hippocampal functional connectivity during childhood. Hum Brain Mapp 2017;38:182-201. doi:10.1002/hbm.23353.

[61] Kahn I, Andrews-Hanna JR, Vincent JL, Snyder AZ, Buckner RL. Distinct cortical anatomy linked to subregions of the medial temporal lobe revealed by intrinsic functional connectivity. J Neurophysiol 2008;100:129-39. doi:10.1152/jn.00077.2008.

\section{Figures and table legends}

Figure 1. Experimental parameters: (A) The verbal paired-associative learning task. Participants leaned 54 associated German nouns while receiving TG-tACS. The memory performance was tested twice in a cued-recall manner: the first test occurred 10 minutes after learning and the second test 24 hours after learning. (B) Stimulation montage: transcranial alternating current was delivered over the left temporal region (T7, international EEG 10-20 
system) with two return electrodes over the right temporal (T8) and frontal (Fpz) areas. Note that the amount of power provided by the summed energy of the components in each cycle corresponded to $2 \mathrm{~mA}$ peak-to-peak. (C) Realistic, anisotropic, finite-element computational head model of the electric field distribution in the brain, including the following compartments: scalp, bone, cerebrospinal fluid, grey and white matter. Colored circles in upper figures represent the tACS electrodes locations. Bottom right presents only field strength distribution in grey matter compartment. The field strength is color-coded from 0 to $0.6 \mathrm{mV} / \mathrm{mm}$. (D) Cross-frequency protocols for tACS where $80 \mathrm{~Hz}$ gamma bursts were (1) coupled with the peaks (TGp-tACS), (2) with the troughs (TGt-tACS), or (3) continuously presented during the $5 \mathrm{~Hz}$ theta wave cycles (TGc-tACS).

Figure 2: Episodic memory performance. Violin plots with individual data points indicate the scores in the verbal paired-associative learning task. (A) Active sham and real TGp-tACS for the first experiment $(80 \mathrm{~Hz}$ gamma bursts at the peak of the $5 \mathrm{~Hz}$ theta cycle). (B) Active sham and real TGt-tACS for the second experiment $(80 \mathrm{~Hz}$ gamma bursts at the trough of the $5 \mathrm{~Hz}$ theta cycle). (C) Active sham and real TGc-tACS for the third experiment (80 Hz gamma bursts continuously modulated by $5 \mathrm{~Hz}$ theta cycles). Mean and standard error of the mean are indicate by the filled black dot and the vertical black line, respectively. Horizontal brackets point statistically significant differences according to paired samples null significance hypothesis testing $(* \mathrm{p}<0.01$; Bonferroni corrected).

Figure 3. Effect sizes. Cross-frequency tACS protocols in three experimental groups. Values in the $\mathrm{Y}$ axis represent an unbiased Hedges' g estimator of the standardized mean performance 
compared to sham stimulation. The $\mathrm{X}$ axis presents the two cued-recall tests for each of the experimental groups.

Figure 4. Clustered memory performance. Proportion of volunteers that demonstrated increase or decrease in memory performance. Left column indicates the first cued-recall (10 min after learning and TG-tACS), and right the second cued-recall (24 hours after learning). Blue areas represent increase, orange areas represent decrease, and grey areas represent equal performance between real and active sham tACS.

Table 1. Sleep hours and arousal indicators. Comparisons between real and active sham tACS within experiments for the amount of hours slept and the self-reported arousal level before the memory test, both on the first and second experimental days. Wilcoxon signed ranks test revealed no significant differences for either indicator. Comparing the same indicators between experiments, again for the real and sham conditions and for both of the experimental days, the independent-samples Kruskal-Wallis test showed no significant differences. The value column indicates mean \pm standard deviation. 
Table 1.

\begin{tabular}{|c|c|c|c|}
\hline & Condition & Value & Test \\
\hline \multicolumn{4}{|l|}{ Sleep } \\
\hline \multicolumn{4}{|c|}{ Experiment 1 (peak) } \\
\hline \multirow[t]{4}{*}{ Number of hours } & $\begin{array}{l}\text { First day } \\
\text { real }\end{array}$ & $7.1 \pm 0.9$ & $p=.79$ \\
\hline & active sham & $7.2 \pm 1.0$ & \\
\hline & Second day & $74+11$ & \\
\hline & active sham & $7.0 \pm 1.0$ & \\
\hline \multicolumn{4}{|c|}{ Experiment 2 (trough) } \\
\hline \multirow[t]{5}{*}{ Number of hours } & First day & & \\
\hline & $\begin{array}{l}\text { real } \\
\text { active sham }\end{array}$ & $\begin{array}{l}7.2 \pm 0.9 \\
6.9 \pm 1.5\end{array}$ & \\
\hline & Second day & & \\
\hline & real & $7.2 \pm 1.1$ & $\mathrm{p}=.30$ \\
\hline & active sham & $7.4 \pm 1.3$ & \\
\hline \multicolumn{4}{|c|}{ Experiment 3 (continuous) } \\
\hline \multirow[t]{5}{*}{ Number of hours } & First day & & \\
\hline & real & $7.4 \pm 0.6$ & $\mathrm{p}=.92$ \\
\hline & & $7.4 \pm 0.7$ & \\
\hline & & $7.7 \pm 0.8$ & $\mathrm{p}=.95$ \\
\hline & active sham & $7.6 \pm 1.0$ & \\
\hline \multicolumn{4}{|l|}{ Arousal } \\
\hline \multicolumn{4}{|l|}{ Experiment 1} \\
\hline \multirow[t]{2}{*}{ First day } & & $6.8 \pm 1.8$ & $\mathrm{p}=.97$ \\
\hline & active sham & $6.8 \pm 1.3$ & \\
\hline \multirow[t]{2}{*}{ Second day } & real & $7.0 \pm 1.5$ & $\mathrm{p}=.42$ \\
\hline & active sham & $6.8 \pm 1.5$ & \\
\hline \multicolumn{4}{|l|}{ Experiment 2} \\
\hline \multirow[t]{2}{*}{ First day } & real & $6.6 \pm 1.1$ & $\mathrm{p}=.73$ \\
\hline & active sham & $6.5 \pm 1.5$ & \\
\hline \multirow[t]{2}{*}{ Second day } & real & $6.7 \pm 1.5$ & $\mathrm{p}=.14$ \\
\hline & active sham & $7.2 \pm 1.1$ & \\
\hline \multicolumn{4}{|l|}{ Experiment 3} \\
\hline \multirow[t]{2}{*}{ First day } & real & $7.4 \pm 1.1$ & $\mathrm{p}=.18$ \\
\hline & active sham & $6.8 \pm 1.5$ & \\
\hline \multirow[t]{2}{*}{ Second day } & real & $7.6 \pm 1.1$ & $\mathrm{p}=.96$ \\
\hline & active sham & $7.6 \pm 1.4$ & \\
\hline
\end{tabular}




\section{Between experiments}

\section{Arousal}

First day

real

active sham

Second day

real

active sham

$$
\begin{aligned}
& p=.06 \\
& p=.58 \\
& p=.14 \\
& p=.30
\end{aligned}
$$

Sleep hours

First day

Second day

real

active sham

real

active sham $\mathrm{p}=.61$

$\mathrm{p}=.37$

$\mathrm{p}=.62$

$\mathrm{p}=.09$ 
(A) 2 Study cycles

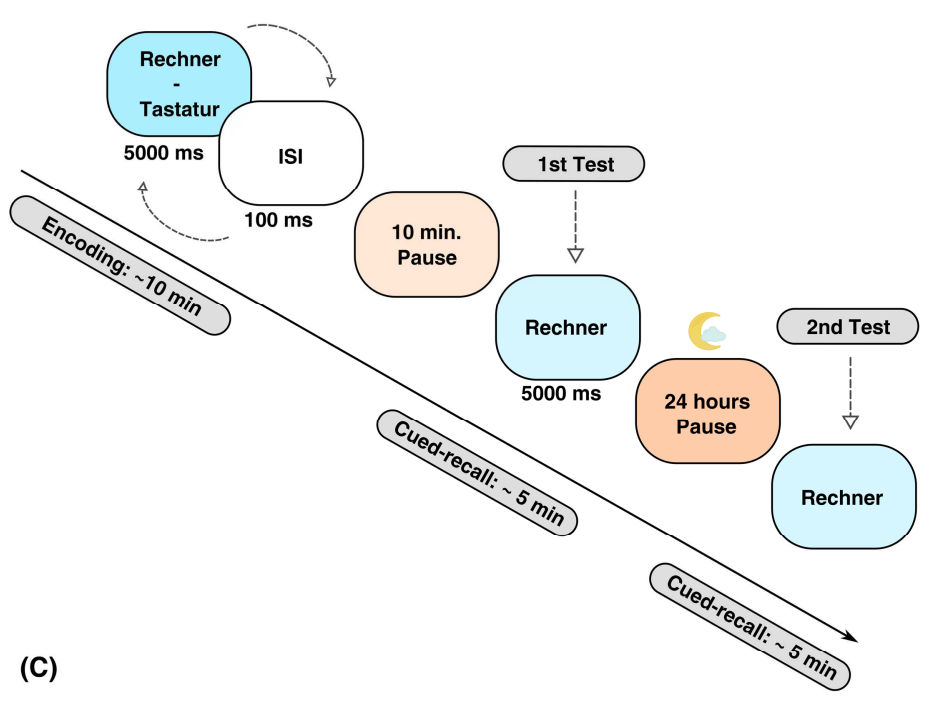

(B)

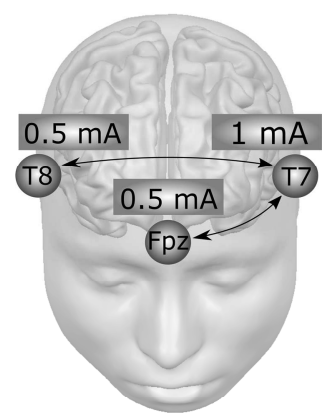

(D)

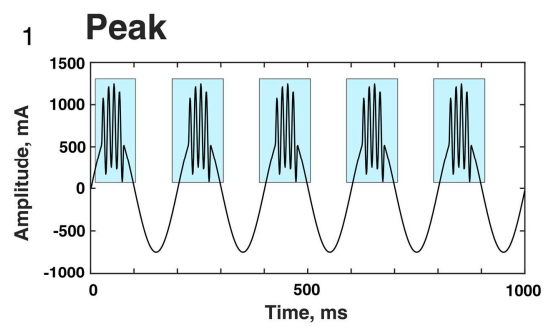

2 Trough

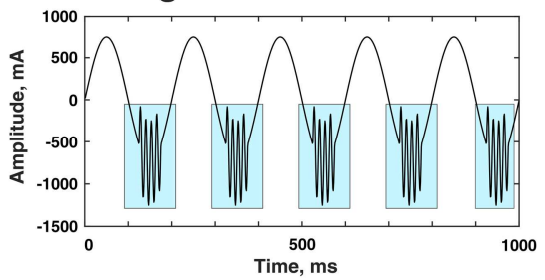

3 Continuous

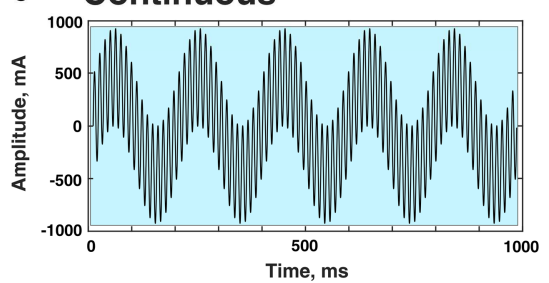


(A)

First cued-recall

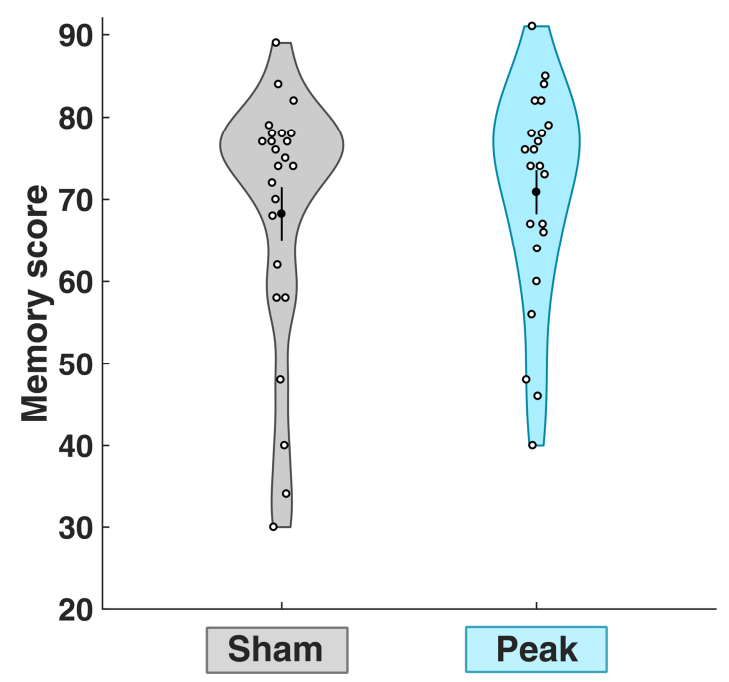

(B)

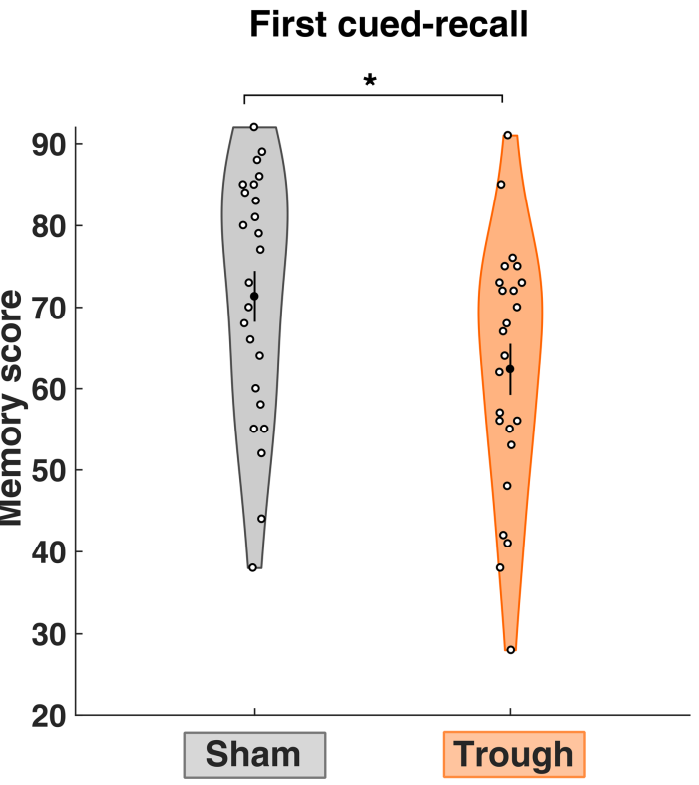

(C)

First cued-recall

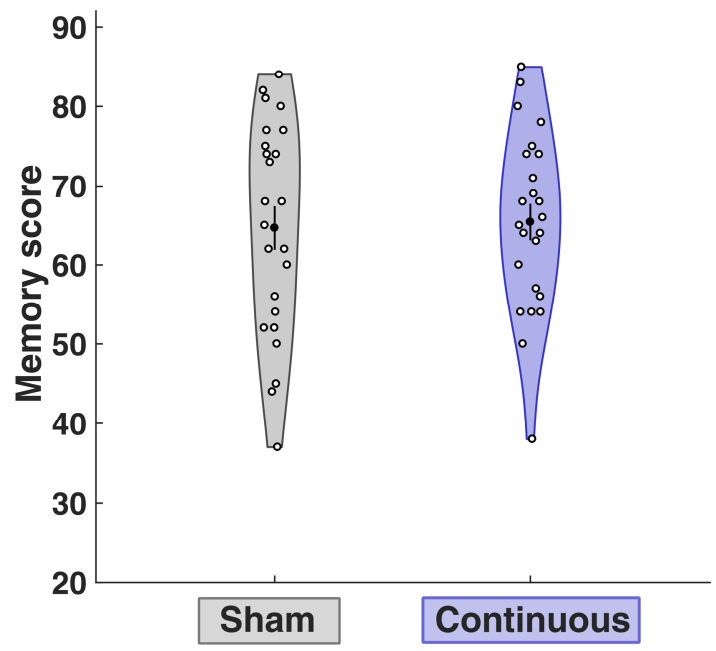

Second cued-recall

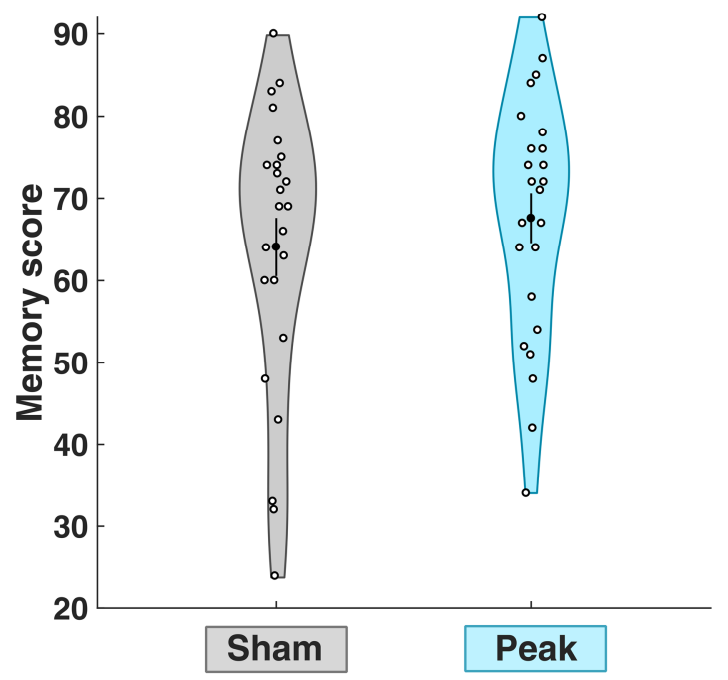

Second cued-recall

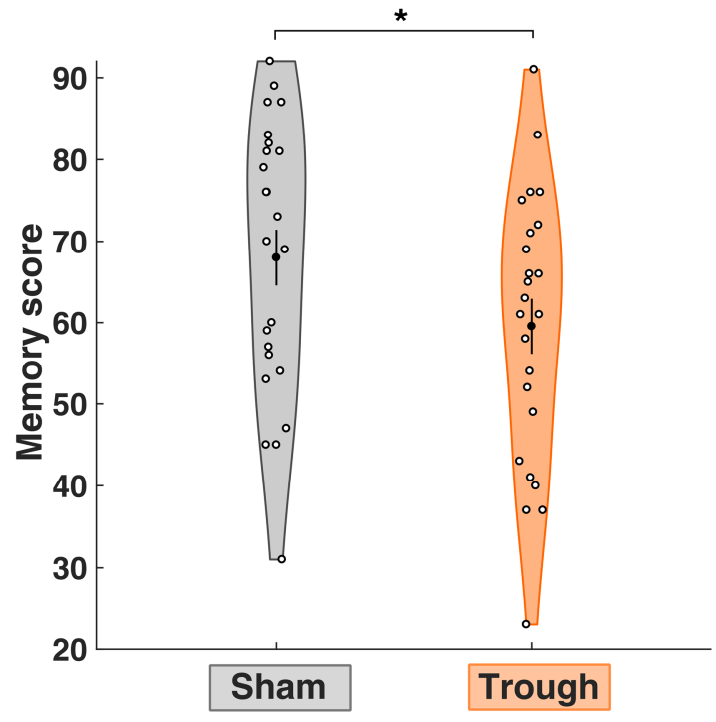

Second cued-recall

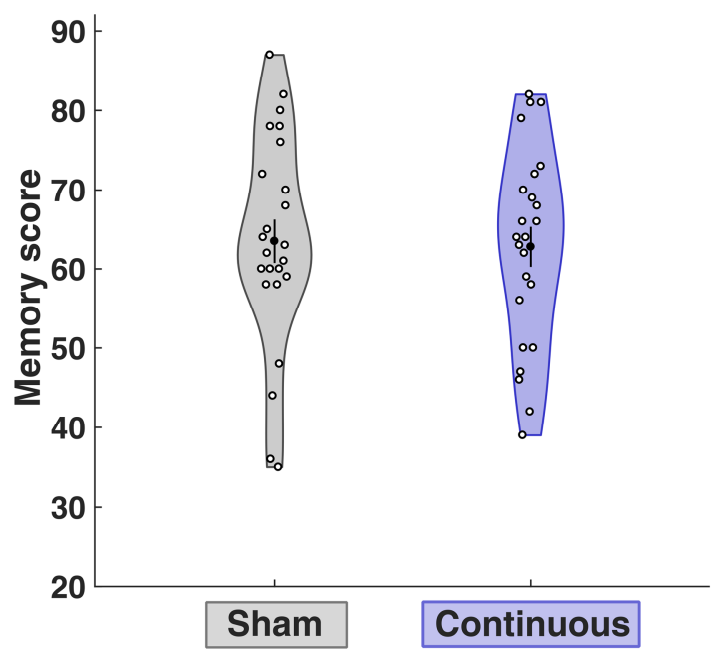




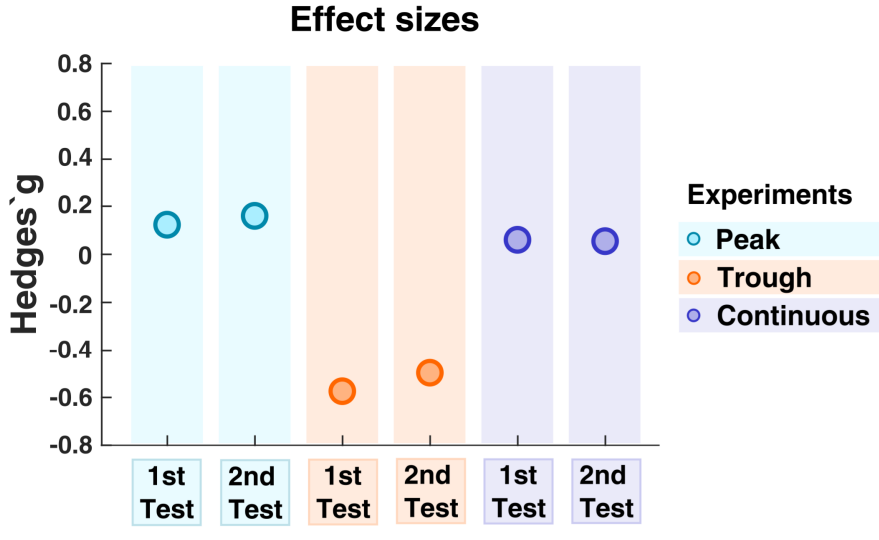


(A)

Peak $(n=24)$

First cued-recall

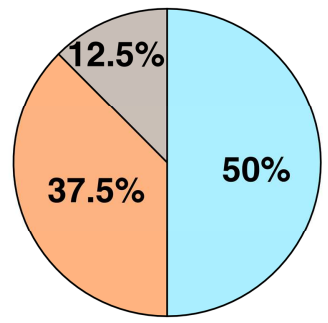

Second cued-recall

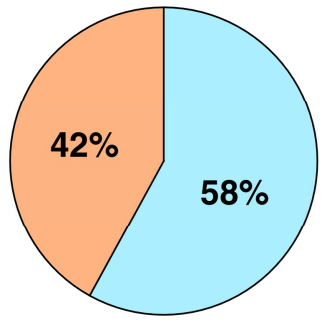

Performance change

Oincrease

decrease

same

(B)

Trough $(n=24)$

First cued-recall

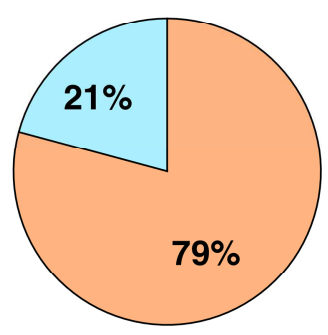

Second cued-recall

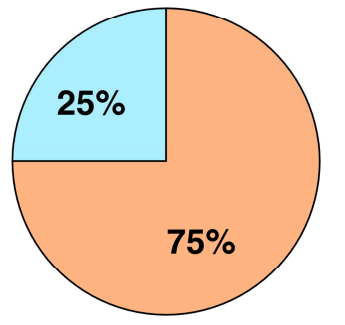

increase

decrease

same

(C)

$$
\text { Continuous }(n=24)
$$

First cued-recall

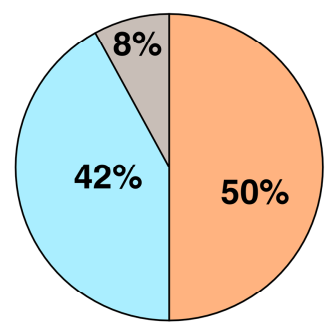

Second cued-recall

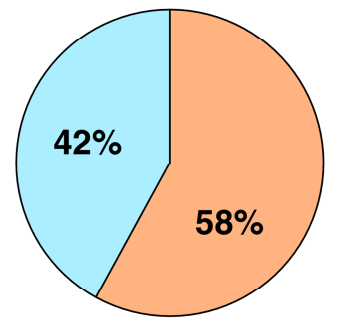

increase

decrease same 


\section{Highlights:}

- Theta-gamma cross-frequency tACS can modulate verbal declarative memory encoding

- Gamma bursts coupled to the trough of theta wave hinder memory performance

- Evidence is added for the involvement of theta-gamma coupling in memory formation 


\subsection{Supplementary material}

\section{Task}

The full dataset was additionally analyzed by using an alternative scoring criterion. In this second analysis approach, morphological differences such as plural/singular exchanges were neglected (Bacharach, 1972; Koppenaal \& Glanzer, 1990), and participants received a full score for a recalled word. Words recalled outside of the cued-recall's 5000 milliseconds time window (i.e., late answers) were treated as misses (Tulving \& Arbuckle, 1963) and no points were added. This different approach led to the same statistical results on the behavioral performance as the first analysis approach. The dependent variable (performance) was compared between conditions with the non-parametric Wilcoxon signed-ranks test and p-values were Bonferroni corrected for multiple comparisons.

For the first experiment (TGp-tACS), memory performance was not significantly different between the real tACS condition (70.6 \pm 14.9 mean score) and active sham condition $(68.3 \pm 15.6$ mean score) either for the first cued-recall $(\mathrm{Z}=1.293, \mathrm{p}=.392)$. No significant difference was found between the real tACS condition $(66.7 \pm 16.2$ mean score) and active sham stimulation condition $(64 \pm 17.3$ mean score $)$ on the second cued-recall $(Z=1.219, p=.446)$. In the second experiment (TGt-tACS), a significant impairment in performance occurred between real tACS $(62.5 \pm 15.5$ mean score $)$ and active sham stimulation (71.6 \pm mean score $)$ on the first cued-recall $(Z=-2.976, p=.006)$. This effect was also observed on the second cued-recall $(Z=-2.816, p=$ $.010)$, where real tACS (59.9 \pm 16.2 mean score) led to a significant decrease in memory performance compared to active sham stimulation $(68.3 \pm 16.6$ mean score). For the third experiment (TGc-tACS), there was no significant difference $(Z=-.046, p=1.0)$ between real 
tACS $(65.5 \pm 11.4$ mean score $)$ and the active sham stimulation $(65.1 \pm 13.3$ mean score $)$ on the first cued-recall, or between real tACS $(62.7 \pm 12.2$ mean score $)$ and the active sham condition $(63.9 \pm 13.4$ mean score $)$ on the second cued-recall $(Z=-.632, p=1.0)$ 


\section{Summary and concluding remarks}

The work conducted for this doctoral thesis is sustained on the premise that brain activity can be externally influenced by weak electrical currents. These currents can cross the barrier of several anatomical compartments and reach an extremely folded surface, where millions of neurons align themselves in different directions. Besides, the brain cells are also highly intertwined by complex layers of synaptic physical approximations and electrochemical cascades, which have to calibrate their activity in space and time. This activity is paced in a seesaw influx of excitation and inhibition, resulting in ever going cycles that rise as detectable brain rhythms. This activity is also susceptible for internal perturbations, which can translate into pathological states. The quest of the projects described in this work was to find possible ways of externally improve episodic memory performance in the healthy brain, with the ultimate goal as helping future research for clinical populations.

The assumptions' framework brought together to form this thesis were the following: (1) the several cortical nodes composing the verbal episodic memory network are susceptible to external perturbations, and, by that, functional changes can be induced; (2) NIBS are techniques that can be used to establish causal brain-behavior relations (Polanía et al., 2018), and its use for modulating functions is effective and reliable; (3) anodal tDCS can enhance neuronal excitability by depolarizing neuronal membrane, and also can decrease GABA levels at the targeted sites (Stagg et al., 2018), being a potentially strong paradigm to memory encoding augmentation; (4) naturally occurring theta-gamma phase-amplitude at temporal lobe structures is a potential target for tACS, its functional relation to verbal episodic memory can be tested by tES, and possibly, memory improvements can be tACS-induced. 
In the first study, where the left pre-frontal cortex was targeted with anodal tDCS, we chose to stimulate during encoding or recall (as a control group) with $1 \mathrm{~mA}$ current during 20 minutes. Contrary to our expectations and hypotheses, we could not observe a significant improvement in memory performance, given the chosen stimulation parameters. Still, our results are not distant from obtained with $1 \mathrm{~mA}$ tDCS in memory encoding paradigms so far, where mixed results and small effect sizes were the general finding (Dedoncker et al., 2016a; Manenti et al., 2013, 2012). Noteworthy is, that our results do not disprove the efficacy or potential of tDCS for episodic memory augmentation, but brings the necessity of uncovering better stimulation parameters for it. Factors as current intensity, stimulation duration and target site are first-order candidates for further experimental projects. Regarding stimulation duration, recently data demonstrated that the standard 20 minutes stimulation in motor cortex paradigms may not be directly translatable to the cognitive domain (Parkin et al., 2018). One possible successful use of tDCS in memory improvement can be the combination with an additional intervention, as it showed beneficial effects when combined with memory training and serotonergic enhancement (Antonenko et al., 2018; Prehn et al., 2017).

In the second study, we were the first research group to apply cross-frequency tACS to modulate episodic memory. Here, we targeted the left temporal cortex, choosing to stimulate with $1 \mathrm{~mA}$ current for 10 minutes during encoding of verbal content. Using a multi-electrode montage based on a computational model optimization, we measured a large sample of healthy participants and were able to significantly impair memory encoding in one of the experimental groups. Contrary to our hypothesis, when gamma was coupled to the peak of the theta carrying frequency, we found no improvement in memory performance. Nevertheless, we add evidence to the causal participation of theta-gamma cross-frequency coupling to episodic memory encoding. Additional 
online electrophysiology recordings might have brought a more definitive answer to which extent cross-frequency coupling was manipulated by tACS, although online recordings in tES are technically limited due to artifacts, and their current reliability a matter of strong debate (Neuling, Ruhnau, Weisz, Herrmann, \& Demarchi, 2017; Noury \& Siegel, 2018). Also, further research has to be conducted on the waveform action when gamma is coupled to the trough of the theta cycle, to possibly diverse memory tasks and different stimulation parameters (higher intensities and alternative electrode montages).

Notwithstanding, difficulties in finding robust parameters to augment human episodic memory non-invasively are being tackled by a different line of action, meaning, deep brain stimulation. These acute stimulation paradigms, usually delivered from electrocorticography $(\mathrm{ECoG})$ or depth electrodes, normally profit on pre-surgical electrophysiological measurements on epileptic patients to conduct studies or trials (K. Kim et al., 2016). By targeting neocortical areas or medial temporal lobe structures, usually with low or high gamma frequencies (e.g. 50, 100 or $200 \mathrm{~Hz}$ ), these stimulation protocols aim to mimic the normal physiological oscillatory patterns of episodic memory encoding (Lee, Fell, \& Axmacher, 2013), and bring maximum focality stimulating the memory nodes on site. Curiously, in verbal memory, the majority of the studies were able to disrupt memory encoding, but not improve it (Jacobs et al., 2016; Lee et al., 2013; Merkow et al., 2017). Possible solutions to the impairments can come with better synchronization of the stimulating waves with the normal physiology, using machine learning paradigms to look at the optimal or sub-optimal brain states and stimulate according to them (Ezzyat et al., 2017; Fell et al., 2013; Kucewicz et al., 2018). Nonetheless, invasive techniques also do not come with limitations: they are mainly restricted to non-healthy subjects' cohorts, and to the stimulation sites with clinical relevance. Still, focal interventions as invasive stimulation 
bring the promise of revealing further insights in memory machinery and on effective interventions to benefit memory impairments in patients.

From the non-invasive frontier, it is salutary to discuss some limitations of the studies conducted for this thesis. In a broader term, combining NIBS with other research methods would bring a clearer picture and more conclusive derivations of what was driven or not by the applied electrical currents (Polanía et al., 2018). Targeting can be also a debatable issue in the first study, due to the lack of spatial precision of tES, in comparison to other NIBS interventions. However, the use of neuronavigation may have affected the outcomes. The dosing employed is also a parameter that can highly impact the outcome of our studies, since the electric fields magnitude generated inside the skull by $1 \mathrm{~mA}$ tES can fall below or be in the limit threshold for modulating neuronal activity (Datta et al., 2009; Miranda, Mekonnen, Salvador, \& Ruffini, 2013; Opitz et al., 2016). That said, a linear increase in the dosing may not lead to linear outcomes, (Batsikadze, Moliadze, Paulus, Kuo, \& Nitsche, 2013; Parkin et al., 2018), and careful optimal intensities for different cognitive modalities have to be investigated. Electrode placement can rise now as another source of strong variation in studies relying on computational models to estimate the tES-generated electric fields. A recent study using intracranial recordings in human patients showed that fields predicted by models can vary immensely from real data when electrode placement diverge more than $1 \mathrm{~cm}$ between them (Opitz et al., 2018). This has large implications for model-driven tES protocols and for repeated-measures design as in the studies of this thesis.

Concluding, this research demonstrated that tES can be a tool to modulate episodic memory in a meaningful way. The large myriad of methodological parameters that can be explored in such investigations require optimizations coming from several technical advances in neurosciences, such as timelier precise stimulation tools, close loop systems, multi-electrode solutions, 
investigation of higher current intensities, and, in the case of episodic memory, the simultaneous stimulation of several nodes of the cortical memory network. Additionally, the experimentation with interfering alternating currents (Grossman et al., 2017) in the $\mathrm{kHz}$ range is a promising frontier for memory interventions, if the feasibility of targeting deep brain structures (for memory, the hippocampal formation) with non-invasive currents turn out to be executable and targets can be accurately reached. 


\section{Bibliography}

Alekseichuk, I., Turi, Z., Amador de Lara, G., Antal, A., \& Paulus, W. (2016). Spatial Working Memory in Humans Depends on Theta and High Gamma Synchronization in the Prefrontal Cortex. Current Biology, 26(12), 1513-1521. https://doi.org/10.1016/j.cub.2016.04.035

Ali, M. M., Sellers, K. K., \& Frohlich, F. (2013). Transcranial Alternating Current Stimulation Modulates Large-Scale Cortical Network Activity by Network Resonance. Journal of Neuroscience, 33(27), 11262-11275. https://doi.org/10.1523/JNEUROSCI.5867-12.2013

Amadi, U., Ilie, A., Johansen-Berg, H., \& Stagg, C. J. (2014). Polarity-specific effects of motor transcranial direct current stimulation on fMRI resting state networks. NeuroImage, 88 , 155-161. https://doi.org/10.1016/j.neuroimage.2013.11.037

Ambrus, G. G., Pisoni, A., Primaßin, A., Turi, Z., Paulus, W., \& Antal, A. (2015). Bi-frontal transcranial alternating current stimulation in the ripple range reduced overnight forgetting. Frontiers in Cellular Neuroscience, 9(September), 374. https://doi.org/10.3389/fncel.2015.00374

Antal, A., Alekseichuk, I., Bikson, M., Brockmöller, J., Brunoni, A. R., Chen, R., ... Paulus, W. (2017). Low intensity transcranial electric stimulation: Safety, ethical, legal regulatory and application guidelines. Clinical Neurophysiology, 128(9), 1774-1809. https://doi.org/10.1016/j.clinph.2017.06.001

Antal, A., Boros, K., Poreisz, C., Chaieb, L., Terney, D., \& Paulus, W. (2008). Comparatively weak after-effects of transcranial alternating current stimulation (tACS) on cortical excitability in humans. Brain Stimulation, 1(2), 97-105.

https://doi.org/10.1016/j.brs.2007.10.001

Antal, A., \& Herrmann, C. S. (2016). Transcranial Alternating Current and Random Noise Stimulation: Possible Mechanisms. Neural Plasticity, 2016, 1-12. https://doi.org/10.1155/2016/3616807

Antal, A., Terney, D., Poreisz, C., \& Paulus, W. (2007). Towards unravelling task-related modulations of neuroplastic changes induced in the human motor cortex. European Journal of Neuroscience, 26(9), 2687-2691. https://doi.org/10.1111/j.1460-9568.2007.05896.x

Antonenko, D., Külzow, N., Sousa, A., Prehn, K., Grittner, U., \& Flöel, A. (2018). Neurobiology of Aging Neuronal and behavioral effects of multi-day brain stimulation and memory training. Neurobiology of Aging, 61, 245-254. https://doi.org/10.1016/j.neurobiolaging.2017.09.017

Axmacher, N., Elger, C. E., \& Fell, J. (2009). The specific contribution of neuroimaging versus neurophysiological data to understanding cognition. Behavioural Brain Research, 200(1), 1-6. https://doi.org/10.1016/j.bbr.2009.01.028

Bacharach, V. R. (1972). Semantic and syntactic constraints on free-recall learning of sentential material. Journal of Experimental Psychology, 96(1), 223-225. 
https://doi.org/10.1037/h0033476

Batsikadze, G., Moliadze, V., Paulus, W., Kuo, M.-F., \& Nitsche, M. a. (2013). Partially nonlinear stimulation intensity-dependent effects of direct current stimulation on motor cortex excitability in humans. The Journal of Physiology, 591, 1987-2000.

https://doi.org/10.1113/jphysiol.2012.249730

Bergmann, T. O., Karabanov, A., Hartwigsen, G., Thielscher, A., \& Siebner, H. R. (2016). Combining non-invasive transcranial brain stimulation with neuroimaging and electrophysiology: Current approaches and future perspectives. NeuroImage, 140(February), 4-19. https://doi.org/10.1016/j.neuroimage.2016.02.012

Bikson, M., Brunoni, A. R., Charvet, L. E., Clark, V. P., Cohen, L. G., Deng, Z.-D., ... Lisanby, S. H. (2018). Rigor and reproducibility in research with transcranial electrical stimulation: An NIMH-sponsored workshop. Brain Stimulation, 11(3), 465-480. https://doi.org/10.1016/j.brs.2017.12.008

Bikson, M., Inoue, M., Akiyama, H., Deans, J. K., Fox, J. E., Miyakawa, H., \& Jefferys, J. G. R. (2004). Effects of uniform extracellular DC electric fields on excitability in rat hippocampal slices in vitro. The Journal of Physiology, 557(1), 175-190. https://doi.org/10.1113/jphysiol.2003.055772

Braun, V., Sokoliuk, R., \& Hanslmayr, S. (2017). On the effectiveness of event-related beta tACS on episodic memory formation and motor cortex excitability. Brain Stimulation, 10(5), 910-918. https://doi.org/10.1016/j.brs.2017.04.129

Canolty, R. T., \& Knight, R. T. (2010). The functional role of cross-frequncy coupling. Trends in Cognitive Sciences, 14(11), 506-515. https://doi.org/10.1016/j.tics.2010.09.001.The

Chew, T., Ho, K.-A., \& Loo, C. K. (2015). Inter- and Intra-individual Variability in Response to Transcranial Direct Current Stimulation (tDCS) at Varying Current Intensities. Brain Stimulation, 1-8. https://doi.org/10.1016/j.brs.2015.07.031

Colgin, L. L. (2015). Theta-gamma coupling in the entorhinal-hippocampal system. Current Opinion in Neurobiology, 31, 45-50. https://doi.org/10.1016/j.conb.2014.08.001

Datta, A., Bansal, V., Diaz, J., Patel, J., Reato, D., \& Bikson, M. (2009). Gyri-precise head model of transcranial direct current stimulation: Improved spatial focality using a ring electrode versus conventional rectangular pad. Brain Stimulation, 2(4), 201-207.e1. https://doi.org/10.1016/j.brs.2009.03.005

Davachi, L. (2006). Item, context and relational episodic encoding in humans. Current Opinion in Neurobiology, 16(6), 693-700. https://doi.org/10.1016/j.conb.2006.10.012

Deans, J. K., Powell, A. D., \& Jefferys, J. G. R. (2007). Sensitivity of coherent oscillations in rat hippocampus to AC electric fields. The Journal of Physiology, 583(Pt 2), 555-565. https://doi.org/10.1113/jphysiol.2007.137711

Dedoncker, J., Brunoni, A. R., Baeken, C., \& Vanderhasselt, M.-A. (2016a). A Systematic Review and Meta-Analysis of the Effects of Transcranial Direct Current Stimulation (tDCS) 
Over the Dorsolateral Prefrontal Cortex in Healthy and Neuropsychiatric Samples:

Influence of Stimulation Parameters. Brain Stimulation, 9(4), 1-17.

https://doi.org/10.1016/j.brs.2016.04.006

Dedoncker, J., Brunoni, A. R., Baeken, C., \& Vanderhasselt, M.-A. (2016b). The effect of the interval-between-sessions on prefrontal transcranial direct current stimulation (tdcs) on cognitive outcomes: A systematic review and meta-analysis. Journal of Neural Transmission, 123(10), No-Specified. https://doi.org/10.1016/j.brs.2016.04.006

Dickerson, B. C., \& Eichenbaum, H. (2010). The episodic memory system: neurocircuitry and disorders. Neuropsychopharmacology, 35(1), 86-104. https://doi.org/10.1038/npp.2009.126

Düzel, E., Penny, W. D., \& Burgess, N. (2010). Brain oscillations and memory. Current Opinion in Neurobiology, 20(2), 245-257. https://doi.org/10.1016/j.conb.2010.01.004

Eggert, T., Dorn, H., Sauter, C., Nitsche, M. A., Bajbouj, M., \& Danker-Hopfe, H. (2013). No Effects of Slow Oscillatory Transcranial Direct Current Stimulation (tDCS) on SleepDependent Memory Consolidation in Healthy Elderly Subjects. Brain Stimulation, 6(6), 938-945. https://doi.org/10.1016/j.brs.2013.05.006

Eichenbaum, H. (2017). Memory: Organization and Control. Annual Review of Psychology, 68(1), 19-45. https://doi.org/10.1146/annurev-psych-010416-044131

Emmerling, F., Martijn, C., Alberts, H. J. E. M., Thomson, A. C., David, B., Kessler, D., ... Sack, A. T. (2017). The ( non- ) replicability of regulatory resource depletion : A field report employing non- invasive brain stimulation. PLoS ONE, 12(3), 1-18. https://doi.org/10.6084/m9.figshare.4742944

Ezzyat, Y., Kragel, J. E., Burke, J. F., Levy, D. F., Lyalenko, A., Wanda, P., ... Kahana, M. J. (2017). Direct Brain Stimulation Modulates Encoding States and Memory Performance in Humans. Current Biology, 27, 1-8. https://doi.org/10.1016/j.cub.2017.03.028

Fell, J., Staresina, B. P., Do Lam, A. T. A., Widman, G., Helmstaedter, C., Elger, C. E., \& Axmacher, N. (2013). Memory Modulation by Weak Synchronous Deep Brain Stimulation: A Pilot Study. Brain Stimulation, 6(3), 270-273. https://doi.org/10.1016/j.brs.2012.08.001

Fertonani, A., \& Miniussi, C. (2017). Transcranial Electrical Stimulation. The Neuroscientist, 23(2), 109-123. https://doi.org/10.1177/1073858416631966

Fries, P. (2015). Rhythms for Cognition: Communication through Coherence. Neuron, 88(1), 220-235. https://doi.org/10.1016/j.neuron.2015.09.034

Fröhlich, F., \& McCormick, D. a. (2010). Endogenous electric fields may guide neocortical network activity. Neuron, 67(1), 129-143. https://doi.org/10.1016/j.neuron.2010.06.005

Fröhlich, F., \& Schmidt, S. L. (2013). Rational design of transcranial current stimulation (TCS) through mechanistic insights into cortical network dynamics. Frontiers in Human Neuroscience, 7(November), 1-5. https://doi.org/10.3389/fnhum.2013.00804

Fröhlich, F., Sellers, K. K., \& Cordle, A. L. (2015). Targeting the neurophysiology of cognitive 
systems with transcranial alternating current stimulation. Expert Review of Neurotherapeutics, 15(2), 145-167. https://doi.org/10.1586/14737175.2015.992782

Grossman, N., Bono, D., Dedic, N., Kodandaramaiah, S. B., Rudenko, A., Suk, H.-J., ... Boyden, E. S. (2017). Noninvasive Deep Brain Stimulation via Temporally Interfering Electric Fields. Cell, 169(6), 1029-1041.e16. https://doi.org/10.1016/j.cell.2017.05.024

Guerra, A., López-Alonso, V., Cheeran, B., \& Suppa, A. (2017). Solutions for managing variability in non-invasive brain stimulation studies. Neuroscience Letters, (December), 0 1. https://doi.org/10.1016/j.neulet.2017.12.060

Hannula, D. E., \& Ranganath, C. (2009). The Eyes Have It: Hippocampal Activity Predicts Expression of Memory in Eye Movements. Neuron, 63(5), 592-599. https://doi.org/10.1016/j.neuron.2009.08.025

Hanslmayr, S., Staresina, B. P., \& Bowman, H. (2016). Oscillations and Episodic Memory: Addressing the Synchronization/Desynchronization Conundrum. Trends in Neurosciences, 39(1), 16-25. https://doi.org/10.1016/j.tins.2015.11.004

Hanslmayr, S., \& Staudigl, T. (2014). How brain oscillations form memories - A processing based perspective on oscillatory subsequent memory effects. NeuroImage, 85, 648-655. https://doi.org/10.1016/j.neuroimage.2013.05.121

Helfrich, R. F., Schneider, T. R., Rach, S., Trautmann-Lengsfeld, S. A., Engel, A. K., \& Herrmann, C. S. (2014). Entrainment of Brain Oscillations by Transcranial Alternating Current Stimulation. Current Biology, 24(3), 333-339. https://doi.org/10.1016/j.cub.2013.12.041

Henke, K. (2010). A model for memory systems based on processing modes rather than consciousness. Nature Reviews. Neuroscience, 11(7), 523-32. https://doi.org/10.1038/nrn2850

Herrmann, C. S., Rach, S., Neuling, T., \& Strüber, D. (2013). Transcranial alternating current stimulation: a review of the underlying mechanisms and modulation of cognitive processes. Frontiers in Human Neuroscience, 7(June), 1-13. https://doi.org/10.3389/fnhum.2013.00279

Heusser, A. C., Poeppel, D., Ezzyat, Y., \& Davachi, L. (2016). Episodic sequence memory is supported by a theta-gamma phase code. Nature Neuroscience, 19(August), 1374-1383. https://doi.org/10.1038/nn.4374

Hill, A. T., Rogasch, N. C., Fitzgerald, P. B., \& Hoy, K. E. (2017). Effects of prefrontal bipolar and high-definition transcranial direct current stimulation on cortical reactivity and working memory in healthy adults. NeuroImage, 152(March), 142-157. https://doi.org/10.1016/j.neuroimage.2017.03.001

Holdefer, R. N., Sadleir, R., \& Russell, M. J. (2006). Predicted current densities in the brain during transcranial electrical stimulation. Clinical Neurophysiology, 117(6), 1388-1397. https://doi.org/10.1016/j.clinph.2006.02.020 
Horvath, J. C., Forte, J. D., \& Carter, O. (2014). Evidence that transcranial direct current stimulation (tDCS) generates little-to-no reliable neurophysiologic effect beyond MEP amplitude modulation in healthy human subjects_A systematic review. Neuropsychologia, 66, 213-236. https://doi.org/10.1016/j.neuropsychologia.2014.11.021

Horvath, J. C., Forte, J. D., \& Carter, O. (2015). Quantitative Review Finds No Evidence of Cognitive Effects in Healthy Populations From Single-session Transcranial Direct Current Stimulation (tDCS). Brain Stimulation, 8(3), 535-550. https://doi.org/10.1016/j.brs.2015.01.400

Huang, Y.-Z., Lu, M.-K., Antal, A., Classen, J., Nitsche, M., Ziemann, U., ... Rothwell, J. (2017). Plasticity induced by non-invasive transcranial brain stimulation: A position paper. Clinical Neurophysiology, 128(11), 2318-2329. https://doi.org/10.1016/j.clinph.2017.09.007

Huang, Y., Liu, A. A., Lafon, B., Friedman, D., Dayan, M., Wang, X., ... Parra, L. C. (2017). Measurements and models of electric fields in the in vivo human brain during transcranial electric stimulation. ELife, 6, e18834. https://doi.org/10.7554/eLife.18834

Hyafil, A., Giraud, A., Fontolan, L., \& Gutkin, B. (2015). Neural Cross-Frequency Coupling : Connecting Architectures, Mechanisms, and Functions. Trends in Neurosciences, 38(11), 725-740. https://doi.org/10.1016/j.tins.2015.09.001

Jacobs, J., Miller, J., Lee, S. A., Coffey, T., Watrous, A. J., Sperling, M. R., ... Rizzuto, D. S. (2016). Direct Electrical Stimulation of the Human Entorhinal Region and Hippocampus Impairs Memory. Neuron, 92(5), 983-990. https://doi.org/10.1016/j.neuron.2016.10.062

Javadi, A.-H., Glen, J. C., Halkiopoulos, S., Schulz, M., \& Spiers, H. J. (2017). Oscillatory Reinstatement Enhances Declarative Memory. The Journal of Neuroscience, 37(41), 99399944. https://doi.org/10.1523/JNEUROSCI.0265-17.2017

Javadi, A. H., \& Walsh, V. (2012). Transcranial direct current stimulation (tDCS) of the left dorsolateral prefrontal cortex modulates declarative memory. Brain Stimulation, 5(3), 231241. https://doi.org/10.1016/j.brs.2011.06.007

Jeong, W., Chung, C. K., \& Kim, J. S. (2015). Episodic memory in aspects of large-scale brain networks. Frontiers in Human Neuroscience, 9(August), 454.

https://doi.org/10.3389/fnhum.2015.00454

Jutras, M. J., \& Buffalo, E. a. (2010). Synchronous neural activity and memory formation. Current Opinion in Neurobiology, 20(2), 150-155. https://doi.org/10.1016/j.conb.2010.02.006

Kandel, E. R., Dudai, Y., \& Mayford, M. R. (2014). The molecular and systems biology of memory. Cell, 157(1), 163-186. https://doi.org/10.1016/j.cell.2014.03.001

Keeser, D., Meindl, T., Bor, J., Palm, U., Pogarell, O., Mulert, C., ... Padberg, F. (2011). Prefrontal Transcranial Direct Current Stimulation Changes Connectivity of Resting-State Networks during fMRI. Journal of Neuroscience, 31(43), 15284-15293. 
https://doi.org/10.1523/JNEUROSCI.0542-11.2011

Kim, H. (2011). Neural activity that predicts subsequent memory and forgetting: A meta-analysis of 74 fMRI studies. NeuroImage, 54(3), 2446-2461. https://doi.org/10.1016/j.neuroimage.2010.09.045

Kim, K., Ekstrom, A. D., \& Tandon, N. (2016). A network approach for modulating memory processes via direct and indirect brain stimulation: Toward a causal approach for the neural basis of memory. Neurobiology of Learning and Memory, 134(Part A), 162-177. https://doi.org/10.1016/j.nlm.2016.04.001

Kirov, R., Weiss, C., Siebner, H. R., Born, J., \& Marshall, L. (2009). Slow oscillation electrical brain stimulation during waking promotes EEG theta activity and memory encoding. Proceedings of the National Academy of Sciences of the United States of America, 106(36), 15460-15465. https://doi.org/10.1073/pnas.0904438106

Koppenaal, L., \& Glanzer, M. (1990). An examination of the continuous distractor task and the "long-term recency effect." Memory \& Cognition, 18(2), 183-195. https://doi.org/10.3758/BF03197094

Krause, M. R., Zanos, T. P., Csorba, B. A., Pilly, P. K., Choe, J., Phillips, M. E., ... Pack, C. C. (2017). Transcranial Direct Current Stimulation Facilitates Associative Learning and Alters Functional Connectivity in the Primate Brain. Current Biology, 27(20), 3086-3096.e3. https://doi.org/10.1016/j.cub.2017.09.020

Kucewicz, M. T., Berry, B. M., Kremen, V., Miller, L. R., Khadjevand, F., Ezzyat, Y., ... Worrell, G. A. (2018). Electrical Stimulation Modulates High $\gamma$ Activity and Human Memory Performance. Eneuro, 5(1), ENEURO.0369-17.2018. https://doi.org/10.1523/ENEURO.0369-17.2018

Kühn, S., \& Gallinat, J. (2014). Segregating cognitive functions within hippocampal formation: a quantitative meta-analysis on spatial navigation and episodic memory. Human Brain Mapping, 35(4), 1129-42. https://doi.org/10.1002/hbm.22239

Kuo, M.-F., Grosch, J., Fregni, F., Paulus, W., \& Nitsche, M. A. (2007). Focusing Effect of Acetylcholine on Neuroplasticity in the Human Motor Cortex. Journal of Neuroscience, 27(52), 14442-14447. https://doi.org/10.1523/JNEUROSCI.4104-07.2007

Kuo, M.-F., Paulus, W., \& Nitsche, M. A. (2008). Boosting Focally-Induced Brain Plasticity by Dopamine. Cerebral Cortex, 18(3), 648-651. https://doi.org/10.1093/cercor/bhm098

Ladenbauer, J., Külzow, N., Passmann, S., Antonenko, D., Grittner, U., Tamm, S., \& Flöel, A. (2016). NeuroImage Brain stimulation during an afternoon nap boosts slow oscillatory activity and memory consolidation in older adults. NeuroImage, 142, 311-323. https://doi.org/10.1016/j.neuroimage.2016.06.057

Ladenbauer, X. J., Ladenbauer, X. J., Ku, N., Boor, R. De, Avramova, E., Grittner, X. U., ... Theory, N. (2017). Promoting Sleep Oscillations and Their Functional Coupling by Transcranial Stimulation Enhances Memory Consolidation in Mild Cognitive Impairment. 
Journal of Neuroscience, 37(30), 7111-7124. https://doi.org/10.1523/JNEUROSCI.026017.2017

Lara, G. A. de, Alekseichuk, I., Turi, Z., Lehr, A., Antal, A., \& Paulus, W. (2018). Perturbation of theta-gamma coupling at the temporal lobe hinders verbal declarative memory. Brain Stimulation, 11(3), 509-517. https://doi.org/10.1016/j.brs.2017.12.007

Lara, G. A. de, Knechtges, P. N., Paulus, W., \& Antal, A. (2017). Anodal tDCS Over the Left DLPFC Did Not Affect the Encoding and Retrieval of Verbal Declarative Information. Frontiers in Neuroscience, 11(August), 1-9. https://doi.org/10.3389/fnins.2017.00452

Lee, H., Fell, J., \& Axmacher, N. (2013). Electrical engram: how deep brain stimulation affects memory. Trends in Cognitive Sciences, 17(11), 574-584. https://doi.org/10.1016/j.tics.2013.09.002

Lefaucheur, J.-P., Antal, A., Ayache, S. S., Benninger, D. H., Brunelin, J., Cogiamanian, F., ... Paulus, W. (2017). Evidence-based guidelines on the therapeutic use of transcranial direct current stimulation (tDCS). Clinical Neurophysiology, 128(1), 56-92. https://doi.org/10.1016/j.clinph.2016.10.087

Lega, B., Burke, J., Jacobs, J., \& Kahana, M. J. (2016). Slow-Theta-to-Gamma Phase-Amplitude Coupling in Human Hippocampus Supports the Formation of New Episodic Memories. Cerebral Cortex, 26(1), 268-278. https://doi.org/10.1093/cercor/bhu232

Li, L. M., Uehara, K., \& Hanakawa, T. (2015). The contribution of interindividual factors to variability of response in transcranial direct current stimulation studies. Frontiers in Cellular Neuroscience, 9(May). https://doi.org/10.3389/fncel.2015.00181

Liebetanz, D., Koch, R., Mayenfels, S., König, F., Paulus, W., \& Nitsche, M. A. (2009). Safety limits of cathodal transcranial direct current stimulation in rats. Clinical Neurophysiology, 120(6), 1161-1167. https://doi.org/10.1016/j.clinph.2009.01.022

Lisman, J. E., \& Jensen, O. (2013). The Theta-Gamma Neural Code. Neuron, 77(6), 1002-1016. https://doi.org/10.1016/j.neuron.2013.03.007

Manenti, R., Brambilla, M., Petesi, M., Ferrari, C., \& Cotelli, M. (2013). Enhancing verbal episodic memory in older and young subjects after non-invasive brain stimulation. Frontiers in Aging Neuroscience, 5(SEP), 1-9. https://doi.org/10.3389/fnagi.2013.00049

Manenti, R., Cotelli, M., Robertson, I. H., \& Miniussi, C. (2012). Transcranial brain stimulation studies of episodic memory in young adults, elderly adults and individuals with memory dysfunction: A review. Brain Stimulation, 5(2), 103-109. https://doi.org/10.1016/j.brs.2012.03.004

Manuel, A. L., \& Schnider, A. (2016). Effect of prefrontal and parietal tDCS on learning and recognition of verbal and non-verbal material. Clinical Neurophysiology, 127(7), 25922598. https://doi.org/10.1016/j.clinph.2016.04.015

Marshall, L., Helgadóttir, H., Mölle, M., \& Born, J. (2006). Boosting slow oscillations during sleep potentiates memory. Nature, 444(7119), 610-613. 
https://doi.org/10.1038/nature05278

Marshall, L., Kirov, R., Brade, J., Mölle, M., \& Born, J. (2011). Transcranial Electrical Currents to Probe EEG Brain Rhythms and Memory Consolidation during Sleep in Humans. PLoS ONE, 6(2), e16905. https://doi.org/10.1371/journal.pone.0016905

Medvedeva, A., Materassi, M., Neacsu, V., Beresford-Webb, J., Hussin, A., Khan, N., ... Galli, G. (2018). Effects of Anodal Transcranial Direct Current Stimulation Over the Ventrolateral Prefrontal Cortex on Episodic Memory Formation and Retrieval. Cerebral Cortex, (January), 1-9. https://doi.org/10.1093/cercor/bhx347

Merkow, M. B., Burke, J. F., Ramayya, A. G., Sharan, A. D., Sperling, M. R., \& Kahana, M. J. (2017). Stimulation of the human medial temporal lobe between learning and recall selectively enhances forgetting. Brain Stimulation, 10(3), 645-650. https://doi.org/10.1016/j.brs.2016.12.011

Miniussi, C., Brignani, D., \& Pellicciari, M. C. (2012). Combining Transcranial Electrical Stimulation With Electroencephalography. Clinical EEG and Neuroscience, 43(3), 184 191. https://doi.org/10.1177/1550059412444976

Miniussi, C., Harris, J. A., \& Ruzzoli, M. (2013). Modelling non-invasive brain stimulation in cognitive neuroscience. Neuroscience \& Biobehavioral Reviews, 37(8), 1702-1712. https://doi.org/10.1016/j.neubiorev.2013.06.014

Miranda, P. C., Mekonnen, A., Salvador, R., \& Ruffini, G. (2013). The electric field in the cortex during transcranial current stimulation. NeuroImage, 70, 48-58. https://doi.org/10.1016/j.neuroimage.2012.12.034

Moliadze, V., Antal, A., \& Paulus, W. (2010). Boosting brain excitability by transcranial high frequency stimulation in the ripple range. The Journal of Physiology, 588(24), 4891-4904. https://doi.org/10.1113/jphysiol.2010.196998

Moliadze, V., Atalay, D., Antal, A., \& Paulus, W. (2012). Close to threshold transcranial electrical stimulation preferentially activates inhibitory networks before switching to excitation with higher intensities. Brain Stimulation, 5(4), 505-511. https://doi.org/10.1016/j.brs.2011.11.004

Morris, R. (2013). Neurobiology of Learning and Memory. In D. W. Pfaff (Ed.), Neuroscience in the 21 st Century. From Basic to Clinical (pp. 2173-2212). New York, NY: Springer New York. https://doi.org/10.1007/978-1-4614-1997-6

Neuling, T., Ruhnau, P., Weisz, N., Herrmann, C. S., \& Demarchi, G. (2017). Faith and oscillations recovered: On analyzing EEG/MEG signals during tACS. Neurolmage, 147, 960-963. https://doi.org/10.1016/j.neuroimage.2016.11.022

Nitsche, M. A., Cohen, L. G., Wassermann, E. M., Priori, A., Lang, N., Antal, A., .. PascualLeone, A. (2008). Transcranial direct current stimulation: State of the art 2008. Brain Stimulation, 1, 206-223. https://doi.org/10.1016/j.brs.2008.06.004

Nitsche, M. A., Kuo, M.-F., Karrasch, R., Wächter, B., Liebetanz, D., \& Paulus, W. (2009). 
Serotonin Affects Transcranial Direct Current-Induced Neuroplasticity in Humans. Biological Psychiatry, 66(5), 503-508. https://doi.org/10.1016/j.biopsych.2009.03.022

Nitsche, M. A., Lampe, C., Antal, A., Liebetanz, D., Lang, N., Tergau, F., \& Paulus, W. (2006). Dopaminergic modulation of long-lasting direct current-induced cortical excitability changes in the human motor cortex. European Journal of Neuroscience, 23(January), 16511657. https://doi.org/10.1111/j.1460-9568.2006.04676.x

Nitsche, M. A., Müller-Dahlhaus, F., Paulus, W., \& Ziemann, U. (2012). The pharmacology of neuroplasticity induced by non-invasive brain stimulation: building models for the clinical use of CNS active drugs. The Journal of Physiology, 590(19), 4641-4662. https://doi.org/10.1113/jphysiol.2012.232975

Nitsche, M. A., \& Paulus, W. (2000). Excitability changes induced in the human motor cortex by weak transcranial direct current stimulation. The Journal of Physiology, 527 Pt 3, 633-9. https://doi.org/PHY_1055 [pii]

Nitsche, M. A., \& Paulus, W. (2001). Sustained excitability elevations induced by transcranial DC motor cortex stimulation in humans. Neurology, 57(10), 1899-1901. https://doi.org/10.1212/WNL.57.10.1899

Nitsche, M. A., \& Paulus, W. (2011). Transcranial direct current stimulation - Update 2011. Restorative Neurology and Neuroscience, 29, 463-492. https://doi.org/10.3233/RNN-20110618

Notturno, F., Marzetti, L., Pizzella, V., Uncini, A., \& Zappasodi, F. (2014). Local and remote effects of transcranial direct current stimulation on the electrical activity of the motor cortical network. Human Brain Mapping, 35(5), 2220-2232. https://doi.org/10.1002/hbm.22322

Noury, N., \& Siegel, M. (2018). Analyzing EEG and MEG signals recorded during tES, a reply. NeuroImage, 167, 53-61. https://doi.org/10.1016/j.neuroimage.2017.11.023

Ofen, N., Kao, Y., Sokol-Hessner, P., Kim, H., Whitfield-Gabrieli, S., \& Gabrieli, J. D. E. (2007). Development of the declarative memory system in the human brain. Nature Neuroscience, 10(9), 1198-205. https://doi.org/10.1038/nn1950

Opitz, A., Falchier, A., Yan, C., Yeagle, E., \& Linn, G. (2016). Spatiotemporal structure of intracranial electric fields induced by transcranial electric stimulation in human and nonhuman primates. Scientific Reports, 6(August), 1-11. https://doi.org/10.1101/053892

Opitz, A., Paulus, W., Will, A., \& Thielscher, A. (2015). Anatomical determinants of the electric field during transcranial direct current stimulation. NeuroImage, 109, 2. https://doi.org/10.1016/j.neuroimage.2015.01.033

Opitz, A., Windhoff, M., Heidemann, R. M., Turner, R., \& Thielscher, A. (2011). How the brain tissue shapes the electric field induced by transcranial magnetic stimulation. NeuroImage, 58(3), 849-859. https://doi.org/10.1016/j.neuroimage.2011.06.069

Opitz, A., Yeagle, E., Thielscher, A., Schroeder, C., Mehta, A. D., \& Milham, M. P. (2018). On 
the importance of precise electrode placement for targeted transcranial electric stimulation. NeuroImage, 181(July), 560-567. https://doi.org/10.1016/j.neuroimage.2018.07.027

Ozen, S., Sirota, A., Belluscio, M. a, Anastassiou, C. a, Stark, E., Koch, C., \& Buzsaki, G. (2010). Transcranial Electric Stimulation Entrains Cortical Neuronal Populations in Rats. Journal of Neuroscience, 30(34), 11476-11485. https://doi.org/10.1523/JNEUROSCI.525209.2010

Parkin, B. L., Bhandari, M., Glen, J. C., \& Walsh, V. (2018). The physiological effects of transcranial electrical stimulation do not apply to parameters commonly used in studies of cognitive neuromodulation. Neuropsychologia. https://doi.org/10.1016/j.neuropsychologia.2018.03.030

Parkin, B. L., Ekhtiari, H., \& Walsh, V. F. (2015). Non-invasive Human Brain Stimulation in Cognitive Neuroscience: A Primer. Neuron, 87(5), 932-945. https://doi.org/10.1016/j.neuron.2015.07.032

Paßmann, S., Külzow, N., Ladenbauer, J., Antonenko, D., Grittner, U., Tamm, S., \& Flöel, A. (2016). Boosting Slow Oscillatory Activity Using tDCS during Early Nocturnal Slow Wave Sleep Does Not Improve Memory Consolidation in Healthy Older Adults. Brain Stimulation, 9(5), 730-739. https://doi.org/10.1016/j.brs.2016.04.016

Paulus, W. (2011). Transcranial electrical stimulation (tES , 21(5), 602-617. https://doi.org/10.1080/09602011.2011.557292

Peterchev, A. V., Wagner, T. a., Miranda, P. C., Nitsche, M. a., Paulus, W., Lisanby, S. H., ... Bikson, M. (2012). Fundamentals of transcranial electric and magnetic stimulation dose: Definition, selection, and reporting practices. Brain Stimulation, 5(4), 435-453. https://doi.org/10.1016/j.brs.2011.10.001

Pisoni, A., Turi, Z., Raithel, A., Ambrus, G. G., Alekseichuk, I., Schacht, A., ... Antal, A. (2015). Separating recognition processes of declarative memory via anodal tDCS: Boosting old item recognition by temporal and new item detection by parietal stimulation. PLoS ONE, 10(3), 1-17. https://doi.org/10.1371/journal.pone.0123085

Polanía, R., Nitsche, M. A., \& Ruff, C. C. (2018). Studying and modifying brain function with non-invasive brain stimulation. Nature Neuroscience. https://doi.org/10.1038/s41593-0170054-4

Poo, M., Pignatelli, M., Ryan, T. J., Tonegawa, S., Bonhoeffer, T., Martin, K. C., ... Stevens, C. (2016). What is memory? The present state of the engram. BMC Biology, 14(1), 40. https://doi.org/10.1186/s12915-016-0261-6

Prehn, K., Stengl, H., Grittner, U., Kosiolek, R., Ölschläger, A., Weidemann, A., \& Flöel, A. (2017). Effects of Anodal Transcranial Direct Current Stimulation and Serotonergic Enhancement on Memory Performance in Young and Older Adults. Neuropsychopharmacology, 42, 551-561. https://doi.org/10.1038/npp.2016.170

Preston, A. R., \& Eichenbaum, H. (2013). Interplay of Hippocampus and Prefrontal Cortex in 
Memory. Current Biology, 23(17), R764-R773. https://doi.org/10.1016/j.cub.2013.05.041

Priori, A., Berardelli, A., Rona, S., Accornero, N., \& Manfredi, M. (1998). Polarization of the human motor cortex through the scalp. NeuroReport, 9(10), 2257-2260. https://doi.org/10.1097/00001756-199807130-00020

Radman, T., Ramos, R. L., Brumberg, J. C., \& Bikson, M. (2009). Role of cortical cell type and morphology in subthreshold and suprathreshold uniform electric field stimulation in vitro. Brain Stimulation, 2(4), 215-228.e3. https://doi.org/10.1016/j.brs.2009.03.007

Radman, T., Su, Y., An, J. H., Parra, L. C., \& Bikson, M. (2007). Spike Timing Amplifies the Effect of Electric Fields on Neurons: Implications for Endogenous Field Effects. Journal of Neuroscience, 27(11), 3030-3036. https://doi.org/10.1523/JNEUROSCI.0095-07.2007

Raffin, E., \& Siebner, H. R. (2014). Transcranial brain stimulation to promote functional recovery after stroke. Current Opinion in Neurology, 27(1), 54-60. https://doi.org/10.1097/WCO.0000000000000059

Rahman, A., Reato, D., Arlotti, M., Gasca, F., Datta, A., Parra, L. C., \& Bikson, M. (2013). Cellular effects of acute direct current stimulation: somatic and synaptic terminal effects. The Journal of Physiology, 591(10), 2563-2578. https://doi.org/10.1113/jphysiol.2012.247171

Raichle, M. E. (2009). A brief history of human brain mapping. Trends in Neurosciences, 32(2), 118-126. https://doi.org/10.1016/j.tins.2008.11.001

Ranganath, C. (2010). A unified framework for the functional organization of the medial temporal lobes and the phenomenology of episodic memory. Hippocampus, 20(11), 12631290. https://doi.org/10.1002/hipo.20852

Reato, D., Rahman, A., Bikson, M., \& Parra, L. C. (2010). Low-Intensity Electrical Stimulation Affects Network Dynamics by Modulating Population Rate and Spike Timing. Journal of Neuroscience, 30(45), 15067-15079. https://doi.org/10.1523/JNEUROSCI.2059-10.2010

Reato, D., Rahman, A., Bikson, M., \& Parra, L. C. (2013). Effects of weak transcranial alternating current stimulation on brain activity - a review of known mechanisms from animal studies. Frontiers in Human Neuroscience, 7(October), 687. https://doi.org/10.3389/fnhum.2013.00687

Romei, V., Thut, G., \& Silvanto, J. (2016). Information-Based Approaches of Noninvasive Transcranial Brain Stimulation. Trends in Neurosciences, $x x$ (October), 1-14. https://doi.org/10.1016/j.tins.2016.09.001

Sahlem, G. L., Badran, B. W., Halford, J. J., Williams, N. R., Korte, J. E., Leslie, K., ... George, M. S. (2015). Oscillating Square Wave Transcranial Direct Current Stimulation (tDCS) Delivered During Slow Wave Sleep Does Not Improve Declarative Memory More Than Sham: A Randomized Sham Controlled Crossover Study. Brain Stimulation, 8(3), 528-534. https://doi.org/10.1016/j.brs.2015.01.414

Schott, B. H., Wüstenberg, T., Wimber, M., Fenker, D. B., Zierhut, K. C., Seidenbecher, C. I., ... 
Richardson-Klavehn, A. (2013). The relationship between level of processing and hippocampal-cortical functional connectivity during episodic memory formation in humans. Human Brain Mapping, 34(2), 407-424. https://doi.org/10.1002/hbm.21435

Sehm, B., Schafer, A., Kipping, J., Margulies, D., Conde, V., Taubert, M., ... Ragert, P. (2012). Dynamic modulation of intrinsic functional connectivity by transcranial direct current stimulation. Journal of Neurophysiology, 108(12), 3253-3263. https://doi.org/10.1152/jn.00606.2012

Soekadar, S. R., Witkowski, M., Cossio, E. G., Birbaumer, N., Robinson, S. E., \& Cohen, L. G. (2013). In vivo assessment of human brain oscillations during application of transcranial electric currents. Nature Communications, 4(May), 2032. https://doi.org/10.1038/ncomms3032

Stagg, C. J., Antal, A., \& Nitsche, M. A. (2018). Physiology of Transcranial Direct Current Stimulation. The Journal of ECT, OO(00), 1. https://doi.org/10.1097/YCT.0000000000000510

Stagg, C. J., \& Nitsche, M. a. (2011). Physiological Basis of Transcranial Direct Current Stimulation. The Neuroscientist, 17(1), 37-53. https://doi.org/10.1177/1073858410386614

Thielscher, A., Opitz, A., \& Windhoff, M. (2011). Impact of the gyral geometry on the electric field induced by transcranial magnetic stimulation. NeuroImage, 54(1), 234-243. https://doi.org/10.1016/j.neuroimage.2010.07.061

Thut, G., Miniussi, C., \& Gross, J. (2012). The functional importance of rhythmic activity in the brain. Current Biology, 22(16), R658-R663. https://doi.org/10.1016/j.cub.2012.06.061

Thut, G., Schyns, P. G., \& Gross, J. (2011). Entrainment of Perceptually Relevant Brain Oscillations by Non-Invasive Rhythmic Stimulation of the Human Brain. Frontiers in Psychology, 2(JUL), 1-10. https://doi.org/10.3389/fpsyg.2011.00170

To, W. T., De Ridder, D., Hart Jr., J., \& Vanneste, S. (2018). Changing Brain Networks Through Non-invasive Neuromodulation. Frontiers in Human Neuroscience, 12(April), 1-17. https://doi.org/10.3389/fnhum.2018.00128

Tonegawa, S., Pignatelli, M., Roy, D. S., \& Ryan, T. J. (2015). Memory engram storage and retrieval. Current Opinion in Neurobiology, 35, 101-109. https://doi.org/10.1016/j.conb.2015.07.009

Tulving, E., \& Arbuckle, T. Y. (1963). Sources of intratrial interference in immediate recall of paired associates. Journal of Verbal Learning and Verbal Behavior, 1(5), 321-334. https://doi.org/http://dx.doi.org/10.1016/S0022-5371(63)80012-2

Vaz, A. P., Yaffe, R. B., Wittig, J. H., Inati, S. K., \& Zaghloul, K. A. (2017). Dual origins of measured phase-amplitude coupling reveal distinct neural mechanisms underlying episodic memory in the human cortex. NeuroImage, 148(January), 148-159. https://doi.org/http://dx.doi.org/10.1101/084194

Veniero, D., Vossen, A., Gross, J., \& Thut, G. (2015). Lasting EEG/MEG Aftereffects of 
Rhythmic Transcranial Brain Stimulation: Level of Control Over Oscillatory Network Activity. Frontiers in Cellular Neuroscience, 9(December), 1-17.

https://doi.org/10.3389/fncel.2015.00477

Wang, X.-J. (2010). Neurophysiological and computational principles of cortical rhythms in cognition. Physiological Reviews, 90(3), 1195-268.

https://doi.org/10.1152/physrev.00035.2008

Womelsdorf, T., Schoffelen, J.-M., Oostenveld, R., Singer, W., Desimone, R., Engel, A. K., \& Fries, P. (2007). Modulation of neuronal interactions through neuronal synchronization. Science (New York, N.Y.), 316(5831), 1609-1612. https://doi.org/10.1126/science.1139597

Woods, A. J., Antal, A., Bikson, M., Boggio, P. S., Brunoni, A. R., Celnik, P., ... Nitsche, M. A. (2016). A technical guide to tDCS, and related non-invasive brain stimulation tools. Clinical Neurophysiology, 127(2), 1031-1048. https://doi.org/10.1016/j.clinph.2015.11.012

Zaehle, T., Sandmann, P., Thorne, J. D., Jäncke, L., \& Herrmann, C. S. (2011). Transcranial direct current stimulation of the prefrontal cortex modulates working memory performance: combined behavioural and electrophysiological evidence. BMC Neuroscience, 12(1), 2. https://doi.org/10.1186/1471-2202-12-2

Zwissler, B., Sperber, C., Aigeldinger, S., Schindler, S., Kissler, J., \& Plewnia, C. (2014). Shaping Memory Accuracy by Left Prefrontal Transcranial Direct Current Stimulation. The Journal of Neuroscience, 34(11), 4022 LP-4026. Retrieved from http://www.jneurosci.org/content/34/11/4022.abstract 


\section{Contributions}

For all the articles and manuscript contained in this thesis, I designed, recruited participants and collected data. I also entirely analyzed the data, plotted all the results, created all the pictures and wrote the first draft of the manuscripts.

In the first article, Philipp Knechtges recruited participants, collected data and revised the manuscript. Ivan Alekseichuk contributed running the computational model. Walter Paulus codesigned, supervised and reviewed all manuscript drafts. Andrea Antal, co-designed, supervised the study, co-wrote the introduction and reviewed all drafts.

In the second article, Ivan Alekseichuk and Zsolt Turi co-designed the study, contributed to the manuscript writing and reviewed all the drafts. Ivan Alekseichuk contributed running the computational model. Albert Lehr collected data and reviewed all drafts. Andrea Antal supervised the study and reviewed the manuscript. Walter Paulus supervised the study and co-wrote the manuscript. 


\section{Acknowledgments}

Firstly, I would like to thank my supervisor, Prof. Walter Paulus, to concede me the opportunity of pursuing my $\mathrm{PhD}$ in the department of clinical neurophysiology. This thesis wouldn't be possible without his support, comprehension and excitement about advances in basic and applied neurosciences. I also would want to warmly thank Prof. Andrea Antal for her supervision and partnership throughout my years in the department. Her contribution and support was a key factor for the history that this thesis tell. I would also like to immensely thank Prof. Michael Nitsche, for bringing me to the department and introducing me to non-invasive brain techniques. An enthusiastic appreciation to Prof. Lars Penke, my second thesis advisor, for accompanying this work's advance and helping to guide it.

I would like to thank Zsolt Turi, Ivan Alekseichuk and Elina Zmeykina for the long discussions on science and on scientific methods. I also thank and acknowledge Albert Lehr, Phillip Knechtges and Benedikt Korres for their contribution to the studies. In addition, I am grateful to Manuel Hewitt, Aguida Foerster, Aleksandra Korzeczek, Annika Primaßin, Cristina Stier, Faizal Zulkifly, Alexander Whillier, Patrik Kunz and Asif Jamil for sharing the everyday life moments that made the $\mathrm{PhD}$ years so special to me. I greatly thank Giorgi Batzikadze and Yuichiro Shirota for introducing me to TMS and discussing the methodological aspects of it. I want also to acknowledge and thank Manuel Hewitt for the technical assistance with the biomedical devices and hardware.

I also would like to thank Prof. Martin Sommer for being supportive all these years, Drs. Caspar Stephani, Dirk Czesnik and Sebastian Schade for providing medical examination for my participants. A warm thanks goes to Stephanie Pabel, for assisting in the recruitment and 
examination of participants for my projects. I am also greatly thankful to Marion Kurze for all the assistance in the bureaucratic matters along my $\mathrm{PhD}$ years.

I would like to thank all my friends in Brazil and in Göttingen, for making me what I am today and being by my side at all moments. You make life much better.

A special love and gratitude goes to my wife, Taciana, for standing by me, supporting, caring, and sharing the beautiful and harsh landscape of being alive. For all of her contributions to make me wish to be a better person every day. For giving me the gift of Dante and Otto.

Lastly, I send my love and gratitude to my parents, Antonio Alberto de Lara Junior and Anelise Amador de Lara, for making me the man that I am today. For all the effort and sacrifices, and for all the value that they give to education and to empathy. My love and thanks also go to my siblings, Mariana and Diego, and to all my family that supported and loved me for who I am. 


\section{Curriculum Vitae}

\section{Gabriel Amador de Lara}

Date of Birth: 30.08 .1983

Place of Birth: Tubarão, SC, Brazil

E-mail: gabrieldelara2@hotmail.com

\section{Education}

2015 - 2018: PhD Candidate, Neuroscience - Department of Clinical Neurophysiology, GeorgAugust University of Göttingen, Germany.

Supervisor: Prof. Dr. Walter Paulus

2010 - 2012: MSc. in Psychology - Sinos Valley University (UNISINOS), Brazil.

Supervisor: Prof. Dr. Janine Kieling Monteiro

2004 - 2008: BSc. in Psychology - Federal University of Santa Catarina (UFSC), Brazil.

\section{Work}

11/2014 - present: Department of Clinical Neurophysiology, Medical Center, Georg-August University of Göttingen, Germany. - Research Assistant, PhD candidate in Neuroscience

2010- 2013: City of Brusque (Brazil) Public Mental Health System - Coordinator and Psychologist Psychosocial Attention Center (CAPS); Community Mental Health Institution: Clinical Psychologist, 2010-2013.

Psychosocial Attention Center for Addictions (CAPSad); Addictions Treatment Institution: Coordinator and Psychologist, 2012-2013.

2011-2012: SUS Technical School (ETSUS, Blumenau), Lecturer. 


\section{Publications}

Lara, G. A. de, Alekseichuk, I., Turi, Z., Lehr, A., Antal, A., \& Paulus, W. (2017). Perturbation of theta-gamma coupling at the temporal lobe hinders verbal declarative memory. Brain Stimulation, 1-9. https://doi.org/10.1016/j.brs.2017.12.007

Lara, G. A. de, Knechtges, P. N., Paulus, W., \& Antal, A. (2017). Anodal tDCS Over the Left DLPFC Did Not Affect the Encoding and Retrieval of Verbal Declarative Information. Frontiers in Neuroscience, 11(August), 1-9. https://doi.org/10.3389/fnins.2017.00452

Alekseichuk, I., Turi, Z., Amador de Lara, G., Antal, A., \& Paulus, W. (2016). Spatial Working Memory in Humans Depends on Theta and High Gamma Synchronization in the Prefrontal Cortex. Current Biology, 26(12), 1513-1521. https://doi.org/10.1016/j.cub.2016.04.035

Lara, G. A. de, \& Monteiro, J. K. (2014). Reabilitação psicossocial de pessoas com transtornos psicóticos : atuação dos psicólogos nos CAPS de Santa Catarina. Contextos Clínicos, 7(1), 49-61. https://doi.org/10.4013/ctc.2014.71.05

Lara, G. A. de, \& Monteiro, J. K. (2012). Os psicólogos na atenção às psicoses nos CAPS. Arquivos Brasileiros de Psicologia, 76-93. http://seer.psicologia.ufrj.br/index.php/abp/article/view/776

\section{Conferences Proceedings}

Lara, G. A.; Alekseichuk, Ivan; Turi, Zsolt; Lehr, A.; Antal, Andrea; Paulus, Walter. Phasespecific theta-gamma coupling at the temporal lobe disrupts episodic memory formation (2017). 13th International Conference for Cognitive Neuroscience.

Lara, G. A.; Alekseichuk, Ivan; Turi, Zsolt; Antal, Andrea; Paulus, Walter. Affecting episodic long-term memory with tACS. In: 6th International conference on transcranial brain stimulation, (2016), Göttingen. Clinical Neurophysiology (Journal), 2016.

Lara, G. A.; Monteiro, J.K. . A atuação dos psicólogos na reabilitação psicossocial das psicoses nos CAPS de Santa Catarina. In: II Congresso Internacional de Saúde Mental e Reabilitação Psicossocial, 2012, Porto Alegre. Anais, 2012.

Lara, G. A.; Monteiro, J.K. . A atuação dos psicólogos no CAPS brasileiros - uma revisão de literatura. In: I Congresso Catarinense Psicologia Ciência e Profissão, 2011, Florianópolis. Anais do I Congresso Catarinense Psicologia Ciência e Profissão, 2011. 


\section{Scholarships}

2010-2012: CAPES scholarship covering MSc. tuitions.

\section{Idioms}

Portuguese - Fluent (mother language)
English - Fluent
Spanish - Fluent
German - Fluent 